\title{
Complete reducibility of systems of equations with respect to $\mathrm{R}$
}

\author{
Jorge Almeida* José Carlos Costa ${ }^{\dagger} \quad$ Marc Zeitoun ${ }^{\ddagger}$
}

March 21, 2007

\begin{abstract}
It is shown that the pseudovariety $\mathrm{R}$ of all finite $\mathcal{R}$-trivial semigroups is completely reducible with respect to the canonical signature. Informally, if the variables in a finite system of equations with rational constraints may be evaluated by pseudowords so that each value belongs to the closure of the corresponding rational constraint and the system is verified in $\mathrm{R}$, then there is some such evaluation which is "regular", that is one in which, additionally, the pseudowords only involve multiplications and $\omega$-powers.
\end{abstract}

2000 Mathematics Subject Classification: 20M05 (primary); 20M07, 20M35 (secondary).

Keywords: Relatively free profinite semigroup, system of equations, implicit signature, complete tameness, complete reducibility, rational constraint, $\mathcal{R}$-trivial semigroup, labeled ordinal.

\section{Introduction}

\subsection{Framework}

Since the 1960's, the theory of finite semigroups has drawn a strong motivation from applications in computer science, namely as a natural algebraic framework for classifying combinatorial phenomena described through finite automata, rational languages, or various kinds of logical formalisms 30, 31, 40, 41, 52. A central question in problems arising from such applications is to determine effectively whether a given finite semigroup belongs

\footnotetext{
${ }^{*}$ Centro de Matemática da Universidade do Porto, Departamento de Matemática Pura, Faculdade de Ciências, Universidade do Porto, Rua do Campo Alegre, 687, 4169-007 Porto, Portugal. Email: jalmeida@fc.up.pt.

${ }^{\dagger}$ Centro de Matemática, Universidade do Minho, Campus de Gualtar, 4700-320 Braga, Portugal. Email: jcosta@math.uminho.pt.

${ }^{\ddagger}$ LaBRI, Université Bordeaux 1 - CNRS UMR 5800. 351 cours de la Libération, 33405 Talence Cedex, France. Email: mz@labri.fr.
} 
to a fixed pseudovariety (that is a class closed under taking homomorphic images, subsemigroups, and finite direct products) or to show that there is no algorithm that solves the membership problem for the pseudovariety.

Pseudovarieties are often defined by describing a set of generators, which are usually constructed by applying some natural algebraic construction to members of given pseudovarieties. Thus, for two given pseudovarieties $U$ and $\mathrm{V}$, the central problem translates into solving the membership problem for their join $\mathrm{U} \vee \mathrm{V}$, their semidirect or wreath product $\mathrm{U} * \mathrm{~V}$, their bilateral semidirect or block product $\mathrm{U} \square \mathrm{V}$, their Mal'cev product $\mathrm{U}(m \mathrm{~V}$, or the power $\mathcal{P} \vee$. While there is no hope for a universal solution of this type of problem, as it has been shown that none of these operators preserves decidability of the membership problem 11, 47, 48, 23, in many particular cases the problem can be solved by exploring special structural features of the semigroups involved.

An approach which has emerged from the work of several researchers consists in finding stronger properties on the pseudovarieties upon which the operators are to be applied that will ensure that the resulting pseudovarieties will have decidable membership problem [6, 49]. This has been achieved with various degrees of generality for different operators. Ideally, the properties would be stronger than decidability of the membership problem and they would be preserved under the operators. At present no (reasonable) such properties have been established.

The key property which intervenes in the partially successful approach has been formulated by Steinberg and the first author [16, 15, following earlier attempts by the first author 3 and extending seminal work by Ash 22 . Basically, Ash's theorem establishes the key property for the pseudovariety of all finite groups, thereby proving the type II and pointlike conjectures of Rhodes 32. The key property has been called inevitability by Ash, for whom it appeared in the special case of the pseudovariety of all finite groups under a very particular form which is not readily extendible to other pseudovarieties, and reducibility by Steinberg and the first author (see [6] and Section 3 for further details).

\subsection{Reducibility}

The definition of reducibility is motivated by two observations. First, a pseudovariety defined from pseudovarieties with decidable membership by applying computable operators (which is the common situation) is recursively enumerable, so there already exists a semi-algorithm for testing membership. Second, one can obtain in many cases a description of such a pseudovariety in terms of pseudoidentitie 1 A typical situation in which

\footnotetext{
${ }^{1}$ Recall that a pseudoword is an element of an $A$-generated free profinite semigroup $\bar{\Omega}_{A} \mathrm{~S}$, where $A$ is a finite alphabet. A pseudoidentity $(u, v)$ is a pair of pseudowords, noted $u=v$. A semigroup $S$ satisfies the pseudoidentity $u=v$, written $S \models u=v$, if
} 
such a description can be achieved occurs in the computation of Mal'cev products: a pseudoidentity basis for $\mathrm{U} m \mathrm{~V}$ is obtained by substituting, in each pseudoidentity of a basis $\Sigma$ of $U$, all letters by pseudowords evaluating on $\mathrm{V}$ to the same idempotent [42]. Thus, a semi-algorithm for non-membership of a finite semigroup $S$ to $\mathrm{U} \oplus \mathrm{V}$ amounts to finding a pseudoidentity $u\left(z_{1}, \ldots, z_{n}\right)=v\left(z_{1}, \ldots, z_{n}\right)$ of $\Sigma$ on alphabet $\left\{z_{1}, \ldots z_{n}\right\}$ and pseudowords $w_{1}, \ldots, w_{n}$ evaluating to the same idempotent on $\mathrm{V}$, such that $S$ fails $u\left(w_{1}, \ldots, w_{n}\right)=v\left(w_{1}, \ldots, w_{n}\right)$. This is equivalent to finding $s_{1}, \ldots, s_{n} \in S$ with $u\left(s_{1}, \ldots, s_{n}\right) \neq v\left(s_{1}, \ldots, s_{n}\right)$ and a continuous morphism $\varphi: \bar{\Omega}_{A} S \rightarrow S$, such that there exist $w_{1}, \ldots, w_{n} \in \bar{\Omega}_{A} S$ satisfying

$$
\begin{aligned}
& \mathrm{V} \models w_{1}^{2}=w_{1}=w_{2}=\cdots=w_{n}, \text { and } \\
& \varphi\left(w_{i}\right)=s_{i} \text { for } i=1, \ldots, n .
\end{aligned}
$$

Let $X=\left\{x_{1}, \ldots, x_{n}\right\}$ be a set of variables. In the present situation, the function mapping $x_{i}$ to $w_{i}$ is called a solution modulo $\mathrm{V}$ of the system

$$
x_{1}^{2}=x_{1}=x_{2}=\cdots=x_{n}
$$

with constraints $\left(s_{1}, \ldots, s_{n}\right) \in S^{n}$ associated to $\left(x_{1}, \ldots, x_{n}\right)$.

Since $\mathrm{U}$ is decidable, one can enumerate all tuples $\left(s_{1}, \ldots, s_{n}\right)$ of $S$ failing a given pseudoidentity of $\mathrm{U}$. Therefore, deciding membership in $\mathrm{U}(\mathrm{m}) \mathrm{V}$ is reduced to obtaining a semi-algorithm for solving a system (1.1) of word equations modulo the pseudovariety $\mathrm{V}$, where solutions are pseudowords constrained, by (1.2), to be chosen from the given clopen sets $\varphi^{-1}\left(s_{i}\right)$, that is from the topological closure of given rational languages of $A^{+}$[2, Section 3.6].

Now, the universe of possible solutions is far too large, namely uncountable provided $A \neq \emptyset$, to permit an algorithmic treatment. Informally, the pseudovariety $\mathrm{V}$ is said to be reducible if every such system which has a solution also admits a solution using pseudowords of a restricted kind. To define the restricted pseudowords, one fixes a set of pseudowords $\sigma$, called an implicit signature. Elements of $\sigma$ can be naturally viewed as (implicit) operations under substitution, and one takes those pseudowords which can be generated from the letters of $A$ by applying these operations. We say that $\mathrm{V}$ is $\sigma$-reducible for a class of equation systems if the existence of a solution modulo $\mathrm{V}$ of any system in the class entails the existence of a solution in $\sigma$-terms. The signature $\sigma$ should also possess reasonable algorithmic properties, in order for the $\sigma$-reducibility of a pseudovariety to imply decidability of its membership problem:

(1) as a set, $\sigma$ should be recursively enumerable;

$\varphi(u)=\varphi(v)$ for any continuous morphism $\varphi: \bar{\Omega}_{A} \mathrm{~S} \rightarrow S$. Given a set $\Sigma$ of pseudoidentities, the class $\llbracket \Sigma \rrbracket$ of all finite semigroups satisfying all pseudoidentities of $\Sigma$ is a pseudovariety. Conversely, any pseudovariety is of the form $\llbracket \Sigma \rrbracket$, and one says that it is defined by the pseudoidentity basis $\Sigma$ [4]. See [2] and Section 3$]$ 
(2) as implicit operations, the elements of $\sigma$ should be effectively computable in finite semigroups;

(3) the word problem for the free $\sigma$-algebra in the variety generated by $\mathrm{V}$ should be decidable.

Properties (1) and (2) mean that $\sigma$ forms a countable set $\left\{\pi_{0}, \pi_{1}, \ldots\right\}$, and that there exists a Turing machine outputting a sequence of Turing machines $M_{0}, M_{1}, \ldots$, where $M_{i}$ computes the implicit operation $\pi_{i}$. That is, given a semigroup $S$ and elements $s_{1}, \ldots, s_{k_{i}}$ of $S$, where $k_{i}$ is the arity of $\pi_{i}$, the machine $M_{i}$ outputs $\pi_{i}\left(s_{1}, \ldots, s_{k_{i}}\right)$. From hereon, we only consider signatures satisfying the algorithmic properties (1) (3)

These properties, combined with the narrowing of the set where solutions can be searched for (that is, $\sigma$-reducibility), yield a semi-algorithm for solving systems modulo $\mathrm{V}$ with constraints: by (1)] one can enumerate tuples $w_{1}, \ldots, w_{n}$ of elements of the free $\sigma$-algebra as candidates for a solution; by (2) one can evaluate them in finite semigroups so that (1.2) can be checked; finally by (3)] one can test whether a candidate tuple satisfies an equation system, such as (1.1).

The restriction on the form of the equation systems is motivated by decidability results we want to obtain. For instance, systems of the form $x_{1}^{2}=x_{1}=x_{2}=\cdots=x_{n}$ are those that are required to obtain decidability results for Mal'cev products. For some semidirect products, the Basis Theorem [20] $]^{2}$ leads to the same reduction. In this case, the systems are associated with finite directed graphs as follows: the variables are the vertices and the edges of the graph; for each edge $v \stackrel{e}{\rightarrow} w$, the system includes the equation $v e=w$. Initially, it was this latter type of system that was considered in [16], where a pseudovariety $\mathrm{V}$ is called $\sigma$-tame if it is $\sigma$-reducible for such equation systems, and satisfies (1) (3) These systems are one-sided: the variables $y$ in equations $x y=z$ can only appear once in the whole system, whereas the variables $x$ and $z$ may appear several times and in either position. This means in particular that proving that $\mathrm{V}$ is tame does not in general entail that the dual pseudovariety is also tame. To overcome this difficulty and to treat globally a more general situation, the notion of complete $\sigma$-tameness (also called complete $\sigma$-reducibility in this paper, under the assumption of (1) (3) was introduced in 6 to refer to a pseudovariety which is $\sigma$-reducible for all finite systems of pseudoword equations in which the pseudowords that define the equations are given by $\sigma$-terms. Unlike tameness, this property is inherited by the dual pseudovariety.

\footnotetext{
${ }^{2}$ The proof of the Basis Theorem is known to have a gap in its full generality. However, the statement holds in some relevant cases. Whether it is valid in full generality remains an open problem. See [6, 53 49] for further information.
} 


\subsection{Known results}

The implicit signature most often consists of just two pseudowords: $a b$, representing semigroup multiplication, and $a^{\omega-1}$, the unary pseudoinverse which, evaluated on an element $s$ of a profinite semigroup, takes the value of the unique inverse of $s e$ in the maximal subgroup of the closed subsemigroup generated by $s$, where $e$ denotes the idempotent of that subgroup. Following [15, we will say that this signature is canonical, and denote it $\kappa$.

There are several examples of reducibility results in the literature. In the following incomplete sample, if no mention to the contrary is made, the implicit signature is canonical. The pseudovariety $\mathrm{G}$ of all finite groups is tame by the theorem of Ash 22 mentioned above together with a little extra work 3, 15. To justify the interest of the notion of reducibility, it is also worth mentioning that the reducibility of $G$ has been rediscovered in disguise in Model Theory [33, 12, 13]. On the other hand, G is not completely $\kappa$-tame by [29]. In contrast, the pseudovariety $\mathrm{G}_{p}$ of all finite $p$ groups is not tame [15] for the canonical signature, basically because the free group is residually a finite $p$-group for every prime $p$ [25]. However, using results of several authors [50, 38, 51] and analogies with Symbolic Dynamics, the first author has exhibited an infinite implicit signature with respect to which $\mathrm{G}_{p}$ is tame [5]. The pseudovariety $A b$ of all finite Abelian groups is completely tame [14. The pseudovariety LSI of all finite local semilattices is tame 28. The pseudovariety $\mathrm{R}$ of all finite semigroups in which the Green relation $\mathcal{R}$ is trivial is tame, which entails the reducibility of several associated pseudovarieties such as R $\vee$ G 11. Earlier attempts to extend the reducibility of $R$ to more general systems of equations, with the aim of proving that the dual pseudovariety $\mathrm{L}$, of all finite $\mathcal{L}$-trivial semigroups, is also reducible have only succeeded in some very special cases [4].

\subsection{Contributions}

The present paper explores the methods of Makanin [36, 37] (see also [35. Chapter 12]) and a good knowledge about the structure of free pro- $\mathrm{R}$ semigroups [19, 21] and particularly of the free $\kappa$-algebras in the variety generated by $\mathrm{R}[21$, to prove that $\mathrm{R}$ is completely reducible. The strong analogy between Makanin's algorithm to solve, in free semigroups, systems of word equations, also with rational constraints on the variables, and our reducibility problem in general stems from the fact that the algorithm consists in a skillful combinatorial analysis to reduce the existence of an arbitrary solution of a given system to the existence of a solution of a special kind, namely one whose exponents of periodicity are kept under control.

The basic difficulty in dealing with word equations, also when pseudowords are involved, is the propagation of factorizations resulting from the appearance of the same variable in different places. This problem is suitably 
handled by Makanin's algorithm when solutions in words are sought, computable bounds being established for how far the refinement process needs to be performed. The difficulty is in a sense of the same nature when a similar method is applied for a pseudovariety $\mathrm{V}$ such that, for two factorizations of the same pseudoword modulo $\mathrm{V}$, there is a notion of common refinement. This is the case for R, but also for many pseudovarieties, and so, it should be possible to extend the methods developed in this paper to establish complete reducibility in other cases. It is with such extensions in mind that we formulate in Section 3 a number of simplifications of the problem for general pseudovarietie 3 .

A key difficulty is that it may be necessary to continue the refinement process of factorizations indefinitely and even infinitely often (which of course is not the case for finite words), but it will hopefully be possible to take advantage of regularity patterns to obtain the desired reducibility result. This program is carried out successfully in this paper for the pseudovariety R.

The paper is organized as follows. We set up the notation in Section 2 We show in Section 3 that for a broad class of pseudovarieties including R, proving reducibility for general equation systems (with variables as well as parameters) can be reduced to solving the problem for systems consisting in a single equation without parameters. The structure of pseudowords of $\bar{\Omega}_{A} \mathrm{R}$, which can be seen as particular labeled ordinals, is reviewed in Sections 4 and 5 and exploited in Section 6 to further simplify the equation systems. In Section [7, we formulate the problem in terms of systems of boundary equations. The main tools to manipulate these systems are presented in Section 8 . Finally, the problem formulated in Section 7 for systems of boundary equations is solved in Section 9 .

\section{Definitions and notation}

Given a semigroup $S$, we let $\leq_{\mathcal{R}} \subseteq S \times S$ be the relation defined by $s \leq_{\mathcal{R}} t$ if and only if $s S^{1} \subseteq t S^{1}$. The Green relation $\mathcal{R}$ is defined by $s \mathcal{R} t$ if and only if $s \leq_{\mathcal{R}} t$ and $t \leq_{\mathcal{R}} s$. We write $s<_{\mathcal{R}} t$ if $s \leq_{\mathcal{R}} t$, and $s$ and $t$ are not $\mathcal{R}$-equivalent. A semigroup is $\mathcal{R}$-trivial if $s \mathcal{R} t$ implies $s=t$. We denote by $\mathrm{R}$ the pseudovariety of all $\mathcal{R}$-trivial semigroups.

A finite semigroup is viewed as a topological semigroup under the discrete topology. Recall that a profinite semigroup is a compact residually finite semigroup. More generally, if $\mathrm{V}$ is a pseudovariety of semigroups, then a pro-V semigroup is a compact semigroup which is residually in $\mathrm{V}$. The free

\footnotetext{
${ }^{3}$ To solve word equations, there is a more efficient (PSPACE) algorithm than Makanin's, due to Plandowski [44, 45. We are concerned here with an abstract property rather than the construction of an algorithm, and there is no complexity issue in this paper. This justifies the use of Makanin's ideas rather than Plandowski's, since the former seem to be more adjusted to the current problem.
} 
pro-V semigroup on a free finite set of generators $A$ (also called an alphabet) may be constructed as the completion $\bar{\Omega}_{A} \mathrm{~V}$ of the free semigroup $A^{+}$with respect to the (pseudo) ultrametric defined by $d_{\mathrm{V}}(u, v)=2^{-r_{\mathrm{V}}(u, v)}$, where $r_{\mathrm{V}}(u, v)$ is the smallest cardinality of a semigroup $S \in \mathrm{V}$ such that $S$ fails the identity $u=v$, in case such a semigroup exists, and $d \vee(u, v)=0$ otherwise. See [8] for further details.

In particular, it is important to note that indeed $\bar{\Omega}_{A} \mathrm{~V}$ has the universal property which justifies calling it a free pro- $\mathrm{V}$ semigroup on the set $A$ of free generators: given any function $\varphi: A \rightarrow S$ into a pro-V semigroup $S$, there is a unique continuous homomorphism $\hat{\varphi}: \bar{\Omega}_{A} \mathrm{~V} \rightarrow S$ such that $\hat{\varphi} \circ \iota=\varphi$, where $\iota: A \rightarrow \bar{\Omega}_{A} \mathrm{~V}$ is the natural mapping (that associates with each letter $a \in A$ the equivalence class of the constant sequence with value $a$ ). The mapping $\hat{\varphi}$ is also said to be induced by $\varphi$. It follows that, if $A$ is a subset of the finite set $B$, then the unique continuous homomorphism $\bar{\Omega}_{A} \mathrm{~V} \rightarrow \bar{\Omega}_{B} \mathrm{~V}$ induced by the inclusion mapping $A \hookrightarrow B$ is injective. We will identify $\bar{\Omega}_{A} \vee$ with its image and therefore view $\bar{\Omega}_{A} \mathrm{~V}$ as the closed subsemigroup of $\bar{\Omega}_{B} \mathrm{~V}$ generated by $A$.

Let $\mathrm{S}$ be the pseudovariety of all finite semigroups. The elements of $\left(\bar{\Omega}_{A} S\right)^{1}$, over arbitrary finite alphabets $A$, will be called pseudowords. They may also be viewed as $A$-ary implicit operations on profinite semigroups as follows: given a profinite semigroup $S$, a pseudoword $w \in \bar{\Omega}_{A} \mathrm{~S}$, and an evaluation mapping $\varphi: A \rightarrow S$, since $\bar{\Omega}_{A} \mathrm{~S}$ is freely generated by $A$ as a profinite semigroup, there is a unique extension of $\varphi$ to a continuous homomorphism $\hat{\varphi}: \bar{\Omega}_{A} S \rightarrow S$. We let $w_{S}(\varphi)=\hat{\varphi}(w)$. This interpretation of $w$ as an operation, which is also called the natural interpretation, commutes with continuous homomorphisms between profinite semigroups. In particular, if $S=2^{A}$ is the semigroup of all subsets of $A$ under the operation of binary union and $\varphi: A \rightarrow S$ sends each letter $a$ to $\{a\}$, then $w \in \bar{\Omega}_{A} \mathrm{~S} \mapsto w_{S}(\varphi) \in 2^{A}$ is a continuous homomorphism which is denoted by $c$ and which is known as the content function. A letter $a$ is said to occur in the pseudoword $w$ if $a \in c(w)$.

A pseudoidentity is a formal equality of the form $u=v$ with $u, v \in \bar{\Omega}_{A} \mathrm{~S}$ for some finite alphabet $A$. We say that a profinite semigroup $S$ satisfies $u=v$ and we write $S \models u=v$ if $u_{S}=v_{S}$. A class $\mathcal{C}$ of profinite semigroups satisfies a set $\Sigma$ of pseudoidentities, noted $\mathcal{C} \models \Sigma$, if every semigroup of $\mathcal{C}$ satisfies every pseudoidentity of $\Sigma$. Note that if $c(u) \cup c(v) \subseteq A$, then $\bar{\Omega}_{A} \bigvee \models$ $u=v$ if and only if every pro- $\mathrm{V}$ semigroup satisfies the pseudoidentity $u=v$, that is, if and only if $\mathrm{V} \models u=v$, in which case we will sometimes also write $u=\mathrm{v} v$.

We start with the formal setup of the problem of reducibility of a system of equations for a general pseudovariety $\mathrm{V}$ of semigroups. Let $X$ be a finite set, whose elements will be the variables of the system which are to be evaluated in order to solve the system. We also consider a finite set $P$ of parameters, disjoint from $X$, whose evaluation is fixed from the start. In 
word equations with rational constraints on the variables, there is no need for parameters as the evaluation of a variable can be fixed by stipulating a singleton rational constraint. This gimmick does not work in the setting of a free profinite semigroup with clopen constraints since the only clopen singletons are those consisting of finite words. Yet we show in Proposition 3.1 below that parameters may be systematically avoided in some circumstances.

We consider a finite system of pseudoword equations

$$
u_{i}=v_{i} \quad(i=1, \ldots, r)
$$

where each of the pseudowords $u_{i}$ and $v_{i}$ belongs to the free profinite semigroup $\bar{\Omega}_{X \cup P} S$. We also fix a finite set $A$ which is the free generating set of the free profinite semigroup $\bar{\Omega}_{A} \mathrm{~S}$ in which "solutions modulo V" are sought. To each $x \in X$ we associate a constraint which is a clopen subset $K_{x}$ of $\bar{\Omega}_{A} \mathrm{~S}$. To each parameter $p \in P$ we associate an element $\pi_{p} \in \bar{\Omega}_{A} \mathrm{~S}$. A solution of the system (2.1) modulo $\mathrm{V}$ satisfying the constraints is given by a continuous homomorphism $\delta: \bar{\Omega}_{X \cup P} S \rightarrow \bar{\Omega}_{A} S$ such that the following conditions are satisfied:

(1) $\delta(x) \in K_{x}$ for every $x \in X$;

(2) $\delta(p)=\pi_{p}$ for every $p \in P$;

(3) the pseudovariety $\mathrm{V}$ satisfies each of the pseudoidentities $\delta\left(u_{i}\right)=\delta\left(v_{i}\right)$ $(i=1, \ldots, r)$.

Let $\sigma$ be an implicit signature, by which we mean a set of pseudowords including the pseudoword $a b \in \bar{\Omega}_{\{a, b\}} \mathrm{S}$. Under the natural interpretation of the elements of $\sigma$, every profinite semigroup may be viewed as a $\sigma$-algebra in the sense of Universal Algebra [27]. The $\sigma$-subalgebra of $\bar{\Omega}_{A} \mathrm{~S}$ generated by $A$ is denoted $\Omega_{A}^{\sigma} S$ and can be easily shown to be freely generated by $A$ in the variety of $\sigma$-algebras generated by the pseudovariety $\mathrm{S}$ of all finite semigroups. The most encountered example of such a signature is $\left\{a b, a^{\omega-1}\right\}$, where the implicit operation $a^{\omega-1}$ is defined in Section 1

Consider a system of the form (2.1), with clopen constraints $K_{x} \subseteq \bar{\Omega}_{A} \mathrm{~S}$ $(x \in X)$, with the restrictions that $u_{i}, v_{i} \in \Omega_{X \cup P}^{\sigma} \mathrm{S}$ and for every parameter $p \in P, \pi_{p} \in \Omega_{A}^{\sigma} \mathrm{S}$. Assume that this system has a solution modulo $\mathrm{V}$. The pseudovariety $\mathrm{V}$ is said to be $\sigma$-reducible for this system if it also has a solution $\delta: \bar{\Omega}_{X \cup P} \mathrm{~S} \rightarrow \bar{\Omega}_{A} \mathrm{~S}$ modulo $\mathrm{V}$ with the additional property $\delta(X) \subseteq$ $\Omega_{A}^{\sigma} \mathrm{S}$. We call such a function $\delta$ a solution modulo $\mathrm{V}$ in $\sigma$-terms. We say that $\mathrm{V}$ is completely $\sigma$-reducible if it is $\sigma$-reducible for every such system.

\section{Preliminary simplifications}

Let us proceed with the simplifications which are the objective of this section. The first one consists in a simple observation stating that, just as in the 
word case, we may consider only systems without parameters, even though parameters cannot be directly captured by the constraints as in the word case.

Proposition 3.1 Let $\mathrm{V}$ be an arbitrary pseudovariety. If $\mathrm{V}$ is $\sigma$-reducible for systems of equations of $\sigma$-terms without parameters, then $\mathrm{V}$ is completely $\sigma$-reducible.

Proof. Consider a system of the form (2.1) with $u_{i}, v_{i} \in \Omega_{X \cup P}^{\sigma} \mathrm{S}$, clopen constraints $K_{x} \subseteq \bar{\Omega}_{A} \mathrm{~S}(x \in X)$, and parameter values $\pi_{p} \in \Omega_{A}^{\sigma} \mathrm{S}(p \in P)$. We consider an enlarged set of variables $Y=X \uplus A$ together with an empty set of parameters. The constraints for the elements of $X$ are kept as given while, for each new variable $a \in A$, the corresponding constraint is the singleton set $K_{a}=\{a\}$ (which is clopen in $\bar{\Omega}_{A} \mathrm{~S}$ ). We also modify the equations as follows. We consider the unique continuous homomorphism $\psi: \bar{\Omega}_{X \cup P} \mathrm{~S} \rightarrow \bar{\Omega}_{Y} \mathrm{~S}$ mapping each element of $X$ to itself and each $p \in P$ to $\pi_{p}$, which can now be viewed as an element of $\bar{\Omega}_{Y} S$ (since $A \subseteq Y$ ) as argued in Section 2. Note that, since $\pi_{p} \in \Omega_{A}^{\sigma} \mathrm{S}$ for all $p \in P, \psi$ maps each $u \in \Omega_{X \cup P}^{\sigma} \mathrm{S}$ to $\psi(u) \in \Omega_{Y}^{\sigma} \mathrm{S}$ : since $\psi$ simply replaces some letters in $\sigma$-terms by $\sigma$-terms, it maps a $\sigma$-term to a $\sigma$-term. We consider the new system

$$
\psi\left(u_{i}\right)=\psi\left(v_{i}\right) \quad(i=1, \ldots, r)
$$

over the set of variables $Y$ under the constraints $K_{y}(y \in Y)$.

Suppose that $\delta: \bar{\Omega}_{X \cup P} S \rightarrow \bar{\Omega}_{A} S$ is a solution modulo $V$ of the system (2.1) under the original constraints and parameter values. Then $\delta(p)=$ $\pi_{p}$ for every $p \in P$ so that the unique continuous homomorphism $\delta^{\prime}: \bar{\Omega}_{Y} \mathrm{~S} \rightarrow$ $\bar{\Omega}_{A} \mathrm{~S}$ which is the identity on $A$ and coincides with $\delta$ on $X$ is such that $\delta^{\prime} \circ \psi=\delta$ and so $\delta^{\prime}$ is a solution modulo $\mathrm{V}$ of the new system (3.1). Conversely, assume that $\varepsilon^{\prime}: \bar{\Omega}_{Y} \mathrm{~S} \rightarrow \bar{\Omega}_{A} \mathrm{~S}$ is a solution modulo $\mathrm{V}$ of the system (3.1) under the constraints $K_{y}(y \in Y)$. It is then a routine matter to check that $\varepsilon=\varepsilon^{\prime} \circ \psi$ is a solution modulo $\mathrm{V}$ of the original system (2.1). For instance, to verify that $\varepsilon(p)=\pi_{p}$ for every $p \in P$, since $\varepsilon(p)=\varepsilon^{\prime}(\psi(p))=\varepsilon^{\prime}\left(\pi_{p}\right)$, it suffices to observe that $\varepsilon^{\prime}\left(\pi_{p}\right)=\pi_{p}$ because $\varepsilon^{\prime}(a) \in K_{a}=\{a\}$ for every $a \in A$ and $\pi_{p} \in \Omega_{A}^{\sigma} S \subseteq \bar{\Omega}_{A} \mathrm{~S}$.

Finally, suppose that $\delta$ is a solution modulo $\mathrm{V}$ of (2.1) and let $\delta^{\prime}$ be the solution modulo $\mathrm{V}$ of (3.1) constructed as above. By hypothesis, there is a solution $\varepsilon^{\prime}$ of (3.1) modulo $\vee$ which assigns to all variables $y \in Y$ elements of $\Omega_{A}^{\sigma} S$. By the above, $\varepsilon$ is a solution modulo $V$ of (2.1) which coincides with $\varepsilon^{\prime}$ on $X$ and so, in particular, assigns to all variables elements of $\Omega_{A}^{\sigma} \mathrm{S}$. Hence $\mathrm{V}$ is completely $\sigma$-reducible.

We will say that the pseudovariety $\mathrm{V}$ is weakly cancellable if, whenever $\mathrm{V}$ satisfies a pseudoidentity of the form $u_{1} \# u_{2}=v_{1} \# v_{2}$, where \# is a letter which does not occur in any of the pseudowords $u_{1}, u_{2}, v_{1}, v_{2}$, then $\mathrm{V}$ also satisfies the pseudoidentities $u_{1}=v_{1}$ and $u_{2}=v_{2}$. 
Of course, no nontrivial pseudovariety of commutative semigroups is weakly cancellable. Yet, there are many common examples of weakly cancellable pseudovarieties such as R [9] and J (finite $\mathcal{J}$-trivial semigroups) 2 . A stronger requirement is that $V$ be closed under Birget expansions [26] 4] which is the case for any pseudovariety of the form $\mathrm{V}=\mathrm{B} \times \mathrm{W}$ [17, where $B$ is the pseudovariety of all finite bands (semigroups in which all elements are idempotents), which in turn is equivalent to $\mathrm{V}=\mathrm{B} m \mathrm{~V}$. Using these results, one may show that pseudovarieties such as OCR (finite orthodox completely regular semigroups), CR (finite complete regular semigroups), $\overline{\mathrm{H}}$ (finite semigroups whose subgroups lie in a given pseudovariety of groups $\mathrm{H}$ ), DA (finite semigroups in which regular elements are idempotents), DO (finite semigroups in which regular $\mathcal{D}$-classes are orthodox subsemigroups), DS (finite semigroups in which regular $\mathcal{D}$-classes are subsemigroups), as well as any meet or join of some of these pseudovarieties, are weakly cancellable [17. Another type of example of weakly cancellable pseudovariety is given by any arborescent pseudovariety of groups $\mathrm{H}$ [18, which may be characterized by the property that $\mathrm{H}=(\mathrm{H} \cap \mathrm{Ab}) * \mathrm{H}$ (the name standing for the profinite Cayley graphs of its relatively profinite groups being profinite trees; see also [24]). This latter type of example includes the pseudovarieties $G$ and $\mathrm{G}_{p}$ which already appeared in Section $[1 \mathrm{i}$. Thus indeed weak cancellability is a rather common and weak property, which justifies the interest of the simplification of the reducibility problem given by the following result.

Proposition 3.2 Let $\mathrm{V}$ be a weakly cancellable pseudovariety. If $\mathrm{V}$ is $\sigma$ reducible for systems consisting of just one equation of $\sigma$-terms without parameters, then $\mathrm{V}$ is completely $\sigma$-reducible.

Proof. Consider an arbitrary system (2.1) of $r$ equations of $\sigma$-terms. By Proposition 3.1, it suffices to consider the case in which the set of parameters is empty. We consider an enlarged set of variables $Y=X \uplus\left\{\#_{1}, \ldots, \#_{r-1}\right\}$ and we also enlarge the alphabet for the solutions which becomes $B=$ $A \uplus\left\{\#_{1}, \ldots, \#_{r-1}\right\}$. We add singleton constraints $\left\{\#_{i}\right\}$ for each of the new variables $\#_{i}$ and we consider the single equation

$$
u_{1} \#_{1} \cdots u_{r-1} \#_{r-1} u_{r}=v_{1} \#_{1} \cdots v_{r-1} \#_{r-1} v_{r}
$$

under the old and new constraints over the alphabet $B$. Note that $\bar{\Omega}_{A} \mathrm{~S}$ is a clopen subset of $\bar{\Omega}_{B} \mathrm{~S}$ so that indeed the given constraints $K_{x}$ on the variables $x \in X$ may be considered as constraints over the enlarged alphabet $B$.

Assume $\delta: \bar{\Omega}_{X} \mathrm{~S} \rightarrow \bar{\Omega}_{A} \mathrm{~S}$ is a solution modulo $\mathrm{V}$ of the original system (2.1). We extend $\delta$ to the unique continuous homomorphism $\delta^{\prime}: \bar{\Omega}_{Y} S \rightarrow$

\footnotetext{
${ }^{4}$ Birget expansions are obtained by iterating alternately right and left Rhodes expansions, cutdown to generators. Some properties of right Rhodes expansions are recalled in Section 4 where they also play a role.

${ }^{5}$ See also [10] for further remarks on weakly cancellable pseudovarieties of groups.
} 
$\bar{\Omega}_{B} S$ which coincides with $\delta$ on $\bar{\Omega}_{X} S$ and which is the identity on the new variables $\#_{i}$. Then $\delta^{\prime}$ is a solution modulo $V$ of the equation (3.2). Since $\mathrm{V}$ is assumed to be $\sigma$-reducible for such equations, there is some solution $\varepsilon^{\prime}: \bar{\Omega}_{Y} S \rightarrow \bar{\Omega}_{B} S$, modulo $\mathrm{V}$, of the equation (3.2) which assigns to all variables pseudowords defined by $\sigma$-terms. We let $\varepsilon$ be the restriction of $\varepsilon^{\prime}$ to $\bar{\Omega}_{X} \mathrm{~S}$. Since $\varepsilon^{\prime}(x) \in K_{x} \subseteq \bar{\Omega}_{A} \mathrm{~S}, \varepsilon$ assumes its values in $\Omega_{A}^{\sigma} \mathrm{S}$. On the other hand, by applying $\varepsilon^{\prime}$ to both sides of the equation (3.2) we obtain the pseudoidentity

$$
\varepsilon\left(u_{1}\right) \#_{1} \cdots \varepsilon\left(u_{r-1}\right) \#_{r-1} \varepsilon\left(u_{r}\right)=\varepsilon\left(v_{1}\right) \#_{1} \cdots \varepsilon\left(v_{r-1}\right) \#_{r-1} \varepsilon\left(v_{r}\right)
$$

which is valid in $\mathrm{V}$. Since $\mathrm{V}$ is weakly cancellable, it follows that each of the pseudoidentities $\varepsilon\left(u_{i}\right)=\varepsilon\left(v_{i}\right)(i=1, \ldots, r)$ is valid in $\vee$. Hence $\varepsilon$ is a solution of the original system modulo $\mathrm{V}$. This shows that $\mathrm{V}$ is completely $\sigma$-reducible.

The constraints have been formulated in the simplest terms possible, namely using clopen subsets of the free profinite semigroup $\bar{\Omega}_{A} \mathrm{~S}$ where solutions modulo $\mathrm{V}$ are sought. It is however often more convenient to work instead with constraints determined by fixing a continuous homomorphism $\varphi: \bar{\Omega}_{A} S \rightarrow S$ into a finite semigroup $S$ and stipulating that the value $\delta(x)$ attributed to each variable $x$ should be such that $\varphi(\delta(x))=s_{x}$ is a given element of $S$. Using Hunter's Lemma [34, it is easy to see that, given any finite set of clopen subsets of a profinite semigroup $T$, there exists a continuous homomorphism $\varphi: T \rightarrow S$ into a finite semigroup $S$ such that each of the given clopen subsets is of the form $\varphi^{-1}(Q)$ for some subset $Q$ of $S$ [7]. Under these circumstances, one also says that the clopen subsets are recognized by $\varphi$. Hence clopen constraints can be split into disjoint unions of constraints given by values under continuous homomorphisms into finite semigroups. It follows that the two notions of reducibility, using clopen constraints or constraints given by continuous homomorphisms into finite semigroups, are equivalent.

By Proposition 3.2, one may assume the following simplified setup for the proof that a weakly cancellable pseudovariety $\mathrm{V}$ is completely $\kappa$-reducible.

Data The following are given:

(D.1) two finite sets $X$ and $A$;

(D.2) a single equation $u=v$, with $u, v \in \Omega_{X}^{\kappa} \mathrm{S}$;

(D.3) a continuous homomorphism $\varphi: \bar{\Omega}_{A} S \rightarrow S$ into a finite semigroup $S$ and, for each $x \in X$, an element $s_{x} \in S$ which is used to define the clopen constraint $\varphi^{-1}\left(s_{x}\right) \subseteq \bar{\Omega}_{A} \mathrm{~S}$;

(D.4) a solution modulo $\mathrm{V}$ of the equation $u=v$ satisfying the constraints, which is given by an evaluation $\delta: \bar{\Omega}_{X} \mathrm{~S} \rightarrow \bar{\Omega}_{A} \mathrm{~S}$ such that

$$
\left\{\begin{array}{l}
\mathrm{V} \models \delta(u)=\delta(v) \\
\varphi(\delta(x))=s_{x} \quad(x \in X) .
\end{array}\right.
$$


Objective To show that there exists a solution, modulo $\mathrm{V}$, using only $\kappa$ terms, of the equation $u=v$ which satisfies the constraints.

For the remainder of the paper, we will consider this setup for the specific pseudovariety $\mathrm{V}=\mathrm{R}$.

\section{Structural aspects of $\bar{\Omega}_{A} \mathrm{R}$}

Before introducing some specific tools to handle pseudowords modulo the pseudovariety $\mathrm{R}$, we recall the following finite cancellability result for pseudowords, which is stated here formally for later reference. An elementary proof can be found in [21, Proposition 2.1]. See also the discussion following Proposition 5.4 in [1].

Proposition 4.1 ([21, Proposition 2.1]) Let $x, y, z, t$ be pseudowords and let $a, b$ be letters such that $x a y=z b t$ and $a \notin c(x)$ and $b \notin c(z)$. If either $c(x)=c(z)$ or $c(x a)=c(z b)$, then $x=z, a=b$, and $y=t$.

Applying inductively Proposition 4.1 we obtain the following result, where we also include the left-right dual statement.

Corollary 4.2 Let $w$ be a pseudoword over a finite alphabet.

(1) There is a unique factorization $w=a_{1} w_{1} \cdots a_{n} w_{n}$ where, for $i=2, \ldots, n$, $a_{i} \notin c\left(a_{1} w_{1} \cdots a_{i-1} w_{i-1}\right)$ and $c(w)=\left\{a_{1}, \ldots, a_{n}\right\}$.

(2) There is a unique factorization $w=w_{n} a_{n} \cdots w_{1} a_{1}$ where, for $i=2, \ldots, n$, $a_{i} \notin c\left(w_{i-1} a_{i-1} \cdots w_{1} a_{1}\right)$ and $c(w)=\left\{a_{1}, \ldots, a_{n}\right\}$.

The factorizations of Corollary 4.2 will be called, respectively, the firstoccurrences and the last-occurrences factorizations.

For $u \in \bar{\Omega}_{A} \mathrm{~S}$, let $\vec{c}(u)$ denote the cumulative content of $u$, that is the set of all letters $a \in A$ such that $\mathrm{R} \models u a=u$. It follows immediately from this definition that for $u, v \in \bar{\Omega}_{A} \mathrm{~S}$, if R satisfies $u=v$, then $\vec{c}(u)=\vec{c}(v)$.

If, instead of the pseudoidentity $u a=u$, we consider the pseudoidentity $u v=u$ where $v$ is a pseudoword, then we have the following result.

Lemma 4.3 Let $u, v \in \bar{\Omega}_{A} \mathrm{~S}$. Then $\mathrm{R} \models u v=u$ if and only if $c(v) \subseteq \vec{c}(u)$. In particular, $\mathrm{R} \models u^{2}=u$ if and only if $c(u)=\vec{c}(u)$.

Proof. Suppose first that $c(v) \subseteq \vec{c}(u)$. Since the content function $c$ is continuous and $A^{+}$is dense in $\bar{\Omega}_{A} \mathrm{~S}$, there is a sequence $\left(w_{n}\right)_{n}$ of finite words which converges to $v$ such that $c\left(w_{n}\right)=c(v)$ for all $n$. Hence it suffices to establish that $\mathrm{R} \models u v=u$ in case $v$ is a finite word. This in turn follows directly from the definition of cumulative content by induction on the length of the word. 
Conversely, suppose that $\mathrm{R} \models u v=u$. If $c(v) \nsubseteq \vec{c}(u)$ then there is some factorization of the form $v=v_{0} a v_{1}$ such that $c\left(v_{0}\right) \subseteq \vec{c}(u)$ and $a \notin \vec{c}(u)$. By the already proved half of the lemma, we have $\mathrm{R} \models u v_{0}=u$. Hence $\mathrm{R} \models u a v_{1}=u$. Hence, in finite $\mathcal{R}$-trivial semigroups, $u a$ and $u$ are $\mathcal{R}$ equivalent, and therefore they are equal. Hence, $\mathrm{R} \models u a=u$, which yields $a \in \vec{c}(u)$, in contradiction with the choice of $a$. Hence $c(v) \subseteq \vec{c}(u)$.

The last sentence in the statement of the lemma is now immediate taking into account that we always have $\vec{c}(u) \subseteq c(u)$.

We will also need the following well-known property of R (cf. 2] Section 9.2]).

Lemma 4.4 Let $w$ be a pseudoword and let a be a letter such that, for every positive integer $n$, there exists a factorization $w=w_{1} \cdots w_{n}$ with $a \in$ $c\left(w_{n}\right) \subseteq \cdots \subseteq c\left(w_{1}\right)$. Then $a \in \vec{c}(w)$.

We will say that a pseudoword is end-marked if it is of the form $u a$ where $a$ is a letter and $u$ is a pseudoword such that $a \notin \vec{c}(u)$. The following result states that the $\mathcal{R}$-equivalence is trivial on end-marked pseudowords.

Lemma 4.5 If ua and $v b$ are end-marked pseudowords such that ua $\mathcal{R} v b$, then $a=b$ and $u=v$.

Proof. By hypothesis there are possibly empty pseudowords $x$ and $y$ such that $v b x=u a$ and $u a y=v b$. If $y=1$ then the result follows from Corollary 4.2](2), Otherwise, also by Corollary 4.2](2), from $u a y=v b$ we deduce that there is a factorization of the form $y=y^{\prime} b$ and $u a y^{\prime}=v$. Similarly, we may assume that there is a factorization $x=x^{\prime} a$ such that $v b x^{\prime}=u$. Hence $u=u \cdot a y^{\prime} b x^{\prime}=u\left(a y^{\prime} b x^{\prime}\right)^{n}$ for all $n \geq 1$ which implies that $u=u\left(a y^{\prime} b x^{\prime}\right)^{\omega}$. Since $\mathrm{R} \models(a z)^{\omega} a=(a z)^{\omega}$, we deduce that $\mathrm{R} \models u a=u$. This contradicts the hypothesis that $a \notin \vec{c}(u)$ and therefore we must have $y=1$, which establishes the lemma.

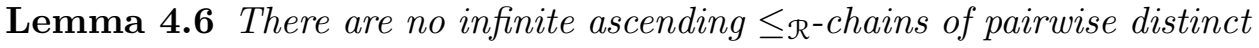
end-marked pseudowords over a finite alphabet.

Proof. Suppose that $u_{1} a_{1} \leq_{\mathcal{R}} u_{2} a_{2} \leq_{\mathcal{R}} \cdots$ is an ascending chain of endmarked pseudowords. By Lemma 4.5, $\mathcal{R}$-equivalent terms in the chain must be equal. Hence we may assume that the sequence is strictly increasing. Arguing by contradiction, we assume also that the chain is infinite.

Then, for each $n \geq 2$, there is some nonempty pseudoword $x_{n}$ such that $u_{n} a_{n} x_{n}=u_{n-1} a_{n-1}$. Cancelling the last letter, we obtain a factorization of the form $u_{n-1}=u_{n} a_{n} y_{n}$. By finiteness of the alphabet and by compactness of $\bar{\Omega}_{A} \mathrm{~S}$, we may replace the chain by an infinite subchain such that each of the sequences $\left(a_{n}\right)_{n},\left(\vec{c}\left(u_{n}\right)\right)_{n}$, and $\left(c\left(a_{n} y_{n}\right)\right)_{n}$ is constant, and the sequences $\left(u_{n}\right)_{n}$ and $\left(a_{n} y_{n} \cdots a_{2} y_{2}\right)_{n}$ converge, say respectively to the pseudowords $u$ 
and $v$. Let $a$ be the letter in the sequence $\left(a_{n}\right)_{n}$. Since $v$ is a product of arbitrarily many factors with the same content, Lemma 4.4 implies that $\mathrm{R} \models v a=v$. Hence $a \in \vec{c}(v)$. Since $u_{1}=u_{n} a_{n} y_{n} \cdots a_{2} y_{2}$ for all $n$, by continuity of multiplication we obtain the equality $u_{1}=u v$. Hence $a_{1}=$ $a \in \vec{c}(v) \subseteq \vec{c}\left(u_{1}\right)$ which is a contradiction since $u_{1} a_{1}$ is assumed to be an end-marked pseudoword.

We next recall from [31, Chapter XII] some important properties of right Rhodes expansions. Given a semigroup $S$, its Rhodes expansion is given by an onto homomorphism $\eta_{S}: \hat{S}^{\mathcal{R}} \rightarrow S$. If $S$ is $A$-generated, in the sense that a mapping $\iota: A \rightarrow S$ is given (the choice of generators) whose image generates $S$, then one may consider the subsemigroup of $\hat{S}^{\mathcal{R}}$ which is $A$ generated via the mapping $\rho_{S}: A \rightarrow \hat{S}^{\mathcal{R}}$ such that $\eta_{S} \circ \rho_{S}=\iota$ [26]. This gives a version of the right Rhodes expansion which is said to be cutdown to generators. It is denoted $\hat{S}_{A}^{\mathcal{R}}$, and it has, among others, the following property [26, Fact 2.8]:

$$
\begin{aligned}
& \text { If } r, s, t \in \hat{S}_{A}^{\mathcal{R}} \text { are such that } r \geq_{\mathcal{R}} t \text { and } s \geq_{\mathcal{R}} t \text {, then } r \geq_{\mathcal{R}} s \\
& \text { or } s \geq_{\mathcal{R}} r \text {. }
\end{aligned}
$$

Let LZ be the pseudovariety of finite left-zero semigroups, which is defined by the identity $x y=x$. Then, using the same sort of argument as in the proof of [17. Proposition 3.3], it is easy to show that every pseudovariety of the form LZ $m \mathrm{~V}$ is closed under taking right Rhodes expansions. On the other hand, it is a routine calculation to check that $L Z m R=R$. It follows that $\mathrm{R}$ is closed under right Rhodes expansions, a conclusion which could also be drawn for example by invoking [31, Corollary XII.12.2].

Lemma 4.7 Let $\mathrm{V}$ be a pseudovariety of semigroups which is closed under right Rhodes expansions. Suppose that $u$ and $v$ are two prefixes of the same element of $\bar{\Omega}_{A} \mathrm{~V}$. Then one of $u$ and $v$ is a prefix of the other.

Proof. Let $\left(S_{n}\right)_{n}$ be a sequence of $A$-generated semigroups from $\mathrm{V}$ such that every other $A$-generated semigroup from $\mathrm{V}$ is a homomorphic image of $S_{n}$, by a homomorphism which respects the choice of generators, for all sufficiently large $n$ (cf. [18]). Then the sequence $\left({\widehat{S_{n}}}_{A}^{\mathcal{R}}\right)_{n}$ possesses the same property. Moreover, note that every infinite subsequence retains yet the same property. Let $\psi_{n}: \bar{\Omega}_{A} \mathrm{~V} \rightarrow{\widehat{S_{n}}}_{A}^{\mathcal{R}}$ be the unique continuous homomorphism such that $\psi_{n} \circ \iota_{\mathrm{V}}=\rho_{S_{n}}$ where $\iota_{\mathrm{V}}: A \rightarrow \bar{\Omega}_{A} \mathrm{~V}$ is the natural mapping.

From the hypothesis that $u$ and $v$ are prefixes of the same element of $\bar{\Omega}_{A} \mathrm{~V}$ it follows that $\psi_{n}(u)$ and $\psi_{n}(v)$ are both $\mathcal{R}$-above the same element of $\widehat{S_{n A}}$. By (4.1), we deduce that $\psi_{n}(u)$ and $\psi_{n}(v)$ are comparable under $\leq \mathcal{R}$. By taking a subsequence, we may assume that $\psi_{n}(u) \leq_{\mathcal{R}} \psi_{n}(v)$ for every $n$. By a standard compactness argument, we conclude that we may assume that $u \leq_{\mathcal{R}} v$ (cf. [2, Section 5.6] and [6, Section 8]). 
We say that a partially ordered set $(Y, \leq)$ is a forest if the set of elements above any given element is a chain, and we say it is well-founded if it has no infinite ascending chains.

The following result is now immediate in view of Lemmas 4.5, 4.6, and 4.7 since the pseudovariety $\mathrm{S}$ is obviously closed under right Rhodes expansions.

Proposition 4.8 The set of all end-marked pseudowords over a finite alphabet constitutes a well-founded forest under the partial order $\leq_{\mathcal{R}}$.

The following is another useful property involving the cumulative content. Indirect proofs can be derived from known structure theorems for $\bar{\Omega}_{A} \mathrm{R}$. The direct proof which we provide is inspired by the proof of [2, Lemma 9.2.10]. For a positive integer $m$ we denote by $E_{m}$ the semigroup of all expansive transformations (acting on the right) of the set $\{1, \ldots, m\}$ into itself, that is such transformations $f$ which satisfy if $\geq i$ for all $i$. It is well known that a finite semigroup is $\mathcal{R}$-trivial if and only if it may be embedded in some $E_{m}$. See [2, Proposition 9.2.5] for a direct proof, or [3. Chaper 4, Theorem 3.3] for a proof based on Eilenberg's characterization of the variety of languages corresponding to R [31, Chapter X, Corollary 3.3].

Lemma 4.9 Let $u=u_{0} a u_{1}$ and $v=v_{0} b v_{1}$ be factorizations in $\bar{\Omega}_{A} \mathrm{~S}$ such that $a \in A \backslash \vec{c}\left(u_{0}\right), b \in A \backslash \vec{c}\left(v_{0}\right)$, and $\mathrm{R}$ satisfies the pseudoidentities $u_{0}=v_{0}$ and $u=v$. Then $a=b$.

Proof. Denote by $p_{\mathrm{R}}$ the unique continuous homomorphism $\bar{\Omega}_{A} \mathrm{~S} \rightarrow \bar{\Omega}_{A} \mathrm{R}$ which respects the choice of generators. By Lemma 4.7 since $p_{\mathrm{R}}\left(u_{0} a\right)$ and $p_{\mathrm{R}}\left(u_{0} b\right)=p_{\mathrm{R}}\left(v_{0} b\right)$ are both prefixes of $p_{\mathrm{R}}(u)=p_{\mathrm{R}}(v)$, we may assume that $p_{\mathrm{R}}\left(u_{0} a\right) \leq_{\mathcal{R}} p_{\mathrm{R}}\left(u_{0} b\right)$. Since R satisfies $u_{0}=v_{0}$, we have $\vec{c}\left(u_{0}\right)=\vec{c}\left(v_{0}\right)$. Since $b \notin \vec{c}\left(v_{0}\right)=\vec{c}\left(u_{0}\right)$, there exists $m$ such that $E_{m}$ fails the pseudoidentity $u_{0} b=u_{0}$. Let $\theta: \bar{\Omega}_{A} \mathrm{~S} \rightarrow E_{m}$ be a continuous homomorphism such that $\theta\left(u_{0} b\right) \neq \theta\left(u_{0}\right)$. Then $\theta\left(u_{0} a\right) \leq_{\mathcal{R}} \theta\left(u_{0} b\right)<_{\mathcal{R}} \theta\left(u_{0}\right)$. Let $i \in\{1, \ldots, m\}$ be such that, for $j=i \theta\left(u_{0}\right)$ and $k=j \theta(b)$, we have $j \neq k$. Since $E_{m}$ consists of expansive transformations, it follows that $j<k$ and $k \leq j \theta(a)$.

Suppose that $a \neq b$. We define a new continuous homomorphism $\theta^{\prime}$ : $\bar{\Omega}_{A} \mathrm{~S} \rightarrow E_{m}$ by its restriction to $A$ as follows. On $A \backslash\{a\}, \theta^{\prime}$ coincides with $\theta$. To obtain the new transformation $\theta^{\prime}(a)$ from $\theta(a)$, we only modify its value on $j$ by letting $j \theta^{\prime}(a)=j$. Since $i \theta\left(u_{0}\right)=j$ and $j<j \theta(a)$, there can be no prefix of $u_{0}$ of the form $w a$ such that $i \theta(w)=j$. Hence $i \theta^{\prime}\left(u_{0}\right)=i \theta\left(u_{0}\right)$ and, therefore, we have $i \theta^{\prime}\left(u_{0} a\right)<i \theta^{\prime}\left(u_{0} b\right)$, which contradicts the relation $\theta^{\prime}\left(u_{0} a\right) \leq_{\mathcal{R}} \theta^{\prime}\left(u_{0} b\right)$ since transformations in $E_{m}$ are expansive.

Several representations have been proposed for the elements of $\bar{\Omega}_{A} \mathrm{R}$ [19, 21. For our present purposes, the most convenient one is by reduced labeled ordinals [19. We consider labelings $\lambda: \alpha \rightarrow A$ of ordinals $\alpha$ by the alphabet 
$A$ such that the following property holds:

if $\beta$ is a limit ordinal such that $\beta<\alpha$ and there is a cofinal set $C$ of ordinals $\gamma<\beta$ such that $\lambda(\gamma)=a$ for a fixed letter $a$, then $\lambda(\beta) \neq a$.

This property implies an upper bound on the ordinal $\alpha$, namely $\alpha<\omega^{|A|}$. We denote by $\mathcal{O}_{A}$ the set of all $A$-labeled ordinals and we define a mapping $p: \bar{\Omega}_{A} \mathrm{~S} \rightarrow \mathcal{O}_{A}$ as follows.

For each pseudoword $u \in \bar{\Omega}_{A} \mathrm{~S}$, we consider the set of all end-marked pseudowords $v a$ which are prefixes of $u$. By Proposition 4.8, this set is well ordered under the relation $\geq_{\mathcal{R}}$ and, therefore, there is a unique isomorphism $\theta$ from an ordinal $\alpha_{u}$ onto it. We define the labeling $\lambda_{u}: \alpha_{u} \rightarrow A$ by letting $\lambda_{u}(\beta)$ be the last letter of $\theta(\beta)$. Observe that, by definition of $\theta, \lambda_{u}$ satisfies (4.2). The mapping $p: \bar{\Omega}_{A} \mathrm{~S} \rightarrow \mathcal{O}_{A}$ sends $u$ to the ordinal $\alpha_{u}$ labeled by $\lambda_{u}$.

The following result is a reformulation of [19, Theorem 3.3.5]. We present a direct proof to justify the above description of the labeled ordinal associated with a pseudoword.

Theorem 4.10 The mapping $p: \bar{\Omega}_{A} \mathrm{~S} \rightarrow \mathcal{O}_{A}$ is such that, for all $u, v \in \bar{\Omega}_{A} \mathrm{~S}$, $p(u)=p(v)$ if and only if $\mathrm{R} \models u=v$.

Proof. Suppose first that $p(u)$ and $p(v)$ are given by the same ordinal $\alpha$ and $\lambda_{u}=\lambda_{v}$. Proceeding by transfinite induction on $\alpha$, to show that $\mathrm{R} \models u=v$, we may assume that the direct implication of the statement of the theorem holds whenever the ordinal involved is smaller than $\alpha$. Note that $u$ is an end-marked pseudoword if and only if $\alpha$ is a successor ordinal. Hence, if $\alpha=\beta+1$ is a successor ordinal, $u=u^{\prime} a$ and $v=v^{\prime} b$ are both end-marked pseudowords. Since $\lambda_{u}=\lambda_{v}$, it follows that $a=b$. Moreover, since $u^{\prime} a$ and $v^{\prime} a$ are end-marked, we obtain $\alpha_{u^{\prime}}=\beta=\alpha_{v^{\prime}}$. Finally, since $\lambda_{u}=\lambda_{v}$, we have $\lambda_{u^{\prime}}=\left.\lambda_{u}\right|_{\beta}=\left.\lambda_{v}\right|_{\beta}=\lambda_{v^{\prime}}$. Applying the induction hypothesis, we deduce that $\mathrm{R} \models u^{\prime}=v^{\prime}$ and so also $\mathrm{R} \models u=v$.

Consider next the case where $\alpha$ is a limit ordinal. Since $\alpha$ is countable, there is an increasing sequence of ordinals $\left(\beta_{n}\right)_{n}$ whose union is $\alpha$. We may assume that the sequence of the $\lambda_{u}\left(\beta_{n}\right)=\lambda_{v}\left(\beta_{n}\right)$ is constant, say with value $a$. Let $\psi$ and $\theta$ be the isomorphisms of $\alpha$ with the sets of end-marked prefixes of $u$ and $v$, respectively, and let $\psi\left(\beta_{n}\right)=u_{n} a$ and $\theta\left(\beta_{n}\right)=v_{n} a$. Then we may also assume that the sequences $\left(u_{n}\right)_{n}$ and $\left(v_{n}\right)_{n}$ converge, say to the respective limits $u^{\prime}$ and $v^{\prime}$ where there are factorizations $u=u^{\prime} u^{\prime \prime}$ and $v=v^{\prime} v^{\prime \prime}$. From the induction hypothesis, we have $\mathrm{R} \models u_{n}=v_{n}$ for every $n$. Hence $\mathrm{R} \models u^{\prime}=v^{\prime}$.

To conclude the induction step, in view of Lemma 4.3 it suffices to show that $c\left(u^{\prime \prime}\right) \subseteq \vec{c}\left(u^{\prime}\right)$. If this were not the case, then there would be a letter $b \in c\left(u^{\prime \prime}\right) \backslash \vec{c}\left(u^{\prime}\right)$ and a factorization $u^{\prime \prime}=x b y$ with $c(x) \subseteq \vec{c}\left(u^{\prime}\right)$. Then $u^{\prime} x b$ is an end-marked prefix of $u$ since, by Lemma 4.3. $\vec{c}\left(u^{\prime} x\right)=\vec{c}\left(u^{\prime}\right)$. Since $\alpha$ 
is the union of the $\beta_{n}$, there must exist an end-marked prefix $w d$ of some $u_{n} a$ such that $w d=u^{\prime} x b$, which, by Corollary 4.2](2) implies that $d=b$ and $w=u^{\prime} x$. But $u_{n} a$ is a prefix of $u^{\prime}$ and therefore so is $w b$, say $u^{\prime}=w b z$. Hence $w=u^{\prime} x=w b z x$ which implies that $b \in \vec{c}(w)$. This contradicts the choice of $b$ since $\vec{c}(w)=\vec{c}\left(u^{\prime} x\right)=\vec{c}\left(u^{\prime}\right)$.

For the converse, assume that $\mathrm{R} \models u=v$. Without loss of generality, we may also assume that $\alpha_{u} \leq \alpha_{v}$. We first prove, by transfinite induction on $\beta<\alpha_{u}$, that $\lambda_{u}(\beta)=\lambda_{v}(\beta)$. Assume then that the equality $\lambda_{u}(\gamma)=\lambda_{v}(\gamma)$ holds for every $\gamma<\beta$. Let $u=u_{0} a u_{1}$ and $v=v_{0} b v_{1}$ be factorizations such that $u_{0} a$ and $v_{0} b$ are the end-marked prefixes corresponding to $\beta$ in the unique isomorphisms between the $\geq_{\mathcal{R}}$-ordered sets of such prefixes of $u$ and $v$ and the labeled ordinals $p(u)$ and $p(v)$, respectively. Then the ordinal associated with each of $u_{0}$ and $v_{0}$ is $\beta$ and, by the induction hypothesis, for both it is labeled by the same function, namely $\left.\lambda_{u}\right|_{\beta}=\left.\lambda_{v}\right|_{\beta}$. By the direct part of the statement of the theorem, it follows that $\mathrm{R} \models u_{0}=v_{0}$. By Lemma 4.9. we deduce that $a=b$, that is that $\lambda_{u}(\beta)=\lambda_{v}(\beta)$. This completes the induction step. Hence $\lambda_{u}(\beta)=\lambda_{v}(\beta)$ for all $\beta<\alpha_{u}$ and therefore there is nothing else to prove in case $\alpha_{u}=\alpha_{v}$.

Suppose next that $\alpha_{u}<\alpha_{v}$. Let now $v=v_{0} a v_{1}$ be a factorization such that $v_{0} a$ is the end-marked prefix of $v$ corresponding to $\alpha_{u}$ in the unique isomorphism between $\alpha_{v}$ and the $\geq_{\mathcal{R}^{\text {-ordered }}}$ set of end-marked prefixes of $v$. Since $\lambda_{u}=\left.\lambda_{v}\right|_{\alpha_{u}}$ by the above, it follows that $p(u)=p\left(v_{0}\right)$ and so again $\mathrm{R} \models u=v_{0}$ by the already-proved direct part of the statement of the theorem. Hence $\mathrm{R} \models v_{0}=u=v=v_{0} a v_{1}$ which, by Lemma 4.3 , yields $a \in \vec{c}\left(v_{0}\right)$. This is in contradiction with the assumption that $v_{0} a$ is an end-marked pseudoword and, therefore, we conclude that $\alpha_{u}=\alpha_{v}$ and so $p(u)=p(v)$.

For ordinals $\beta_{1}$ and $\beta_{2}$ such that $\beta_{1}<\beta_{2}$, we denote by $\beta_{2}-\beta_{1}$ the unique ordinal $\gamma$ such that $\beta_{1}+\gamma=\beta_{2}$.

Lemma 4.11 Let $\beta_{1}, \gamma_{1}, \beta_{2}, \gamma_{2}$ be ordinals such that $0 \leq \beta_{1}<\gamma_{1}$ and $0 \leq$ $\beta_{2}<\gamma_{2}$. If $\gamma_{1}<\gamma_{2}$ and $\gamma_{1}-\beta_{1}=\gamma_{2}-\beta_{2}$, then $\beta_{1}<\beta_{2}$.

Proof. By elementary results on ordinal arithmetic, if $\beta_{1} \geq \beta_{2}$, then we obtain $\gamma_{1}=\beta_{1}+\left(\gamma_{1}-\beta_{1}\right) \geq \beta_{2}+\left(\gamma_{2}-\beta_{2}\right)=\gamma_{2}$, a contradiction.

Given two labeled ordinals $\lambda: \alpha \rightarrow A$ and $\mu: \beta \rightarrow A$, where $\alpha$ is not a limit, define their concatenation $\lambda \cdot \mu$ to be the ordinal sum $\alpha+\beta$ labeled by

$$
\gamma \mapsto \begin{cases}\lambda(\gamma) & \text { if } \gamma<\alpha \\ \mu(\gamma-\alpha) & \text { if } \gamma \geq \alpha .\end{cases}
$$

Then we have the following result.

Lemma 4.12 If $w_{0}$ a is an end-marked pseudoword then we have the equality $p\left(w_{0} a w_{1}\right)=p\left(w_{0} a\right) \cdot p\left(w_{1}\right)$. 
Proof. It suffices to observe that, in view of Proposition 4.1, the end-marked prefixes of $w_{0} a w_{1}$ of which $w_{0} a$ is a prefix are of the form $w_{0} a w_{2} b$ where $w_{2} b$ is an end-marked prefix of $w_{1}$.

As an application of Theorem 4.10, we can now add a refined conclusion to Lemma 4.9

Corollary 4.13 Let $u=u_{0} a u_{1}$ and $v=v_{0} b v_{1}$ be factorizations in $\bar{\Omega}_{A} \mathrm{~S}$ such that $a \in A \backslash \vec{c}\left(u_{0}\right), b \in A \backslash \vec{c}\left(v_{0}\right)$, $\mathrm{R}$ satisfies the pseudoidentity $u=v$, and $\alpha_{u_{0}}=\alpha_{v_{0}}$. Then $a=b$ and $\mathrm{R}$ satisfies the pseudoidentities $u_{0}=v_{0}$ and $u_{1}=v_{1}$.

Proof. By Lemma 4.12 $p(u)=p\left(u_{0} a\right) \cdot p\left(u_{1}\right)$ and $p(v)=p\left(v_{0} b\right) \cdot p\left(v_{1}\right)$. From the hypothesis that $\mathrm{R} \models u=v$, by Theorem 4.10 we deduce that $p(u)=p(v)$. Taking into account the definition of concatenation of labeled ordinals, it follows that $p\left(u_{0}\right)=p\left(v_{0}\right), a=b$, and $p\left(u_{1}\right)=p\left(v_{1}\right)$. Invoking again Theorem 4.10, we obtain the corollary.

Yet another improvement of Lemma 4.9 is given by the following result.

Corollary 4.14 Let $x, y, z, t$ be pseudowords over a finite alphabet $A$ and $a, b \in A$ be letters such that $x a y=z b t, x a$ and $z b$ are end-marked, and $\mathrm{R} \models x=z$. Then $x=z, a=b$, and $y=t$.

Proof. Both $x a$ and $z b$ are prefixes of $x a y=z b t$. By Lemma 4.7 applied to $\mathrm{S}$ (which is clearly closed under right Rhodes expansions), $x a$ and $z b$ are $\leq_{\mathcal{R}^{-}}$ comparable. Assume that $x a \neq z b$. By symmetry, one can assume without loss of generality that $x a$ is a proper prefix of $z b$, so that is $z=x a x^{\prime}$ for some $x^{\prime} \in\left(\bar{\Omega}_{A} \mathrm{~S}\right)^{1}$. Then we have $\alpha_{z} \geq \alpha_{x a}=\alpha_{x}+1$ since $x a$ is end-marked. In particular, $p(z) \neq p(x)$, so that by Theorem 4.10, we deduce $\mathrm{R} \not \models x=z$, in contradiction with the hypothesis of the statement. Hence $x a=z b$. By the dual of Proposition 4.1. we conclude that $x=z$ and $a=b$. It remains to show that $y=t$.

By hypothesis, $\mathrm{R}$ fails the pseudoidentity $x a=x$. Hence there exists a positive integer $m$, a continuous homomorphism $\psi: \bar{\Omega}_{A} S \rightarrow E_{m}$, and $i \in Y$, where $Y=\{1, \ldots, m\}$, such that $i \psi(x a) \neq i \psi(x)$. Since $E_{m}$ consists of expansive transformations, we must have $j<j \psi(a)$ for $j=i \psi(x)$. Suppose that $y \neq t$. Then there exists a finite set $Z$ disjoint from $Y$, a continuous homomorphism $\theta: \bar{\Omega}_{A} S \rightarrow T_{Z}$ into the semigroup $T_{Z}$ of all transformations of the set $Z$, and a point $q \in Z$ such that $q \theta(y) \neq q \theta(t)$. We define a continuous homomorphism $\tau: \bar{\Omega}_{A} \mathrm{~S} \rightarrow T_{Y \cup Z}$ by letting, for each letter $d \in A \backslash\{a\}$ and each $r \in Y \cup Z, r \tau(d)$ be $r \psi(d)$ or $r \theta(d)$ according to whether $r \in Y$ or $r \in Z$; in case $d=a$, we also use the same rule unless $r=j$, for we let $j \tau(a)=q$. Then we still have $i \tau(x)=j$ and $q \tau(y) \neq q \tau(t)$ so that

$$
i \tau(x a y)=j \tau(a y)=q \tau(y) \neq q \tau(t)=j \tau(a t)=i \tau(x a t)
$$


which shows that $\tau(x a y) \neq \tau(x a t)$ and, therefore, that $x a y \neq x a t$, which is in contradiction with the assumption. This shows that $y=t$.

Corollary 4.14 justifies the following notation which will be very useful in the sequel. Let $w$ be a pseudoword and let $\beta$ and $\gamma$ be ordinals such that $\beta \leq \gamma<\alpha_{w}$. Let $x a$ and $y b$ be end-marked prefixes of $w$ corresponding to $\beta$ and $\gamma$, respectively. If $\beta<\gamma$ then $x a$ is a prefix of $y$ and, by Corollary 4.14, there is a unique pseudoword $z$ such that $y=x a z$. We denote $z, a z, z b$, and $a z b$ respectively by $w] \beta, \gamma\left[, w[\beta, \gamma[, w] \beta, \gamma]\right.$, and $w[\beta, \gamma]$. In case $\beta<\gamma=\alpha_{w}$, we let $w[\beta, \gamma[=a z$ and $w] \beta, \gamma[=z$, where $w=x a z$ and $x a$ is the end-marked prefix corresponding to $\beta$. For the case $\gamma=\beta$, we also set $w] \beta, \beta[, w[\beta, \beta[$, and $w] \beta, \beta]$ to be the empty word, and $w[\beta, \beta]=\lambda_{w}(\beta)$. Note that

$$
\beta_{1} \leq \beta_{2} \leq \beta_{3} \leq \alpha_{w} \Longrightarrow w\left[\beta_{1}, \beta_{3}\left[=w\left[\beta_{1}, \beta_{2}\left[w \left[\beta_{2}, \beta_{3}[.\right.\right.\right.\right.\right.
$$

Example 4.15 Let $w=(a b)^{\omega} a c a^{\omega}$. Then $\alpha_{w}=\omega 2$ and the end-marked prefixes of $w$ corresponding to the ordinals $\omega$ and $\omega+3$ are respectively $(a b)^{\omega} a c$ and $(a b)^{\omega} a c a^{3}$. Hence

$$
w\left[0, \omega\left[=(a b)^{\omega} a ; \quad w\left[\omega, \omega+3\left[=c a^{2} ; \quad w\left[\omega+3, \omega 2\left[=a^{\omega-2} .\right.\right.\right.\right.\right.\right.
$$

Recall also that, by definition of $\alpha_{w}$, given ordinals $\beta, \gamma<\alpha_{w}$,

$$
\beta<\gamma \Longleftrightarrow w[0, \beta]>_{\mathcal{R}} w[0, \gamma] .
$$

In view of the equivalence (4.3), Corollary 4.14 may be viewed as a can-

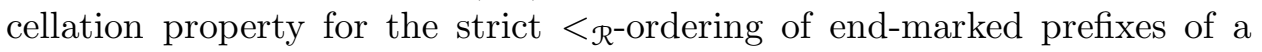
pseudoword.

\section{$5 \quad$ Multiple periods over $\mathrm{R}$}

The main result of this section, Proposition 5.5. shows a necessary and sufficient condition for idempotent pseudowords to be equal over R. It will be used in Section 9, but depends on proof techniques similar to those of Section 4 .

Lemma 5.1 Let $u$ and $v$ be pseudowords such that $\mathrm{R}$ satisfies $v u^{\omega}=u^{\omega}$ and $c(v) \varsubsetneqq c(u)$. Then $\mathrm{R}$ satisfies $v u=u$.

Proof. Let $u=u_{0} u_{1}$ with $c\left(u_{0}\right) \subseteq c(v)$, and the first letter of $u_{1}$ is not in $c(v)$. Note that since $c(v) \varsubsetneqq c(u)$, the existence and uniqueness of such a factorization of $u$ follows from Corollary [4.2[1). Since, by hypothesis, the pseudovariety $\mathrm{R}$ satisfies $v u^{\omega}=u^{\omega}$, it also satisfies $v u_{0} u_{1} u^{\omega}=u_{0} u_{1} u^{\omega}$. Hence by Theorem 4.10. $\mathrm{R}$ satisfies $v u_{0}=u_{0}$. Therefore, $v u=u$ holds in $\mathrm{R}$. 
Lemma 5.2 If $u, v \in \bar{\Omega}_{A} \mathrm{~S}$ are such that $\mathrm{R}$ satisfies the pseudoidentity $v u^{2}=$ $u^{2}$, then it also satisfies $v u=u$.

Proof. Since R satisfies the pseudoidentity $v u^{2}=u^{2}$, it also satisfies $v u^{\omega}=$ $u^{\omega}$. The case where $c(v) \varsubsetneqq c(u)$ follows from Lemma 5.1 and so we assume that $c(v)=c(u)$.

Using the pseudoidentity $u^{2}=v u^{2}$ iteratively, which is satisfied by $\mathrm{R}$, we deduce the pseudoidentity $u^{2}=v^{n} u^{2}$ for all $n$ which yields $\mathrm{R} \models u^{2}=$ $v^{\omega} u^{2}=v^{\omega}$, where we also use Lemma 4.3, Therefore, again by Lemma 4.3. $c\left(u^{2}\right)=\vec{c}\left(u^{2}\right)$. Hence $c(u)=c\left(u^{2}\right)=\vec{c}\left(u^{2}\right)=\vec{c}(u)$ and so $\mathrm{R} \models u^{2}=u$, once again by Lemma 4.3, from which the conclusion of the lemma follows.

A product of possibly empty pseudowords $w_{1} \cdots w_{n}$ is said to be reduced if, for $i=1, \ldots, n-1$, either $w_{i+1}=1$ or the first letter of $w_{i+1}$ does not belong to $\vec{c}\left(w_{i}\right)$. We will use the following remark, which follows directly from Theorem 4.10

Fact 5.3 If $u, v, w \in \bar{\Omega}_{A} \mathrm{~S}$ are pseudowords such that $\mathrm{R}$ satisfies $w=u v$ and the product $u v$ is reduced, then $w$ admits a factorization $u^{\prime} v^{\prime}$ such that $\mathrm{R}$ satisfies both $u=u^{\prime}$ and $v=v^{\prime}$.

Lemma 5.4 Let $x, y$ be pseudowords over A such that $\mathrm{R}$ satisfies $x^{\omega}=y^{\omega}$. Assume that the products $x x$ and $y y$ are reduced. Then, there exist $w \in$ $\bar{\Omega}_{A} \mathrm{~S}, r, s \in\left(\bar{\Omega}_{A} \mathrm{~S}\right)^{1}$, and integers $k, \ell>0$ such that $\mathrm{R}$ satisfies the following pseudoidentities:

$$
\left\{\begin{array}{l}
x=w^{k} r \\
y=w^{\ell} s \\
w=r w=s w
\end{array}\right.
$$

where all the indicated products as well as ww are reduced.

Proof. If R satisfies $x=y$, then we can choose $w=x, k=\ell=1$, and $r=s=1$. From now on, we assume that $\mathrm{R}$ does not satisfy $x=y$.

We argue by transfinite induction on $\max \left\{\alpha_{x}, \alpha_{y}\right\}$. Assume that the result holds when $\max \left\{\alpha_{x}, \alpha_{y}\right\}<\alpha$ and let $\max \left\{\alpha_{x}, \alpha_{y}\right\}=\alpha$. Assume next that $\alpha_{x}=\alpha_{y}$. Since R satisfies $x x^{\omega}=y y^{\omega}$, we would then have $p(x)=p(y)$, so that $\mathrm{R}$ would satisfy $x=y$, a case that we have already excluded. Hence $\alpha_{x} \neq \alpha_{y}$ and, by symmetry, we can assume without loss of generality that $\alpha_{x}<\alpha_{y}=\alpha$.

Since $x^{\omega}=\mathrm{R} y^{\omega}$ by hypothesis, we deduce that $\mathrm{R}$ satisfies $x^{\omega}=y x^{\omega}$. Hence, $x^{n} x^{\omega}=\mathrm{R}_{\mathrm{R}} y x^{\omega}$ for every positive integer $n$. Since $\alpha_{x}<\alpha_{y}$, there is, by Fact [5.3. some reduced factorization $y=y_{1} y_{1}^{\prime}$ where $y_{1}=_{\mathrm{R}} x$, and therefore the set

$$
J=\left\{n \geq 1:\left(\exists y_{1}, \ldots, y_{n}\right) y \leq_{\mathcal{R}} y_{1} \cdots y_{n} \text { and }(\forall i, 1 \leq i \leq n) y_{i}={ }_{\mathrm{R}} x\right\}
$$


is nonempty. If it were unbounded, then by Lemma 4.4. any letter of $c(x)=$ $c\left(y_{i}\right)(i \geq 1)$ would be in the cumulative content of $y$, so that we would have $\vec{c}(y)=c(x)=c(y)$, a contradiction with the hypothesis that the first letter of $y$ is not in $\vec{c}(y)$. Let $m$ be the maximum of the set $J$. Then we can write $y$ as a reduced product

$$
y=y_{1} \cdots y_{m} y^{\prime} \text { with } y_{i}={ }_{\mathrm{R}} x \text { for } i \geq 1 .
$$

From $x^{\omega}={ }_{\mathrm{R}} y x^{\omega}$ and (5.1), using Corollary 4.13, we deduce also that

$$
\mathrm{R} \models x^{\omega}=y^{\prime} x^{\omega} .
$$

We now distinguish two cases.

- If $c\left(y^{\prime}\right) \varsubsetneqq c(x)$, then by (5.2) and Lemma [5.1. R satisfies $x=y^{\prime} x$, so that one can choose $w=x, k=1, \ell=m, r=1, s=y^{\prime}$.

- If $c\left(y^{\prime}\right)=c(x)$, then (5.2) shows that $\mathrm{R}$ satisfies $x^{\omega}=y^{\prime \omega}$. Further, $\vec{c}\left(y^{\prime}\right)=\vec{c}(y)$ and the first letter of $y^{\prime}$ is the first letter of $x$, that is, the first letter of $y$. Since by hypothesis the first letter of $y$ is not in $\vec{c}(y)$, we deduce that the first letter of $y^{\prime}$ is not in $\vec{c}\left(y^{\prime}\right)$. Therefore the pair $\left(y^{\prime}, x\right)$ satisfies the hypotheses of the lemma. By maximality of $m$, we obtain $\alpha_{y^{\prime}} \leq \alpha_{x}<\alpha_{y}=\alpha$, so we can apply the induction hypothesis to deduce that there exist $w \in \bar{\Omega}_{A} \mathrm{~S}, k, \ell>0$ and $r, s \in\left(\bar{\Omega}_{A} \mathrm{~S}\right)^{1}$ such that R satisfies the following pseudoidentities

$$
\left\{\begin{array}{l}
x=w^{k} r \\
y^{\prime}=w^{\ell} s \\
w=r w=s w
\end{array}\right.
$$

where all the products and $w w$ are reduced. Therefore, $\mathrm{R}$ satisfies $y=$ $x^{m} y^{\prime}=w^{k m+\ell} s$.

This completes the proof of the lemma.

More generally, we have the following result which will be crucial in the proof of the main theorem of this paper.

Proposition 5.5 Let $x_{0}, \ldots, x_{n}$ be pseudowords over $A$ such that $\mathrm{R}$ satisfies $x_{0}^{\omega}=\cdots=x_{n}^{\omega}$. Assume that, for all $i$, the product $x_{i} x_{i}$ is reduced. Then, there exist $u \in \bar{\Omega}_{A} S, v_{i} \in\left(\bar{\Omega}_{A} S\right)^{1}$, and integers $m_{i}>0$ such that $\mathrm{R}$ satisfies the following pseudoidentities, for all $i=0, \ldots, n$,

$$
\left\{\begin{array}{l}
x_{i}=u^{m_{i}} v_{i} \\
u=v_{i} u
\end{array}\right.
$$

where all the products and uu are reduced. 
Proof. We prove the result by induction on $n$. The case $n=1$ has already been treated in Lemma [5.4. Let $x_{0}, \ldots, x_{n}$ be pseudowords satisfying the hypotheses of the proposition. By Lemma 5.4 applied to each pair $\left(x_{0}, x_{i}\right)$, $i=1, \ldots, n$, we obtain $w_{i} \in \bar{\Omega}_{A} \mathrm{~S}, r_{i}, s_{i} \in\left(\bar{\Omega}_{A} \mathrm{~S}\right)^{1}$ and integers $k_{i}, \ell_{i}>0$ such that $\mathrm{R}$ satisfies the pseudoidentities

$$
\left\{\begin{array}{l}
x_{0}=w_{i}^{k_{i}} r_{i} \\
x_{i}=w_{i}^{\ell_{i}} s_{i} \\
w_{i}=r_{i} w_{i}=s_{i} w_{i}
\end{array}\right.
$$

where all the products, as well as $w_{i} w_{i}$, are reduced. Since $x_{0}^{\omega}=w_{1}^{\omega}=$ $\cdots=w_{n}^{\omega}$, we can apply the induction hypothesis for $w_{1}, \ldots, w_{n}$ to obtain $y \in \bar{\Omega}_{A} \mathrm{~S}, z_{i} \in\left(\bar{\Omega}_{A} \mathrm{~S}\right)^{1}$, and integers $n_{i}>0$ such that R satisfies the following pseudoidentities:

$$
\left\{\begin{array}{l}
w_{i}=y^{n_{i}} z_{i} \\
y=z_{i} y
\end{array}\right.
$$

where all the products and yy are reduced. Using (5.3), we deduce that R satisfies

$$
\begin{aligned}
x_{0} & =\left(y^{n_{i}} z_{i}\right)^{k_{i}} r_{i}=y^{n_{i} k_{i}} z_{i} r_{i}, \\
x_{i} & =\left(y^{n_{i}} z_{i}\right)^{\ell_{i}} s_{i}=y^{n_{i} \ell_{i}} z_{i} s_{i} .
\end{aligned}
$$

Setting $u=y, v_{0}=z_{1} r_{1}, v_{i}=z_{i} s_{i}$ for $i \geq 1, m_{0}=n_{1} k_{1}$ and $m_{i}=n_{i} \ell_{i}$ for $i \geq 1$, it remains to show that $\mathrm{R}$ satisfies

$$
y=z_{1} r_{1} y=z_{i} s_{i} y
$$

From (5.3), we have $\mathrm{R} \models w_{i}=r_{i} w_{i}$, that is $\mathrm{R} \models y^{n_{i}} z_{i}=r_{i} y^{n_{i}} z_{i}$. Multiplying by $y^{2^{n_{i}}-n_{i}}$ on the right and using $\mathrm{R} \models y=z_{i} y$, we obtain $\mathrm{R} \models y^{2^{n_{i}}}=r_{i} y^{2^{n_{i}}}$, which yields $\mathrm{R} \models y=r_{i} y$, by Lemma [5.2. Multiplying on the left by $z_{i}$ and using (5.4), we therefore obtain $\mathrm{R} \models y=z_{i} r_{i} y$, the first pseudoidentity of (5.5) for $i=1$. The other pseudoidentity can be obtained similarly.

\section{Further simplifications for $\mathrm{R}$}

We present some further simplifications in the setup of the $\kappa$-reducibility problem introduced at the end of Section 3 which may be applied for the pseudovariety R.

Note that $\vec{c}\left(x^{\omega}\right)=c(x)$ for every pseudoword $x$. The following simple lemma will allow us to work with word equations instead of $\kappa$-term equations.

Lemma 6.1 Let $u$ and $v$ be pseudowords. Then $\mathrm{R}$ satisfies the pseudoidentity $u^{\omega}=v$ if and only if $\mathrm{R}$ satisfies the pseudoidentity $u v=v$ and $c(u)=$ $c(v)$. 
Proof. From the assumption that $\mathrm{R} \models u^{\omega}=v$, we deduce that the semilattice $\{0,1\}$ satisfies $u=v$, which implies that $c(u)=c(v)$. Moreover, the same assumption implies that $\mathrm{R} \models u v=u u^{\omega}=u^{\omega}=v$. Conversely, assuming $\mathrm{R} \models u v=v$, we obtain that $\mathrm{R}$ satisfies $u^{n} v=v$ for every positive integer $n$ and therefore $\mathrm{R} \models u^{\omega} v=v$. Assuming further that $c(u)=c(v)$ and invoking Lemma 4.3. we conclude that $\mathrm{R} \models u^{\omega}=u^{\omega} v=v$.

Proposition 6.2 If $\mathrm{R}$ is $\kappa$-reducible for systems consisting of a single word equation without parameters, then $\mathrm{R}$ is completely $\kappa$-reducible.

Proof. In view of the results of Section 3 it remains to show that an equation of $\kappa$-terms $u=v$ can be converted into a finite system of word equations, possibly with word parameters (although we will not require them). We proceed as follows, assuming that $\delta$ is a solution of the equation $u=v$ modulo R. Without loss of generality, we may assume that the constraints specify the content of the value of each variable under a solution modulo $\mathrm{R}$. For each subterm of $u$ or $v$ of the form $t^{\omega}$, we introduce a new variable $x_{t}$ and we add the equation $t^{\prime} x_{t}=x_{t}$ and the constraint $c\left(x_{t}\right)=c(\delta(t))$, where, for a $\kappa$-term $w, w^{\prime}$ is obtained from $w$ by replacing each factor of the form $s^{\omega}$ by the new variable $x_{s}$. Finally, the equation $u=v$ is replaced by the new equation $u^{\prime}=v^{\prime}$. By Lemma 6.1, the solution $\delta$ modulo $\mathrm{R}$ gives rise to a solution $\varepsilon$ of the new system modulo $\mathrm{R}$ by letting it coincide with $\delta$ on the original variables and by taking $\varepsilon\left(x_{t}\right)=\delta\left(t^{\omega}\right)$. On the other hand, if $\bar{\varepsilon}$ is a solution modulo $\mathrm{R}$ in $\kappa$-terms of the new system, then we obtain a solution modulo $\mathrm{R}$ in $\kappa$-terms of the original equation $u=v$ by restricting $\bar{\varepsilon}$ to the original variables.

As an example, consider the single equation of $\kappa$-terms

$$
x z\left((x y)^{\omega} z\right)^{\omega}=y\left((z x)^{\omega} y\right)^{\omega}
$$

with constraints which include the specification of the content of the value of each variable under a solution. Suppose $\delta$ is a solution modulo R. We introduce new variables $\mathrm{a}, \mathrm{b}, \mathrm{c}, \mathrm{d}$ and consider the system consisting of the equations

$$
x y \mathrm{a}=\mathrm{a}, \mathrm{a} z \mathrm{~b}=\mathrm{b}, z x \mathrm{c}=\mathrm{c}, \mathrm{c} y \mathrm{~d}=\mathrm{d}, x z \mathrm{~b}=y \mathrm{~d}
$$

where, to the given constraints on the variables $x, y, z$ we add the following constraints

$$
c(\mathrm{a})=c(\delta(x y)), c(\mathrm{~b})=c(\delta(x y z)), c(\mathrm{c})=c(\delta(x z)), c(\mathrm{~d})=c(\delta(x y z)) .
$$

Then the new system has a solution modulo R. A solution of this system modulo $\mathrm{R}$ in $\kappa$-terms provides a $\kappa$-reduction of the original equation.

To proceed with the simplifications for the $\kappa$-reducibility for R, we introduce the notion of an R-reduced solution. Consider a word equation $u=v$ with clopen constraints. A solution $\delta$ modulo $\mathrm{R}$ of the equation is said to be 
R-reduced with respect to $u=v$ if it has the following property: for every factor $x y$ of $u v$, where $x$ and $y$ are variables, the first letter of $\delta(y)$ does not belong to $\vec{c}(\delta(x))$.

Proposition 6.3 Suppose that $\mathrm{R}$ is $\kappa$-reducible for systems of word equations which involve one equation $u=v$ without parameters and all other equations of the form $x a=x$, where $x$ is a variable and $a \in A$ is a parameter evaluated to itself, and which admit solutions modulo $\mathrm{R}$ which are $\mathrm{R}$-reduced with respect to the equation $u=v$. Then $\mathrm{R}$ is completely $\kappa$-reducible.

Proof. By Proposition 6.2 we know that, to prove that $\mathrm{R}$ is completely $\kappa$ reducible, it suffices to show that $\mathrm{R}$ is $\kappa$-reducible for systems consisting of a single word equation without parameters. Let $u=v$ be an equation with $u, v \in X^{+}$with clopen constraints in $\bar{\Omega}_{A} \mathrm{~S}$. Suppose that $\delta: \bar{\Omega}_{X} \mathrm{~S} \rightarrow \bar{\Omega}_{A} \mathrm{~S}$ is a solution of $u=v$ modulo R. We show how to transform $u=v$ and $\delta$ into a system and solution of the special form described in the statement of the proposition.

For each variable $x \in X$, we consider the first-occurrences factorization of the pseudoword $\delta(x)$ as given by Corollary 4.2](1)

$$
\delta(x)=a_{1, x} \delta(x)_{1} \cdots a_{n_{x}, x} \delta(x)_{n_{x}} .
$$

To describe such a factorization, we consider the extended set of variables

$$
Y=X \uplus\left\{x_{i}: x \in X, 1 \leq i \leq n_{x}\right\},
$$

the set of parameters

$$
P=A \uplus\{\$\},
$$

where each parameter evaluates to itself, and we introduce a new equation

$$
x=a_{1, x} x_{1} \cdots a_{n_{x}, x} x_{n_{x}} .
$$

We impose constraints expressing content conditions on each variable $x_{i}$ :

$$
c\left(x_{i}\right) \subseteq\left\{a_{1, x}, \ldots, a_{i, x}\right\}
$$

while in (6.1) the letters $a_{i, x} \in A$ are parameters. Finally, to capture some information about the cumulative content which will be required later, we consider all equations of the form

$$
x_{n_{x}} a=x_{n_{x}}, \quad a \in \vec{c}\left(\delta(x)_{n_{x}}\right) .
$$

The solution $\delta$ of the original equation, modulo $\mathrm{R}$, gives rise to a continuous homomorphism $\varepsilon: \bar{\Omega}_{Y \cup P} \mathrm{~S} \rightarrow \bar{\Omega}_{P} \mathrm{~S}$ mapping any parameter to itself, and defined on variables by $\varepsilon(x)=\delta(x)$ and $\varepsilon\left(x_{i}\right)=\delta(x)_{i}$ for $x \in X$. Clearly, $\varepsilon$ satisfies the old along with the new constraints. Further, if we apply $\varepsilon$ to 
both sides of each of the equations $u \$=v \$$, (6.1) and (6.3), then we obtain pseudoidentities which are valid in $\mathrm{R}$.

In the equation $u \$=v \$$, we substitute the occurrence of each variable $x$ by its expression given by equation (6.1) and we reduce it by applying the following reduction rule to each side of the new intermediate equation. Let $w x$ be a prefix of either side of the equation, where $x$ is a variable. If $c(\delta(x)) \subseteq \vec{c}(\delta(w))$, then we suppress the indicated occurrence of the variable $x$. Otherwise, we let $i \in\left\{1, \ldots, n_{x}\right\}$ be the minimum index such that $a_{i, x} \notin \vec{c}(\delta(w))$ and we replace the indicated occurrence of $x$ by $a_{i, x} x_{i} \cdots a_{n_{x}, x} x_{n_{x}}$. A new equation $u^{\prime}=v^{\prime}$ is thus obtained by applying the reduction rule to both sides of the equation $u \$=v \$$ until no further application of the rule is possible.

From the above process, we obtain in particular a new system consisting of the equations $u^{\prime}=v^{\prime}$ and (6.1) $(x \in X)$ with the old constraints together with the new constraints (6.2) $\left(x \in X, 1 \leq i \leq n_{x}\right)$ and parameters $a_{i, x}$ and $\$$ interpreted as the corresponding letters of $A \cup\{\$\}$. By applying the simplifications described in Section 3, this new system may be converted into a single word equation, without parameters, and with a solution modulo $\mathrm{R}$ which is R-reduced. We add to this equation the equations (6.3) $(x \in X)$.

A solution $\bar{\varepsilon}$, modulo $\mathrm{R}$, in $\kappa$-terms, of the system thus obtained yields a solution modulo $\mathrm{R}$ in $\kappa$-terms of the original equation simply by restricting $\bar{\varepsilon}$ to $X$. Indeed, the image under $\bar{\varepsilon}$ of the prefix of the right side of (6.1) which was omitted in the reduction procedure to obtain the equation $u^{\prime}=v^{\prime}$ may be inserted without affecting the solution modulo $\mathrm{R}$ because the equations (6.3) together with the evaluation of parameters and (6.2) guarantee that each factor which is inserted has a content which lies in the cumulative content of the prefix to the left of it. By hypothesis, this new system is $\kappa$-reducible for R. Hence so is the original equation.

We end this section with a strengthening of a special case of the $\kappa$ reducibility problem for $\mathrm{R}$ which is implicit but not explicit in [11].

Proposition 6.4 Let $\varphi: \bar{\Omega}_{A} \mathrm{~S} \rightarrow S$ be a continuous homomorphism into a finite semigroup $S$ and let $u_{1}, \ldots, u_{n} \in \bar{\Omega}_{A} \mathrm{~S}$ be pseudowords such that

$$
\mathrm{R} \models u_{1}=\cdots=u_{n} .
$$

Then there exist $\kappa$-terms $w_{1}, \ldots, w_{n}$ such that

(i) $\mathrm{R} \models w_{1}=\cdots=w_{n}$;

(ii) $\varphi\left(u_{i}\right)=\varphi\left(w_{i}\right)(i=1, \ldots, n)$;

(iii) $c\left(u_{i}\right)=c\left(w_{i}\right)(i=1, \ldots, n)$;

(iv) $\vec{c}\left(u_{i}\right)=\vec{c}\left(w_{i}\right)(i=1, \ldots, n)$. 
For shortness, we say that the $n$-tuple $\left(w_{1}, \ldots, w_{n}\right)$ of $\kappa$-terms is a $\kappa$ reduction of $\left(u_{1}, \ldots, u_{n}\right)$ if it satisfies conditions $(i)(i v)$.

Proof. Without loss of generality, we may assume that $S$ has a content function [17, that is that the content function $c: \bar{\Omega}_{A} \mathrm{~S} \rightarrow 2^{A}$ factors through $\varphi$. In this way, property $($ iii $)]$ is subsumed by property [ii)] Note that, by (6.4), we must have $c\left(u_{1}\right)=\cdots=c\left(u_{n}\right)$.

We show by induction on $\left|c\left(u_{1}\right)\right|$ that the $u_{i}$ may be replaced by $\kappa$-terms $w_{i}$ satisfying properties $(i)(i v)$ The case $c\left(u_{1}\right)=\{a\}$ is easy. Indeed, in case $u_{1} \in A^{+}$is a word, then by (6.4) all $u_{i}$ are words and, therefore, they are given by $\kappa$-terms. Otherwise, for each $i, u_{i}=a^{\omega} u_{i}$ and so, choosing a finite power $a^{k_{i}}$ such that $\varphi\left(a^{k_{i}}\right)=\varphi\left(u_{i}\right)$, we may take $w_{i}=a^{\omega+k_{i}}$.

Suppose that the claim holds whenever $\left|c\left(u_{1}\right)\right|<K$ and consider $u_{1}, \ldots, u_{n}$ satisfying (6.4), such that $\left|c\left(u_{1}\right)\right|=K$. Factorize $u_{i}$ as

$$
u_{i}=u_{i, 0} a_{0} \cdot u_{i, 1} a_{1} \cdots u_{i, \ell} a_{\ell} \cdot u_{i, \ell}^{\prime}
$$

where each $a_{p} \in A \backslash c\left(u_{i, p}\right)$ and $c\left(u_{i, 0} a_{0}\right)=c\left(u_{i, 1} a_{1}\right)=\cdots=c\left(u_{i, \ell} a_{\ell}\right) \supseteq$ $c\left(u_{i, \ell}^{\prime}\right)$. In particular, the prefixes $u_{i, 0} a_{0} \cdot u_{i, 1} a_{1} \cdots u_{i, k} a_{k}$ are end-marked pseudowords. By Theorem 4.10 the suprema $\left\|u_{i}\right\|$ of the lengths of such factorizations are the same for all $i$ and, moreover, the sequences of letters $a_{0}, a_{1}, \ldots, a_{\ell}$ are also the same for all $i$, and

$$
\begin{aligned}
& \mathrm{R} \models u_{i, p}=u_{j, p} \quad \text { for all } i, j \\
& \mathrm{R} \models u_{i, \ell}^{\prime}=u_{j, \ell}^{\prime} \quad \text { for all } i, j .
\end{aligned}
$$

By construction, $\left|c\left(u_{i, p}\right)\right|=\left|c\left(u_{1}\right)\right|-1$ and so, by the induction hypothesis and (6.6), for each $p$ there exists a $\kappa$-reduction $\left(w_{1, p}, \ldots, w_{n, p}\right)$ of $\left(u_{1, p}, \ldots, u_{n, p}\right)$.

We next distinguish two cases. In the first case, we assume $\left\|u_{1}\right\|<\infty$. In this case, taking $\ell=\left\|u_{i}\right\|=\left\|u_{1}\right\|$ in (6.5), we also have $\left|c\left(u_{i, \ell}^{\prime}\right)\right|<\left|c\left(u_{1}\right)\right|$. Using (6.7) and applying the induction hypothesis again, we deduce that there exists a $\kappa$-reduction $\left(w_{1, \ell}^{\prime}, \ldots, w_{n, \ell}^{\prime}\right)$ of $\left(u_{1, \ell}^{\prime}, \ldots, u_{n, \ell}^{\prime}\right)$. One may then verify that, taking

$$
w_{i}=w_{i, 0} a_{0} \cdot w_{i, 1} a_{1} \cdots w_{i, \ell} a_{\ell} \cdot w_{i, \ell}^{\prime}
$$

defines a $\kappa$-reduction $\left(w_{1}, \ldots, w_{n}\right)$ of the original $n$-tuple $\left(u_{1}, \ldots, u_{n}\right)$.

In the remaining case, we have $\left\|u_{1}\right\|=\infty$. We then consider, for each $\ell$, the $n$-tuple of elements of $S$

$$
\left(\varphi\left(w_{1,0} a_{0} w_{1,1} a_{1} \cdots w_{1, \ell} a_{\ell}\right), \ldots, \varphi\left(w_{n, 0} a_{0} w_{n, 1} a_{1} \cdots w_{n, \ell} a_{\ell}\right)\right) .
$$

Since $S$ is finite, there are indices $k$ and $\ell$ such that $k<\ell$ and, for $i=$ $1, \ldots, n$,

$$
\begin{aligned}
& \varphi\left(w_{i, 0} a_{0} w_{i, 1} a_{1} \cdots w_{i, k} a_{k}\right)= \\
& \quad=\varphi\left(w_{i, 0} a_{0} w_{i, 1} a_{1} \cdots w_{i, k} a_{k}\left(w_{i, k+1} a_{k+1} \cdots w_{i, \ell} a_{\ell}\right)\right) \\
& \quad=\varphi\left(w_{i, 0} a_{0} w_{i, 1} a_{1} \cdots w_{i, k} a_{k}\left(w_{i, k+1} a_{k+1} \cdots w_{i, \ell} a_{\ell}\right)^{\omega}\right) .
\end{aligned}
$$


Take

$$
w_{i}=w_{i, 0} a_{0} w_{i, 1} a_{1} \cdots w_{i, k} a_{k}\left(w_{i, k+1} a_{k+1} \cdots w_{i, \ell} a_{\ell}\right)^{\omega} w_{i, k}^{\prime}
$$

where here $w_{i, k}^{\prime}$ is chosen to be any word such that $\varphi\left(w_{i, k}^{\prime}\right)=\varphi\left(u_{i, k}^{\prime}\right)(i=$ $1, \ldots, n)$. Then one again verifies that $\left(w_{1}, \ldots, w_{n}\right)$ is a $\kappa$-reduction of the original $n$-tuple $\left(u_{1}, \ldots, u_{n}\right)$.

\section{From systems of equations to systems of bound- ary equations}

We introduce in this section a formalism which is borrowed from Makanin's algorithm for the solution of systems of word equations in the free monoid (cf. [35, Chapter 12]). Since it needs to be suitably adapted, the reader familiar with Makanin's algorithm will find some differences in our approach.

Throughout the remainder of the paper, we fix a continuous homomorphism $\varphi: \bar{\Omega}_{A} S \rightarrow S$ into a finite semigroup $S$.

A system of boundary equations is a tuple $(\mathrm{X}, I, \zeta, \chi$, right, $\mathcal{B})$ where:

- $\mathrm{X}$ is a finite set of variables with a fix-point-free involution $\mathrm{x} \mapsto \overline{\mathrm{x}}$;

- $I$ is a finite set, whose elements are called indices, with a total order $\leq$; we will write $i \prec j$ to mean that $i \leq j$ and there is no $k$ such that $i<k<j$;

- $\zeta:\{(i, j) \in I \times I: i \prec j\} \rightarrow 2^{S}$ is a function;

- $\chi:\{(i, j) \in I \times I: i \prec j\} \rightarrow 2^{A}$ is a function;

- right : $\mathrm{X} \rightarrow I$ is a function;

- $\mathcal{B}$ is a set of quadruples of the form $(i, \mathrm{x}, j, \overline{\mathrm{x}}) \in I \times \mathbf{X} \times I \times \mathbf{X}$, which are called boundary equations, such that, whenever $(i, x, j, \bar{x}) \in \mathcal{B}$, the dual boundary equation $(j, \overline{\mathrm{x}}, i, \mathrm{x})$ also belongs to $\mathcal{B}$.

We extend $\chi$ to a function $\{(i, j) \in I \times I: i \leq j\} \rightarrow 2^{A}$ by letting $\chi(i, j)=$ $\chi\left(j^{-}, j\right)$ where $j^{-} \in I$ is such that $j^{-} \prec j$ in case $i<j$ and we let $\chi(i, i)=\emptyset$. A box of the system is any pair of the form $(i, \mathrm{x})$ such that there exists $j$ for which $(i, \mathrm{x}, j, \overline{\mathrm{x}})$ is a boundary equation. For a variable $\mathrm{x}$, we also define left $(\mathrm{x})$ to be the minimum of the $i \in I$ such that there is a box $(i, \mathrm{x})$, in case there is at least one such box.

A model of the system $(\mathrm{X}, I, \zeta, \chi$, right, $\mathcal{B})$ of boundary equations is a triple $\mathcal{M}=(w, \iota, \Phi)$ where

- $w \in\left(\bar{\Omega}_{A} \mathrm{~S}\right)^{1}$;

- $\iota: I \rightarrow \alpha_{w}+1$ is an order-preserving injection such that, if $I \neq \emptyset$, then $\iota(\max I)=\alpha_{w}$ 
- for each pair $i, j \in I$ with $i \prec j$ and each $s \in \zeta(i, j), \Phi(i, j, s) \in \bar{\Omega}_{A} \mathrm{~S}$.

Before stating the properties which we require from a model, we introduce the following convenient notation: for $i, j \in I$ with $i \leq j$, we set

$$
w(i, j)=w[\iota(i), \iota(j)[.
$$

For instance, consider the pseudoword $w=(a b)^{\omega} a c a^{\omega}$ of Example 4.15] and let $I=\{0,1,2,3\}$ and $\iota=\left(\begin{array}{cccc}0 & 1 & 2 & 3 \\ 0 & \omega & \omega+3 & \omega 2\end{array}\right)$. Then $w(0,1)=(a b)^{\omega} a$, $w(1,2)=c a^{2}$ and $w(2,3)=a^{\omega-2}$.

The following are the properties which we require from the model $\mathcal{M}$ :

(M.1) if $i \prec j$ and $s \in \zeta(i, j)$ then $\varphi(\Phi(i, j, s))=s$;

(M.2) if $i \prec j$ then $\vec{c}(w(i, j))=\chi(i, j)$;

(M.3) if $i \prec j$ and $s \in \zeta(i, j)$ then $\mathrm{R} \models \Phi(i, j, s)=w(i, j)$;

(M.4) if $(i, \mathrm{x}, j, \overline{\mathrm{x}}) \in \mathcal{B}$ then $\mathrm{R} \models w(i, \operatorname{right}(\mathrm{x}))=w(j, \operatorname{right}(\overline{\mathrm{x}}))$.

A system is satisfiable if it has at least one model.

We say that $\mathcal{M}$ is a model in $\kappa$-terms if $w \in \Omega_{A}^{\kappa} S$ and $\Phi$ takes its values in $\Omega_{A}^{\kappa}$ S. It is important to note that, as a consequence of a result from [21, each $w(i, j)$ is then also a $\kappa$-term for every $i, j \in I$ with $i<j$. For future reference, we explicitly state this in the following lemma.

Lemma 7.1 Let $z$ be a pseudoword given by a $\kappa$-term and let $z=z_{1} a z_{2}$ be a factorization such that $z_{1}$ a is end-marked. Then $z_{1}$ and $z_{2}$ are also given by $\kappa$-terms.

We next present a number of properties of systems of boundary equations which admit models and which will be useful later.

Proposition 7.2 Let $\mathcal{S}=(\mathrm{X}, I, \zeta, \chi$, right, $\mathcal{B})$ be a system of boundary equations and suppose that $\mathcal{M}=(w, \iota, \Phi)$ is a model of $\mathcal{S}$. Then the following properties are satisfied:

(S.1) if $(i, \mathrm{x}, j, \overline{\mathrm{x}}) \in \mathcal{B}$ then $i \leq \operatorname{right}(\mathrm{x})$ and $j \leq \operatorname{right}(\overline{\mathrm{x}})$;

(S.2) if $(i, \mathrm{x}, j, \overline{\mathrm{x}}) \in \mathcal{B}$ and $\operatorname{right}(\mathrm{x})>\operatorname{right}(\overline{\mathrm{x}})$ then $i>j$;

(S.3) if $(i, \mathrm{x}, j, \overline{\mathrm{x}}) \in \mathcal{B}$ then $\chi(i, \operatorname{right}(\mathrm{x}))=\chi(j$, right $(\overline{\mathrm{x}}))$;

(S.4) if $(i, \mathrm{x}, j, \overline{\mathrm{x}}),(i, \mathrm{y}, j, \overline{\mathrm{y}}) \in \mathcal{B}$ then

$$
\operatorname{right}(\mathrm{x}) \leq \operatorname{right}(\mathrm{y}) \Longleftrightarrow \operatorname{right}(\overline{\mathrm{x}}) \leq \operatorname{right}(\overline{\mathrm{y}}) .
$$


Proof. Since we have only defined $w(i, j)$ in case $i \leq j$, condition (M.4) implies (S.1). Next, (S.2) follows from Lemma 4.11 together with (M.4) and (S.3) follows from condition (M.2) combined with (M.4),

For (S.4) suppose that $(i, \mathrm{x}, j, \overline{\mathrm{x}}),(i, \mathrm{y}, j, \overline{\mathrm{y}}) \in \mathcal{B}$. By duality, it suffices to prove the implication from left to right in (S.4) Arguing by contradiction, suppose that $\operatorname{right}(x) \leq \operatorname{right}(y)$ and $\operatorname{right}(\bar{x})>\operatorname{right}(\bar{y})$. Then, from the hypothesis that $\iota: I \rightarrow \alpha_{w}+1$ is an order-preserving injection and the definition of $w(p, q)$, we conclude that $w(i, \operatorname{right}(\mathrm{x}))$ is a prefix of $w(i, \operatorname{right}(\mathrm{y}))$ and that $w(j, \operatorname{right}(\overline{\mathrm{y}}))$ is a proper prefix of $w(j, \operatorname{right}(\overline{\mathrm{x}}))$. On the other hand, by (M.4) R satisfies the pseudoidentities $w(i, \operatorname{right}(\mathrm{x}))=w(j$, right $(\overline{\mathrm{x}}))$ and $w(i, \operatorname{right}(\mathrm{y}))=w(j, \operatorname{right}(\overline{\mathrm{y}}))$. This leads to the following relations:

$$
\begin{aligned}
& w(i, \operatorname{right}(\mathrm{x})) \geq_{\mathcal{R}} w(i, \operatorname{right}(\mathrm{y}))={ }_{\mathrm{R}} w(j, \operatorname{right}(\overline{\mathrm{y}})) \\
& w(j, \operatorname{right}(\overline{\mathrm{y}})) \geq_{\mathcal{R}} w(j, \operatorname{right}(\overline{\mathrm{x}}))={ }_{\mathrm{R}} w(i, \operatorname{right}(\mathbf{x})) .
\end{aligned}
$$

Hence $\mathrm{R}$ satisfies $w(j, \operatorname{right}(\overline{\mathrm{y}}))=w(j, \operatorname{right}(\overline{\mathrm{x}}))$, which contradicts the assumption that the pseudoword $w(j, \operatorname{right}(\overline{\mathrm{y}}))$ is a proper prefix of $w(j, \operatorname{right}(\overline{\mathrm{x}}))$ by Corollary 4.14 .

We use the same visual notation as in [35, Chapter 12] for representing boxes and boundary equations. From Proposition [7.2 (S.1) every box $(i, \mathrm{x})$ of a satisfiable system of boundary equations is such that $i \leq \operatorname{right}(\mathrm{x})$. We represent such a box by the following picture:

$$
\begin{array}{ll|}
i & \mathrm{x} \\
\hline
\end{array}
$$

The box starts at index $i$ and ends at index $\operatorname{right}(\mathrm{x})$. We say that the right value of the box $(i, \mathrm{x})$ is right $(\mathrm{x})$. The relative orders of beginnings and ends of boxes can be read on such pictures.

For instance, Proposition 7.2 (S.4) states that the situation of Figure 1 $(a)$ cannot occur in a satisfiable system. Similarly, by Proposition 7.2 (S.2) a boundary equation $(i, \mathrm{x}, j, \overline{\mathrm{x}})$ such that $j \leq i$ and $\operatorname{right}(\mathrm{x})<\operatorname{right}(\overline{\mathrm{x}})$, as pictured in Figure $1(b)$ and $\left(b^{\prime}\right)$, cannot occur either in a satisfiable system.

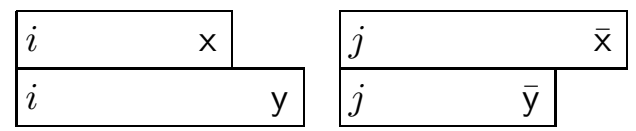

(a)

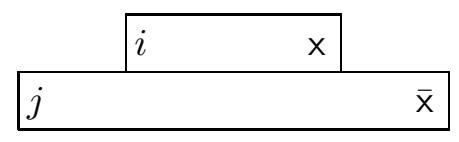

(b)

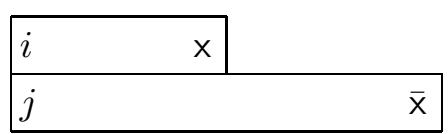

$\left(b^{\prime}\right)$

Figure 1: Some forbidden configurations for a satisfiable system.

In the proof of Makanin's algorithm, a boundary equation $(i, \mathrm{x}, j, \overline{\mathrm{x}})$ of a satisfiable system with $\operatorname{right}(\mathrm{x})=\operatorname{right}(\overline{\mathrm{x}})$ must have its two boxes aligned, 
that is, $i=j$, as in Figure 2. This is because $(i, \mathrm{x})$ and $(j, \overline{\mathrm{x}})$ represent equal factors of the solution ending at the same position $\operatorname{right}(x)=\operatorname{right}(\bar{x})$.

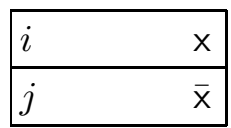

Figure 2: A boundary equation whose boxes are aligned.

In our framework, this condition is replaced by (M.4), which allows boxes of "different lengths" ending, but not starting, at the same position. Such a boundary equation will be called elastic.

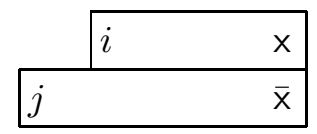

Figure 3: An elastic boundary equation.

To prove the complete reducibility of $\mathrm{R}$, in view of Proposition 6.3 it suffices to consider a system $\mathcal{S}$ consisting of an equation $u=v$, with $u, v \in$ $X^{+}$, together with the equations $x a=x$, where $a \in A_{x}$ and $x \in X$, for a family $\left(A_{x}\right)_{x \in X}$ of subsets of $A$, which express that, in a solution, the cumulative content of the value of $x$ contains $A_{x}$. Here the letters $a$ may be viewed as parameters since we are going to treat separately the equations $x a=x$. Constraints are given by the fixed continuous homomorphism $\varphi$ : $\bar{\Omega}_{A} \mathrm{~S} \rightarrow S$ and a family $\left(s_{x}\right)_{x \in X}$ of elements of $S$. We also suppose that a solution $\delta: \bar{\Omega}_{X} S \rightarrow \bar{\Omega}_{A} S$, modulo $\mathrm{R}$, of the system $\mathcal{S}$ is given which, furthermore, is R-reduced for the equation $u=v$.

We associate with such a system $\mathcal{S}$, together with a solution, a system of boundary equations

$$
\mathcal{S}^{\prime}=(\mathrm{X}, I, \zeta, \chi, \text { right }, \mathcal{B}) .
$$

The construction is borrowed from Makanin's algorithm. Its purpose is to organize the matching of both factorizations by only matching a pair of factorizations of the same word at a time.

Example 7.3 Let us first illustrate the construction with an example. Consider the equation $x y z x=y z x t y$. Then, the matching might be done as indicated in the following diagram:

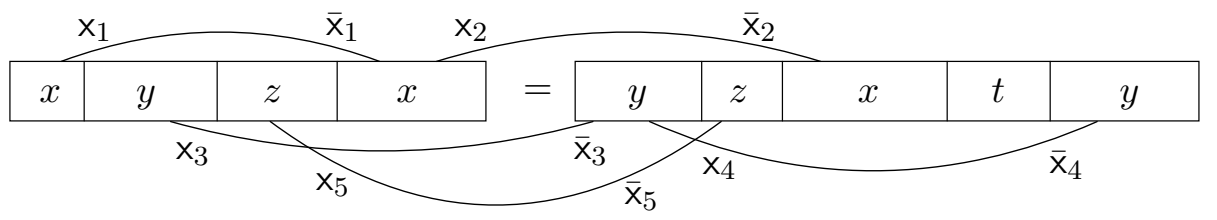


The new variables $\mathrm{x}_{1}$ to $\mathrm{x}_{5}$ of the system of boundary equations can be seen as pairs $(p, q)$, where $p$ and $q$ represent positions of two occurrences of the same original variable in the word $u v$, where $u=v$ is the equation we started with, in our example $u=x y z x$ and $v=y z x t y$. For instance, since $x$ occurs at positions 1, 4 and 7 in the product $x y z x \cdot y z x t y$ of both words defining the original equation, we introduce two variables $\mathrm{x}_{1}=(1,4)$ and $\mathrm{x}_{2}=(4,7)$. In the translation from systems of equations to systems of boundary equations, the purpose of a new variable $(p, q)$ is to capture information that, in the original equation, factors of $\delta(u v)$ corresponding to the value of two occurrences of the same variable $x$, at positions $p$ and $q$, are equal (to $\delta(x)$ ). Introducing another variable $(1,7)$ is useless, since the relationship between the corresponding factors of the solution is already captured by the previous variables, by transitivity of equality.

In the system $\mathcal{S}^{\prime}$ of boundary equations, there are two additional variables, $\mathrm{x}_{0}$ and $\overline{\mathrm{x}}_{0}$, used to match the common value of both sides of the equation, over $\mathrm{R}$ (but not necessarily over $\mathrm{S}$ ). Each box is then identified by the position $i$ of its beginning (its left) together with the new variable $\mathrm{x}_{k}$ or $\overline{\mathrm{x}}_{k}$ that determines it. In the example, we obtain the following representation.

\begin{tabular}{|c|c|c|c|c|c|c|c|c|c|}
\hline$i_{0}$ & & & $\mathrm{x}_{0}$ & & & & & & $\overline{\mathrm{x}}_{0}$ \\
\hline \begin{tabular}{ll|l}
$i_{0}$ & $\mathrm{x}_{1}$ & $i_{1}$
\end{tabular} & $\begin{array}{ll}\mathrm{x}_{3} & i_{2}\end{array}$ & $\mathrm{x}_{5} \mid$ & $x_{2}$ & $i_{4}$ & $x_{4}$ & \begin{tabular}{ll|l}
$i_{5}$ & $\bar{x}_{5}$ & $i_{6}$
\end{tabular} & $\bar{x}_{2}$ & $i_{7}$ & $\bar{x}_{4}$ \\
\hline & & $i$ & $\overline{\mathrm{x}}_{1}$ & $i_{4}$ & $\bar{x}_{3}$ & & & & \\
\hline
\end{tabular}

This representation defines the variables, boundary equations and the value of the right function. The remaining parts of the system of boundary equations $(I, \zeta$, and $\chi)$ will be defined in terms of the relevant values of the corresponding factors of the solution (associated ordinal, value over the semigroup $S$, cumulative content). From the solution of the original equation system $\mathcal{S}$, we shall also derive a model of the system of boundary equations.

Let us formalize the construction illustrated in Example 7.3 First, we let $u=x_{1} \cdots x_{r}$ and $v=x_{r+1} \cdots x_{s}$, where the $x_{p} \in X$, and we consider the undirected graph with vertex set $\{1, \ldots, s\}$ which has an edge $\{p, q\}$ if and only if $x_{p}=x_{q}$ and $p \neq q$. Let $i_{0}=0$ and $i_{p}=\alpha_{\delta\left(x_{1} \cdots x_{p}\right)}$ for $p=1, \ldots, s$. Next, we choose a spanning forest $F$ for this graph. We then set the defining components of our system of boundary equations as follows:

- we put in $\mathrm{X}$ all pairs $(p, q)$ such that $\{p, q\} \in F$ and we let $\overline{(p, q)}=(q, p)$;

- $I=\left\{i_{0}, \ldots, i_{s}\right\}$ ordered by the usual order between ordinals;

- $\zeta\left(i_{p-1}, i_{p}\right)=\left\{\varphi\left(\delta\left(x_{p}\right)\right)\right\}=\left\{s_{x_{p}}\right\}$;

- $\chi\left(i_{p-1}, i_{p}\right)$ is the set $\vec{c}\left(\delta\left(x_{p}\right)\right)$, and therefore it contains $A_{x_{p}}$; 
- for $(p, q) \in \mathrm{X}$, we let $\operatorname{right}(p, q)=i_{p}$;

- for each $(p, q) \in \mathbf{X}$, we put in $\mathcal{B}$ the boundary equation

$$
\left(i_{p-1},(p, q), i_{q-1},(q, p)\right)
$$

- we add to $X$ a pair of variables $I, r$ with $\bar{I}=r$ and $\bar{r}=I$, and we set $\operatorname{right}(\mathrm{I})=i_{r}$ and $\operatorname{right}(\mathrm{r})=i_{s}$;

- we add to $\mathcal{B}$ the boundary equations $\left(i_{0}, \mathrm{I}, i_{r}, \mathrm{r}\right)$ and $\left(i_{r}, \mathrm{r}, i_{0}, \mathrm{I}\right)$.

We also define a candidate for a model of the system of boundary equations $\mathcal{S}^{\prime}$ which is given by $\mathcal{M}_{\mathcal{S}}=(w, \iota, \Phi)$, where:

- $w$ is the pseudoword $\delta(u v)$;

- $\iota$ is the inclusion mapping of $I$ into the ordinal $\alpha_{w}+1$;

- for each $p \in\{1, \ldots, s\}, \Phi\left(i_{p-1}, i_{p}, s_{x_{p}}\right)=w\left(i_{p-1}, i_{p}\right)=\delta\left(x_{p}\right)$.

Proposition 7.4 The tuple $\mathcal{S}^{\prime}$ given by (7.1) is a system of boundary equations and $\mathcal{M}_{\mathcal{S}}$ is a model of $\mathcal{S}^{\prime}$. Moreover, if the system of boundary equations $\mathcal{S}^{\prime}$ admits a model in $\kappa$-terms, then the original system $\mathcal{S}$ has a solution, modulo $\mathrm{R}$, in $\kappa$-terms.

Proof. To verify that $\mathcal{M}_{\mathcal{S}}$ is a model of $\mathcal{S}^{\prime}$, we observe that conditions (M.1) (M.2) and (M.3) are given directly by the definition of $\zeta, \chi$, and $\Phi$. If $(i, \mathrm{x}, j, \overline{\mathrm{x}})$ is a boundary equation, then the pseudoidentity

$$
w(i, \operatorname{right}(\mathbf{x}))=w(j, \operatorname{right}(\overline{\mathbf{x}}))
$$

is trivial unless $\mathrm{x}$ is one of the variables I or $\mathrm{r}$, in which case the above pseudoidentity is valid in $\mathrm{R}$ since $\delta(u)=\delta(v)$. This proves condition (M.4)

Finally, assume that $\left(w^{\prime}, \iota^{\prime}, \Phi^{\prime}\right)$ is a model in $\kappa$-terms of $\mathcal{S}^{\prime}$. For each variable $x \in X$ which occurs in the equation $u=v$, choose any $p \in\{1, \ldots, s\}$ such that $x_{p}=x$. Let

$$
\varepsilon(x)=\Phi^{\prime}\left(i_{p-1}, i_{p}, s_{x_{p}}\right) .
$$

We extend $\varepsilon$ to all of $X$ by choosing, for each variable $x^{\prime} \in X$ which does not occur in $u=v$, a suitable $\kappa$-term $\varepsilon\left(x^{\prime}\right)$ such that $\varphi\left(\varepsilon\left(x^{\prime}\right)\right)=s_{x^{\prime}}$ and $\vec{c}\left(\varepsilon\left(x^{\prime}\right)\right) \supseteq A_{x^{\prime}}$, whose existence is guaranteed by Proposition [6.4. By condition (M.1) the constraints of the original system $\mathcal{S}$ are satisfied by $\varepsilon$. By condition (M.2) and the definition of $\varepsilon$ on variables which do not occur in $u=v$, the equations $x a=x$ of the system $\mathcal{S}$ are satisfied, modulo $\mathrm{R}$, by $\varepsilon$. By the case of condition (M.4) corresponding to the boundary equation $\left(i_{0}, \mathrm{l}, i_{r}, \mathrm{r}\right)$, we obtain that

$$
\mathrm{R} \models w^{\prime}\left(i_{0}, i_{r}\right)=w^{\prime}\left(i_{r}, i_{s}\right)
$$


By definition of $w^{\prime}(i, j)$, we have the factorizations

$$
\begin{aligned}
& w^{\prime}\left(i_{0}, i_{r}\right)=w^{\prime}\left(i_{0}, i_{1}\right) \cdots w^{\prime}\left(i_{r-1}, i_{r}\right) \\
& w^{\prime}\left(i_{r}, i_{s}\right)=w^{\prime}\left(i_{r}, i_{r+1}\right) \cdots w^{\prime}\left(i_{s-1}, i_{s}\right) .
\end{aligned}
$$

By condition (M.3), we have

$$
\mathrm{R} \models w^{\prime}\left(i_{p-1}, i_{p}\right)=\Phi^{\prime}\left(i_{p-1}, i_{p}, s_{x_{p}}\right) .
$$

Combining (7.2), (7.3), (7.4), and (7.5), we conclude that $\varepsilon$ is a solution modulo $\mathrm{R}$ of the equation $u=v$. Hence $\mathcal{S}$ has a solution modulo $\mathrm{R}$ in $\kappa$-terms.

Let us emphasize that, given a model $(w, \iota, \Phi)$ of a system of boundary equations containing the boundary equation $(i, \mathrm{x}, j, \overline{\mathrm{x}})$, we do not require that $w(i, \operatorname{right}(\mathbf{x}))$ and $w(j, \operatorname{right}(\overline{\mathrm{x}}))$ be equal, even if this happens to be the case for the boundary equation system $\mathcal{S}^{\prime}$ obtained from $\mathcal{S}$ (except for $\mathrm{x}=\mathrm{x}_{0}$ ). Instead, condition (M.4) just imposes these pseudowords to project on the same element of $\bar{\Omega}_{A} \mathrm{R}$. It turns out that, for $\mathcal{S}^{\prime}$, these conditions are sufficient to recover, from a model of the system of boundary equations, a solution of the original equation.

Another remark is that the boundary equation system $\mathcal{S}^{\prime}$ we obtain from an equation system $\mathcal{S}$ is such that $|\zeta(i, j)|=1$ for $i \prec j$. This again is very special and more general systems, where $|\zeta(i, j)|>1$, will come up during the forthcoming constructions.

Combining Proposition 7.4 with Proposition 6.3, we obtain yet another sufficient condition for the complete $\kappa$-reducibility of R.

Corollary 7.5 If every system of boundary equations which has a model also has a model in $\kappa$-terms, then $\mathrm{R}$ is completely $\kappa$-reducible.

\section{Factorization schemes and refinements}

We gather in this section a couple of technical results which will be used repeatedly in the next section.

Let $w \in \bar{\Omega}_{A} \mathrm{~S}$. By a factorization scheme for $w$ we mean a triple $\mathcal{C}=$ $(I, \iota, \Phi)$ where:

- $I$ is a finite totally ordered set for which we use the same notation as in the definition of system of boundary equations;

- $\iota: I \rightarrow \alpha_{w}+1$ is an order preserving injective function;

- $\Phi$ is a partial function whose domain is contained in the set $\{(i, j, s) \in$ $I \times I \times S: i \prec j\}$ and which takes its values in $\bar{\Omega}_{A} \mathrm{~S}$. 
We further require that the following pointlike properties be satisfied by $\mathcal{C}$ whenever the pseudoword $\Phi(i, j, s)$ is defined:

$($ PL.1) $\mathrm{R} \models \Phi(i, j, s)=w[\iota(i), \iota(j)[$;

(PL.2) $\varphi(\Phi(i, j, s))=s$.

Given two factorization schemes for the pseudoword $w, \mathcal{C}_{1}=\left(I_{1}, \iota_{1}, \Phi_{1}\right)$ and $\mathcal{C}_{2}=\left(I_{2}, \iota_{2}, \Phi_{2}\right)$, we say that $\mathcal{C}_{1}$ refines $\mathcal{C}_{2}$ if the following conditions are satisfied:

(R.1) $\operatorname{Im} \iota_{2} \subseteq \operatorname{Im} \iota_{1}$

(R.2) if $i \prec j$ in $I_{2}, i_{0} \prec i_{1} \prec \cdots \prec i_{n}$ in $I_{1}, \iota_{2}(i)=\iota_{1}\left(i_{0}\right), \iota_{2}(j)=\iota_{1}\left(i_{n}\right)$, and $\Phi_{2}(i, j, s)$ is defined, then there exists in $S$ a factorization $s=s_{1} \cdots s_{n}$ such that each $\Phi_{1}\left(i_{m-1}, i_{m}, s_{m}\right)$ is defined $(m=1, \ldots, n)$.

Notice that, in particular, for each element $i_{2} \in I_{2}$ there exists a unique element $i_{1} \in I_{1}$, such that $\iota_{1}\left(i_{1}\right)=\iota_{2}\left(i_{2}\right)$, namely $i_{1}=\iota_{1}^{-1}\left(\iota_{2}\left(i_{2}\right)\right)$. Therefore, when convenient, we will regard $I_{2}$ as a subset of $I_{1}$, following the convention of identifying each $i_{2} \in I_{2}$ with $\iota_{1}^{-1}\left(\iota_{2}\left(i_{2}\right)\right) \in I_{1}$.

Proposition 8.1 Let $\mathcal{C}_{1}=\left(I_{1}, \iota_{1}, \Phi_{1}\right)$ and $\mathcal{C}_{2}=\left(I_{2}, \iota_{2}, \Phi_{2}\right)$ be factorization schemes for the pseudoword $w$. Then there exists a common refinement $\mathcal{C}_{3}=\left(I_{3}, \iota_{3}, \Phi_{3}\right)$ of $\mathcal{C}_{1}$ and $\mathcal{C}_{2}$ such that $\operatorname{Im} \iota_{3}=\operatorname{Im} \iota_{1} \cup \operatorname{Im} \iota_{2}$. Moreover, if all the $\Phi_{1}(i, j, s)$ and $\Phi_{2}(i, j, s)$ which are defined are given by $\kappa$-terms, then the same property holds for $\Phi_{3}$.

Proof. Let $I_{3}=\operatorname{Im} \iota_{1} \cup \operatorname{Im} \iota_{2}$ and let $\iota_{3}: I_{3} \hookrightarrow \alpha_{w}+1$ be the inclusion mapping. We start by setting $\Phi_{3}(\beta, \gamma, s)$ to be undefined for all $\beta, \gamma \in I_{3}$ such that $\beta \prec \gamma$ in $I_{3}$ and all $s \in S$. We will describe a situation that may provide candidates for the definition of $\Phi_{3}(\beta, \gamma, s)$ and, since any of those candidates will do the job, we just choose one of them.

Suppose that $k \in\{1,2\}, i, j \in I_{k}$, and $s \in S$ are such that $i \prec j$ and $\Phi_{k}(i, j, s)$ is defined. Let $\ell$ be the element of $\{1,2\} \backslash\{k\}$. Suppose further that the inverse image of the interval $\left[\iota_{k}(i), \iota_{k}(j)\right]$ under $\iota_{\ell}$ consists of the indices $p_{1}, \ldots, p_{n} \in I_{\ell}$ such that $p_{1} \prec \cdots \prec p_{n}$. We let $\beta_{0}, \ldots, \beta_{t}$ be the elements of the set $\left\{\iota_{k}(i), \iota_{\ell}\left(p_{1}\right), \ldots, \iota_{\ell}\left(p_{n}\right), \iota_{k}(j)\right\}$, so that $\beta_{0}<\cdots<\beta_{t}$. By Corollary 4.14 and (PL.1) for $\mathcal{C}_{k}$, there is a unique factorization $\Phi_{k}(i, j, s)=$ $z_{1} \cdots z_{t}$ such that $\mathrm{R} \models z_{m}=w\left[\beta_{m-1}, \beta_{m}\left[\right.\right.$ for $m=1, \ldots, t$. Then $z_{m}$ is one of the candidates for the definition of $\Phi_{3}\left(\beta_{m-1}, \beta_{m}, \varphi\left(z_{m}\right)\right)$.

It remains to check that $\mathcal{C}_{3}=\left(I_{3}, \iota_{3}, \Phi_{3}\right)$ is a factorization scheme for $w$ which is a common refinement of $\mathcal{C}_{1}$ and $\mathcal{C}_{2}$. The properties (PL.1) and (PL.2) are immediate by construction: if $\Phi_{3}(\beta, \gamma, s)$ has been defined then it has been set to be a pseudoword $z$ such that $\varphi(z)=s$ and $\mathrm{R} \models z=w[\beta, \gamma[$.

Condition (R.1) is immediate from the choice of $I_{3}$ and $\iota_{3}$. For condition (R.2) consider $k \in\{1,2\}$ and $i, j \in I_{k}$ such that $i \prec j$ in $I_{k}$. Suppose 
that $\Phi_{k}(i, j, s)$ is defined. Let $\beta_{0}, \ldots, \beta_{n} \in I_{3}$ be such that $\beta_{0}=\iota_{k}(i)$, $\beta_{n}=\iota_{k}(j)$, and $\beta_{0} \prec \beta_{1} \prec \cdots \prec \beta_{n}$ in $I_{3}$. Since $i \prec j$ in $I_{k}$, if $n>1$ then the ordinals $\beta_{1}, \ldots, \beta_{n-1}$ belong to $\operatorname{Im} \iota_{\ell}$, where $\{k, \ell\}=\{1,2\}$. The ordinals $\beta_{0}$ and $\beta_{n}$ may or may not belong to $\operatorname{Im} \iota_{\ell}$. In any case, if both $\iota_{\ell}^{-1}\left(\beta_{m-1}\right)$ and $\iota_{\ell}^{-1}\left(\beta_{m}\right)$ are defined then $\iota_{\ell}^{-1}\left(\beta_{m-1}\right) \prec \iota_{\ell}^{-1}\left(\beta_{m}\right)$ in $I_{\ell}$. Then, by the definition of $\Phi_{3}$, the unique factorization $\Phi_{k}(i, j, s)=z_{1} \cdots z_{n}$ such that $\mathrm{R} \models z_{m}=w\left[\beta_{m-1}, \beta_{m}\right.$ [ gives rise to a candidate $z_{m}$ for the definition of $\Phi_{3}\left(\beta_{m-1}, \beta_{m}, \varphi\left(z_{m}\right)\right)$. Even if this is not the candidate that has been chosen for that definition, all that condition (R.2) requires is that each pseudoword $\Phi_{3}\left(\beta_{m-1}, \beta_{m}, \varphi\left(z_{m}\right)\right)$ be defined, and this is certainly guaranteed. The last assertion from the proposition follows directly from Lemma 7.1. $\square$

We also have the following easier result whose proof amounts to a straightforward verification of the required properties.

Proposition 8.2 Suppose that $\mathcal{C}_{1}=\left(I_{1}, \iota_{1}, \Phi_{1}\right)$ is a factorization scheme for $w$ and let $\iota_{2}: I_{2} \rightarrow \alpha_{w}+1$ be an order preserving injection of another totally ordered set $I_{2}$ into $\alpha_{w}+1$ such that $\iota_{2}\left(I_{2}\right) \subseteq \iota_{1}\left(I_{1}\right)$. Define $\mathcal{C}_{2}=$ $\left(I_{2}, \iota_{2}, \Phi_{2}\right)$, where

- if $i_{0} \prec \cdots \prec i_{n}$ in $I_{1}, i=\iota_{2}^{-1}\left(\iota_{1}\left(i_{0}\right)\right), j=\iota_{2}^{-1}\left(\iota_{1}\left(i_{n}\right)\right), i \prec j$ in $I_{2}$, and each $\Phi_{1}\left(i_{m-1}, i_{m}, s_{m}\right)$ is defined, then we take the product $\prod_{m=1}^{n} \Phi_{1}\left(i_{m-1}, i_{m}, s_{m}\right)$ to be one of the candidates for the definition of $\Phi_{2}\left(i, j, \prod_{m=1}^{n} s_{m}\right)$;

- in case there is at least one candidate for the definition of $\Phi_{2}(i, j, s)$ then we choose any such candidate to define $\Phi_{2}(i, j, s)$.

Then $\mathcal{C}_{2}$ is a factorization scheme for $w$ and $\mathcal{C}_{1}$ is a refinement of $\mathcal{C}_{2}$. If all the $\Phi_{1}(i, j, s)$ which are defined are given by $\kappa$-terms, then the similar property holds for $\Phi_{2}$.

A factorization scheme $\mathcal{C}_{2}$ as given by Proposition 8.2 is said to be a restriction of $\mathcal{C}_{1}$ to $I_{2}$.

\section{Systems of boundary equations}

To achieve our programme for the proof of complete $\kappa$-reducibility of R, it remains to show that any system of boundary equations which admits a model also admits a model in $\kappa$-terms (cf. Corollary [7.5). This will be established by transfinite induction on a suitable parameter. For a system $\mathcal{S}=(\mathbf{X}, I, \zeta, \chi$, right, $\mathcal{B})$ and a model $\mathcal{N}=(w, \iota, \Phi)$ of $\mathcal{S}$, the parameter in question is

$$
[\mathcal{S}, \mathcal{M}]=\left(\alpha_{w}, n\right)
$$

where $n$ is the number of boxes $(i, \mathrm{x})$ in the system $\mathcal{S}$ such that right $(\mathrm{x})=$ $\max I$. The pairs of the form $(\alpha, n)$, where $\alpha$ is an ordinal and $n$ is a nonnegative integer, are ordered lexicographically so that $(\alpha, n) \leq(\beta, m)$ if and only if either $\alpha<\beta$, or $\alpha=\beta$ and $n \leq m$. 
The induction hypothesis is that, for every system $\mathcal{S}_{1}$ of boundary equations and model $\mathcal{M}_{1}$ of $\mathcal{S}_{1}$ such that $\left[\mathcal{S}_{1}, \mathcal{M}_{1}\right]<[\mathcal{S}, \mathcal{M}], \mathcal{S}_{1}$ admits a model in $\kappa$-terms. We proceed to show that $\mathcal{S}$ also admits a model in $\kappa$-terms. If $|I| \leq 1$ then, for the appropriate function $\imath,(w, \imath, \emptyset)$ is a model for every $\kappa$-term $w$, and so we may assume that $|I|>1$, and in particular $I \neq \emptyset$.

The induction step consists in associating with the pair $(\mathcal{S}, \mathcal{M})$ a new pair $\left(\mathcal{S}_{1}, \mathcal{M}_{1}\right)$ such that $\mathcal{S}_{1}$ is a system of boundary equations and $\mathcal{M}_{1}$ is a model of $\mathcal{S}_{1}$ and the following properties are satisfied:

(P.1) $\left[\mathcal{S}_{1}, \mathcal{M}_{1}\right]<[\mathcal{S}, \mathcal{M}]$;

(P.2) if there is a model of $\mathcal{S}_{1}$ in $\kappa$-terms, then there is also a model of $\mathcal{S}$ in $\kappa$-terms.

The induction step is subdivided into several cases, according to the pattern formed by boundary equations whose right value of one of their associated boxes is maximal in $I$. It will be described following, as much as possible, the rules in Makanin's algorithm. Yet, unlike what happens in Makanin's algorithm, we may well have a boundary equation $(i, \mathrm{x}, j, \overline{\mathrm{x}})$ with $i \neq j$ and $\operatorname{right}(\mathrm{x})=\operatorname{right}(\overline{\mathrm{x}})$ (see Figure 3). Recall that such a boundary equation is said to be elastic. We will need an auxiliary step to handle elastic boundary equations which will also involve constructing a pair $\left(\mathcal{S}_{(1)}, \mathcal{M}_{(1)}\right)$ from $(\mathcal{S}, \mathcal{M})$ but without changing the induction parameter. It will be convenient to describe this step only after considering some of the cases of the induction step.

Here is an informal overview of which situation each case handles. We assume globally that whenever we enter one of the following cases, all preceding cases do not apply.

Case 1. There is a variable with only empty associated boxes, at the right end of the interval $I$.

Case 2. There is a boundary equation $(i, \mathrm{x}, i, \overline{\mathrm{x}})$ whose aligned boxes (see Figure 2), end at $\max I$.

Case 3. There is no box ending at $\max I$.

Case 4. There is an elastic boundary equation $(i, \mathrm{x}, j, \overline{\mathrm{x}})$ whose boxes end at $\max I$, with $i<j$ and $c(w(i, j)) \varsubsetneqq c(w(i, \operatorname{right}(\mathrm{x})))$.

Case 5. There is a boundary equation which is not elastic, one of whose boxes ends at max $I$.

Case 6. There is an elastic boundary equation ending at $\max I$, and Case 4 does not apply. 
These cases cover all possibilities in view of Proposition 7.2. We now prove that, for each of them, one can derive from the system $\mathcal{S}$ and its model $\mathcal{M}$ a system $\mathcal{S}_{1}$ and a model $\mathcal{M}_{1}$ of $\mathcal{S}_{1}$, satisfying (P.1) and (P.2) In each case, if all boxes involving a variable are removed from the system, then it is understood that we also remove that variable.

The first three cases are easy. They handle situations where one can remove from the system boundary equations without modifying the model (Cases 1 and 2), or shrink the set $I$ (as well as the solution) because its maximal element is not a value under the right function (Case 3).

Case 1. If there is a variable $\mathrm{x}$ such that $\operatorname{left}(\mathrm{x})=\operatorname{right}(\mathrm{x})=\max I$, then delete the boundary equations involving $x$ and $\bar{x}$. This leads to a new system $\mathcal{S}_{1}$ which has exactly the same models as $\mathcal{S}$ and it is such that $\left[\mathcal{S}_{1}, \mathcal{M}\right]<$ $[\mathcal{S}, \mathcal{M}]$. Hence properties (P.1) and (P.2) are verified and the induction step is achieved in this case.

Case 2. If there is any boundary equation $(i, \mathrm{x}, i, \overline{\mathrm{x}})$ such that $\operatorname{right}(\mathrm{x})=$ $\operatorname{right}(\overline{\mathrm{x}})=\max I$, then both boxes $(i, \mathrm{x})$ and $(i, \overline{\mathrm{x}})$ represent the same factor of the solution. In this case, we delete the boundary equations $(i, \mathrm{x}, i, \overline{\mathrm{x}})$ and $(i, \overline{\mathrm{x}}, i, \mathrm{x})$ to obtain a new system $\mathcal{S}_{1}$. The induction step is achieved here precisely for the same reasons as in Case 1 .

Case 3. If there is no box $(i, \mathrm{x})$ such that $\operatorname{right}(\mathrm{x})=\max I$, then we let $J=I \backslash\{\max I\}$, and we define a new system

$$
\mathcal{S}_{1}=\left(\mathrm{X}, J,\left.\zeta\right|_{J \times J},\left.\chi\right|_{J \times J}, \text { right, } \mathcal{B}\right) .
$$

Let $r=\max I$ and let $c=\max J$. Let $w_{1}=w[0, \iota(c)[$. For $i, j \in J$ with $i \prec j$ and $s \in \zeta(i, j)$, let $\Phi_{1}(i, j, s)=\Phi(i, j, s)$. We observe that $\mathcal{M}_{1}=\left(w_{1},\left.\iota\right|_{J}, \Phi_{1}\right)$ is a model of $\mathcal{S}_{1}$ such that $\alpha_{w_{1}}=\iota(c)<\alpha_{w}$, which guarantees the property (P.1)] To establish property (P.2)] suppose that $\mathcal{M}_{1}^{\prime}=\left(w_{1}^{\prime} z^{\prime}, \iota_{1}^{\prime}, \Phi_{1}^{\prime}\right)$ is a model of $\mathcal{S}_{1}$ in $\kappa$-terms such that $\iota_{1}^{\prime}(c)=\alpha_{w_{1}^{\prime}}$ and, if $z^{\prime} \neq 1$ then the first letter of $z^{\prime}$ is not in $\vec{c}\left(w_{1}^{\prime}\right)$. By Lemma 7.1 both $w_{1}^{\prime}$ and $z^{\prime}$ are $\kappa$-terms.

If $\zeta(c, r)=\emptyset$, then we let $w_{2}^{\prime}$ be any $\kappa$-term such that $\vec{c}\left(w_{2}^{\prime}\right)=\chi(c, r)$. In the case where $\zeta(c, r) \neq \emptyset$, since by condition (M.3) we have $\mathrm{R} \models \Phi\left(c, r, s_{1}\right)=$ $w(c, r)=\Phi\left(c, r, s_{2}\right)$ whenever $s_{1}, s_{2} \in \zeta(c, r)$, we deduce from Proposition 6.4 that, for each $s \in \zeta(c, r)$, there exists a $\kappa$-term $\Phi^{\prime}(c, r, s)$ such that

- $\varphi\left(\Phi^{\prime}(c, r, s)\right)=\varphi(\Phi(c, r, s))$ whenever $s \in \zeta(c, r)$;

- $\vec{c}\left(\Phi^{\prime}(c, r, s)\right)=\vec{c}(\Phi(c, r, s))$ whenever $s \in \zeta(c, r)$;

- $\mathrm{R} \models \Phi^{\prime}\left(c, r, s_{1}\right)=\Phi^{\prime}\left(c, r, s_{2}\right)$ whenever $s_{1}, s_{2} \in \zeta(c, r)$.

We then choose any $s_{0} \in \zeta(c, r)$ and we let $w_{2}^{\prime}=\Phi^{\prime}\left(c, r, s_{0}\right)$. For $i, j \in J$ with $i \prec j$ and $s \in \zeta(i, j)$, we set $\Phi^{\prime}(i, j, s)=\Phi_{1}^{\prime}(i, j, s)$. We take $w^{\prime}=w_{1}^{\prime} w_{2}^{\prime}$. Finally, we set $\mathcal{M}^{\prime}=\left(w^{\prime}, \iota^{\prime}, \Phi^{\prime}\right)$, where $\iota^{\prime}$ extends $\iota_{1}^{\prime}$ by letting $\iota^{\prime}(r)=\alpha_{w^{\prime}}$. It 
is then easy to verify that $\mathcal{M}^{\prime}$ is a model of $\mathcal{S}$. To verify (M.1) given $i, j \in I$ with $i \prec j$ and $s \in \zeta(i, j)$, we have

$$
\varphi\left(\Phi^{\prime}(i, j, s)\right)= \begin{cases}\varphi\left(\Phi_{1}^{\prime}(i, j, s)\right)=s & \text { if } j \leq c \\ \varphi(\Phi(i, j, s))=s & \text { if } j=r\end{cases}
$$

which completes the verification of (M.1), For (M.2) consider $i, j \in I$ with $i \prec j$ and suppose that $j \leq c$. Then

$$
\begin{aligned}
w^{\prime}(i, j) & =w^{\prime}\left[\iota^{\prime}(i), \iota^{\prime}(j)\left[=w^{\prime}\left[\iota_{1}^{\prime}(i), \iota_{1}^{\prime}(j)[\right.\right.\right. \\
& =w_{1}^{\prime}\left[\iota_{1}^{\prime}(i), \iota_{1}^{\prime}(j)\left[=\left(w_{1}^{\prime} z^{\prime}\right)\left[\iota_{1}^{\prime}(i), \iota_{1}^{\prime}(j)[\right.\right.\right. \\
& =\left(w_{1}^{\prime} z^{\prime}\right)(i, j) .
\end{aligned}
$$

Therefore $\vec{c}\left(w^{\prime}(i, j)\right)=\chi(i, j)$ by (M.2) applied to the model $\mathcal{M}_{1}^{\prime}$ of $\mathcal{S}_{1}$. Suppose now that $j=r$, whence $i=c$. In this case $w^{\prime}(i, j)=w^{\prime}\left[\iota_{1}^{\prime}(c), \alpha_{w^{\prime}}+1[=\right.$ $w_{2}^{\prime}$. If $\zeta(c, r)=\emptyset$, then $\vec{c}\left(w^{\prime}(i, j)\right)=\vec{c}\left(w_{2}^{\prime}\right)=\chi(c, r)$ by the choice of $w_{2}^{\prime}$. Otherwise $w_{2}^{\prime}=\Phi^{\prime}\left(c, r, s_{0}\right)$ and so $\vec{c}\left(w^{\prime}(i, j)\right)=\vec{c}\left(\Phi^{\prime}\left(c, r, s_{0}\right)\right)=\vec{c}\left(\Phi\left(c, r, s_{0}\right)\right)=$ $\chi(c, r)$ again by (M.2) since $\mathcal{M}$ is a model of $\mathcal{S}$. This completes the verification of (M.2) Conditions (M.3) and (M.4) may be verified similarly to (M.2). Hence the induction step is also achieved in this case.

Case 4. This case handles the easiest situation where there is an elastic rightmost boundary equation (and Cases 10 13 do not apply): suppose that there is some elastic boundary equation $(i, \mathrm{x}, j, \overline{\mathrm{x}}) \in \mathcal{B}$ such that $\operatorname{right}(\mathrm{x})=$ $\operatorname{right}(\overline{\mathrm{x}})=\max I, i<j$, and

$$
c(w(i, j)) \varsubsetneqq c(w(i, \operatorname{right}(\mathbf{x}))) .
$$

In this case, it will be possible to obtain $\mathcal{S}_{1}$ by shrinking this boundary equation. Let $r=\max I$. Notice that, by (M.4) R satisfies $w(i, r)=$ $w(j, r)$. There are factorizations $w(i, r)=u_{i} v$ and $w(j, r)=u_{j} v$ such that $c\left(u_{i}\right)=c\left(u_{j}\right)=c(w(i, j))$ and the first letter of $v$ does not belong to $c\left(u_{i}\right)$. The situation is shown in Figure 4 where the new position $k$, which is to be defined below along with y and $\bar{y}$, will correspond, through the appropriate order preserving injective function $\iota_{1}$, to the end of $u_{i}$ and $u_{j}$. In this figure and the following ones, dashed lines indicate relevant positions, or outline boxes which have been cut out.

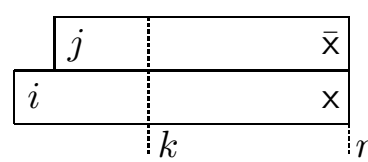

(a)

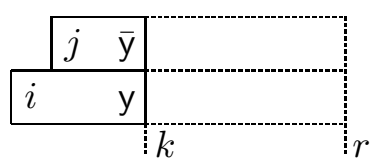

(b)

Figure 4: Case $4(a)$ in $\mathcal{S}$, and $(b)$ in $\mathcal{S}_{1}$. 
By Corollary 4.13 R satisfies $u_{i}=u_{j}$. We construct the pair $\left(\mathcal{S}_{1}, \mathcal{M}_{1}\right)$ as follows.

- We introduce a new variable $\mathrm{y}$ along with its dual $\overline{\mathrm{y}}$ and we let $\mathrm{X}_{1}=$ $\mathrm{X} \uplus\{\mathrm{y}, \overline{\mathrm{y}}\}$.

- Let $z=w\left[0, \iota(i)\left[\right.\right.$ and let $\beta$ be the ordinal such that $w\left[0, \beta\left[=z u_{i}\right.\right.$, that is, $\beta=\alpha_{z u_{i}}$.

- Consider the two factorization schemes $(I, \iota, \Phi)$ and $\left(\{\beta\},\{\beta\} \hookrightarrow \alpha_{w}+\right.$ $1, \emptyset)$ for $w$. By Proposition 8.1 there exists a common refinement $\left(I_{1}, \iota_{1}, \Phi_{1}\right)$. Let $k=\imath_{1}^{-1}(\beta)$.

- For $i, j \in I_{1}$ such that $i \prec j$, we set $\chi_{1}(i, j)=\vec{c}\left(w\left[\iota_{1}(i), \iota_{1}(j)[)\right.\right.$.

- We define the function $\operatorname{right}_{1}: \mathrm{X}_{1} \rightarrow I_{1}$ by letting $\operatorname{right}_{1}(\mathrm{y})=\operatorname{right}_{1}(\overline{\mathrm{y}})=$ $k$ and $\operatorname{right}_{1}(\mathbf{z})=\operatorname{right}(\mathbf{z})$ for $\mathbf{z} \in \mathbf{X}$ (here, in order to simplify the notation, we follow the convention of identifying $I$ with a subset of $I_{1}$ ).

- For $i, j \in I_{1}$ with $i \prec j$, we let

$$
\zeta_{1}(i, j)=\left\{s \in S: \Phi_{1}(i, j, s) \text { is defined }\right\} .
$$

- Finally, we obtain $\mathcal{B}_{1}$ from $\mathcal{B}$ by replacing the boundary equation $(i, \mathrm{x}, j, \overline{\mathrm{x}})$ and its dual by the new boundary equation $(i, \mathrm{y}, j, \overline{\mathrm{y}})$ along with its dual.

Proposition 9.1 The triple $\mathcal{M}_{1}=\left(w, \iota_{1}, \Phi_{1}\right)$ is a model of the system of boundary equations $\mathcal{S}_{1}=\left(\mathrm{X}_{1}, I_{1}, \zeta_{1}, \chi_{1}\right.$, right $\left.{ }_{1}, \mathcal{B}_{1}\right)$ such that the properties (P.1) and (P.2) hold.

Proof. We first check that $\mathcal{M}_{1}$ is a model of $\mathcal{S}_{1}$. Properties (M.1) and (M.3) of the definition of model follow from the fact that $\left(I_{1}, \iota_{1}, \Phi_{1}\right)$ is a factorization scheme for $w$. Property (M.2) is guaranteed by the definition of $\chi_{1}$ and Property (M.4) follows from the fact that this property holds for the model $\mathcal{M}$ of $\mathcal{S}$ since $\mathrm{R} \models u_{i}=u_{j}$.

Property (P.1) holds since we did not change the pseudoword in the model but we reduced by 2 the number of boxes $(i, \mathrm{x})$ whose right is the maximum index.

For Property (P.2) suppose that $\mathcal{M}_{1}^{\prime}=\left(w^{\prime}, \iota_{1}^{\prime}, \Phi_{1}^{\prime}\right)$ is a model of $\mathcal{S}_{1}$ in $\kappa$ terms. Let $\left(I, \iota^{\prime}, \Phi^{\prime}\right)$ be a restriction of the factorization scheme $\left(I_{1}, \iota_{1}^{\prime}, \Phi_{1}^{\prime}\right)$, where $\iota^{\prime}: I \rightarrow \alpha_{w^{\prime}}+1$ is the composite function $\iota_{1}^{\prime} \circ \iota_{1}^{-1} \circ \iota$. It remains to check that Properties (M.1) (M.4) of the definition of model are verified for $\mathcal{M}^{\prime}=\left(w^{\prime}, \iota^{\prime}, \Phi^{\prime}\right)$. Again Properties (M.1) and (M.3) follow from the fact that $\left(I, \iota^{\prime}, \Phi^{\prime}\right)$ is a factorization scheme for $w^{\prime}$.

For Property (M.2), suppose that $i, j \in I$ are such that $i \prec j$. Let $i_{1}, j_{1} \in I_{1}$ be such that $i_{1} \prec j_{1}$ and $\iota_{1}\left(i_{1}\right)=\iota(i)$ and $\iota_{1}\left(j_{1}\right)=\iota(j)$. Then $\iota_{1}^{\prime}\left(i_{1}\right)=\iota^{\prime}(i)$, 
$\iota_{1}^{\prime}\left(j_{1}\right)=\iota^{\prime}(j)$ and we obtain the following equalities:

$$
\begin{aligned}
\vec{c}\left(w ^ { \prime } \left[\iota^{\prime}(i), \iota^{\prime}(j)[)\right.\right. & =\vec{c}\left(w ^ { \prime } \left[\iota_{1}^{\prime}\left(i_{1}\right), \iota_{1}^{\prime}\left(j_{1}\right)[)=\chi_{1}\left(i_{1}, j_{1}\right)\right.\right. \\
& =\vec{c}\left(w \left[\iota_{1}\left(i_{1}\right), \iota_{1}\left(j_{1}\right)[)=\vec{c}(w[\iota(i), \iota(j)[)\right.\right. \\
& =\chi(i, j) .
\end{aligned}
$$

Finally, Property (M.4) is immediate for all but the boundary equation $(i, \mathrm{x}, j, \overline{\mathrm{x}})$ and its dual which amounts to $\mathrm{R} \models w^{\prime}(i, \operatorname{right}(\mathrm{x}))=w^{\prime}(j, \operatorname{right}(\overline{\mathrm{x}}))$. This follows from the condition $\mathrm{R} \models w^{\prime}(i, \operatorname{right}(\mathrm{y}))=w^{\prime}(j, \operatorname{right}(\overline{\mathrm{y}}))$ which is associated with the boundary equation $(i, \mathrm{y}, j, \overline{\mathrm{y}})$ by multiplying both sides on the right by $w^{\prime}\left[\iota_{1}^{\prime}(k), \iota^{\prime}(r)[\right.$.

Proposition 9.1 achieves the induction step in Case 4.

At this point, we introduce the announced auxiliary step. It will be used to transform the system and model in both Cases 5 and 6 .

\section{Auxiliary step}

We assume that Cases 14 do not apply, that there is at least one elastic boundary equation $(i, \mathrm{x}, j, \overline{\mathrm{x}})$ such that $i<j, \operatorname{right}(\mathrm{x})=\operatorname{right}(\overline{\mathrm{x}})=\max I$, and, for every such boundary equation, $c(w(i, j))=c(w(i, r))$, where $r=$ $\max I$. Hence, $\mathrm{R} \models w(j, r)=w(i, r)=w(i, j) w(j, r)$, so that $c(w(i, j))=$ $c(w(i, r))=\vec{c}(w(i, r))$. Among the variables $\mathrm{x} \operatorname{such}$ that $\operatorname{right}(\mathrm{x})=\operatorname{right}(\overline{\mathrm{x}})=$ $r$, we consider those for which left $(\mathrm{x})$ is minimum, and we denote this minimum by $\ell$. Let

$$
k_{0}=\min \{j \in I: \exists(\ell, \mathrm{x}, j, \overline{\mathrm{x}}) \in \mathcal{B} \text { and } \operatorname{right}(\mathrm{x})=\operatorname{right}(\overline{\mathrm{x}})=r\} .
$$

Note that $\ell$ and $k_{0}$ are well defined by the definition of left. We choose $\mathrm{x}_{0} \in \mathrm{X}$ such that $\left(\ell, \mathrm{x}_{0}, k_{0}, \overline{\mathrm{x}}_{0}\right) \in \mathcal{B}$ and $\operatorname{right}\left(\mathrm{x}_{0}\right)=\operatorname{right}\left(\overline{\mathrm{x}}_{0}\right)=r$. Since Case 2 does not apply, we have $\ell<k_{0}$.

The auxiliary step takes as input the original system and the boundary equation $\left(\ell, \mathrm{x}_{0}, k_{0}, \overline{\mathrm{x}}_{0}\right)$, and outputs a new system, where $\left(\ell, \mathrm{x}_{0}, k_{0}, \overline{\mathrm{x}}_{0}\right)$ and its dual are removed and replaced by two new boundary equations and their duals.

Let $i_{0} \in I \backslash\{r\}$. If $i_{0}<\ell$, then we set $n\left(\mathcal{S}, \mathcal{M}, i_{0}\right)=-\infty$. Suppose now that $i_{0} \geq \ell$. Since $c\left(w\left(\ell, k_{0}\right)\right)=c(w(\ell, r))$, we have $\mathrm{R} \models w(\ell, r)=$ $w\left(\ell, k_{0}\right) w\left(k_{0}, r\right)=w\left(k_{0}, r\right)=w\left(\ell, k_{0}\right)^{\omega}$. Therefore, by Theorem4.10, $\alpha_{w(\ell, r)}=$ $\alpha_{w\left(\ell, k_{0}\right)^{\omega}}$. Since $i_{0} \neq r$, we have $\alpha_{w\left(\ell, i_{0}\right)}<\alpha_{w(\ell, r)}=\alpha_{w\left(\ell, k_{0}\right)^{\omega}}=\alpha_{w\left(\ell, k_{0}\right)} \cdot \omega$, so there exists an integer $n$ such that $\alpha_{w\left(\ell, k_{0}\right)} \cdot n<\alpha_{w\left(\ell, i_{0}\right)}<\alpha_{w\left(\ell, k_{0}\right)} \cdot(n+1)$. A repeated application of Fact 5.3 then yields factorizations $w\left(\ell, i_{0}\right)=$ $u_{1} \cdots u_{n} v_{1}$ and $w\left(i_{0}, r\right)=v_{2} v_{3}$ such that the first letter of each factor in 
$u_{1} \cdots u_{n} v_{1} v_{2} v_{3}$ does not belong to the cumulative content of the preceding factor and

$$
\mathrm{R} \models u_{1}=\cdots=u_{n}=v_{1} v_{2}=w\left(\ell, k_{0}\right) .
$$

We set $n\left(\mathcal{S}, \mathcal{M}, i_{0}\right)=n$, where $n$ is determined by (9.1).

In the case where $i_{0} \geq \ell$, we will define a pair $\left(\mathcal{S}_{(1)}, \mathcal{M}_{(1)}\right)$, consisting of a system of boundary equations $\mathcal{S}_{(1)}$, whose index set $I_{(1)}$ contains $I$, and a model $\mathcal{M}_{(1)}$ of $\mathcal{S}_{(1)}$, such that $\left[\mathcal{S}_{(1)}, \mathcal{M}_{(1)}\right]=[\mathcal{S}, \mathcal{M}]$, none of the Cases $1-$ 4 apply to $\left(\mathcal{S}_{(1)}, \mathcal{M}_{(1)}\right)$, whenever $\mathcal{S}_{(1)}$ has a model in $\kappa$-terms so does $\mathcal{S}$, and $n\left(\mathcal{S}_{(1)}, \mathcal{M}_{(1)}, i_{0}\right)<n\left(\mathcal{S}, \mathcal{M}, i_{0}\right)$. Once we have managed to obtain such a construction, we may conclude, inductively, that it suffices to consider the case in which $i_{0}<\ell$.

We construct the pair $\left(\mathcal{S}_{(1)}, \mathcal{M}_{(1)}\right)$ as follows. We let

$$
\begin{aligned}
\mathcal{S}_{(1)} & =\left(\mathrm{X}_{(1)}, I_{(1)}, \zeta_{(1)}, \chi_{(1)}, \operatorname{right}_{(1)}, \mathcal{B}_{(1)}\right) \\
\mathcal{M}_{(1)} & =\left(w, \iota_{(1)}, \Phi_{(1)}\right)
\end{aligned}
$$

be defined as follows. The construction resembles considerably the one adopted for Case 4

- We consider four new variables $\mathrm{y}_{1}, \overline{\mathrm{y}}_{1}, \mathrm{y}_{2}, \overline{\mathrm{y}}_{2}$ and we take $\mathrm{X}_{(1)}=\mathrm{X} \uplus$ $\left\{\mathrm{y}_{1}, \overline{\mathrm{y}}_{1}, \mathrm{y}_{2}, \overline{\mathrm{y}}_{2}\right\}$.

- Let $\beta=\iota\left(k_{0}\right)+\left(\iota\left(k_{0}\right)-\iota(\ell)\right)$.

- Consider the two factorization schemes $(I, \iota, \Phi)$ and $\left(\{\beta\},\{\beta\} \hookrightarrow \alpha_{w}+\right.$ $1, \emptyset)$ for $w$. By Proposition 8.1 there exists a common refinement $\left(I_{(1)}, \iota_{(1)}, \Phi_{(1)}\right)$. Let $k_{1}=i_{(1)}^{-1}(\beta)$.

- For $i, j \in I_{(1)}$ such that $i \prec j$, we set $\chi_{(1)}(i, j)=\vec{c}\left(w\left[\iota_{(1)}(i), \iota_{(1)}(j)[)\right.\right.$.

- We define the function $\operatorname{right}_{(1)}: \mathrm{X}_{(1)} \rightarrow I_{(1)}$ by letting $\operatorname{right}_{(1)}\left(\mathrm{y}_{1}\right)=k_{0}$, $\operatorname{right}_{(1)}\left(\overline{\mathrm{y}}_{1}\right)=k_{1}, \operatorname{right}_{(1)}\left(\mathrm{y}_{2}\right)=\operatorname{right}_{(1)}\left(\overline{\mathrm{y}}_{2}\right)=r, \operatorname{and}_{\operatorname{right}}^{(1)}(\mathrm{x})=\operatorname{right}(\mathrm{x})$ for $x \in X$.

- For $i, j \in I_{(1)}$ with $i \prec j$, we let

$$
\zeta_{(1)}(i, j)=\left\{s \in S: \Phi_{(1)}(i, j, s) \text { is defined }\right\} .
$$

- Finally, we obtain $\mathcal{B}_{(1)}$ from $\mathcal{B}$ by replacing $\left(\ell, \mathrm{x}_{0}, k_{0}, \bar{x}_{0}\right)$ and its dual by two new boundary equations $\left(\ell, \mathrm{y}_{1}, k_{0}, \overline{\mathrm{y}}_{1}\right)$ and $\left(k_{0}, \mathrm{y}_{2}, k_{1}, \overline{\mathrm{y}}_{2}\right)$, along with their duals. We will refer to this procedure as pushing forward the $\operatorname{period}\left(\ell, k_{0}\right)$.

The boundary equations that get transformed are shown in Figure 5 It is now routine to check the following result using Lemma 6.1 


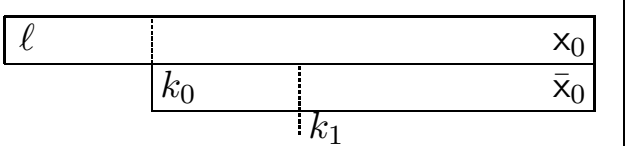

(a)

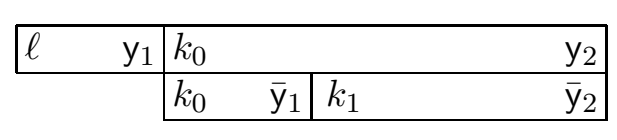

(b)

Figure 5: Auxiliary step: $(a)$ in $\mathcal{S}$ and $(b)$ in $\mathcal{S}_{(1)}$, while pushing forward one period.

Proposition 9.2 The triple $\mathcal{M}_{(1)}=\left(w, \iota_{(1)}, \Phi_{(1)}\right)$ is a model of the system of boundary equations $\mathcal{S}_{(1)}=\left(\mathrm{X}_{(1)}, I_{(1)}, \zeta_{(1)}, \chi_{(1)}\right.$, right $\left.(1), \mathcal{B}_{(1)}\right)$ such that the following properties are verified:

(1) if $\mathcal{S}_{(1)}$ admits a model in $\kappa$-terms, then so does $\mathcal{S}$;

(2) $n\left(\mathcal{S}_{(1)}, \mathcal{M}_{(1)}, i_{0}\right)<n\left(\mathcal{S}, \mathcal{M}, i_{0}\right)$;

(3) $\left[\mathcal{S}_{(1)}, \mathcal{M}_{(1)}\right]=[\mathcal{S}, \mathcal{M}]$.

Case 5. We now consider the case where all elastic boundary equations whose right value is $\max I$ do not fall in Case 4, and there is a nonelastic boundary equation, one of whose boxes has a maximal right value. In this case, the outline of the procedure to reduce the parameter $[\mathcal{S}, \mathcal{N}]$ consists in choosing a suitable such boundary equation, and in matching its associated boxes, by "transporting" some factorization points appearing inside the rightmost of these boxes to the leftmost one. This is justified by the results of Section 4 since both boxes represent factors of the solution which are equal over R. This matching can be seen, graphically, as moving the rightmost of the two boxes to align it with the other one. After this, we will drop the rightmost box, thus decreasing the induction parameter.

Let $r=\max I$. Suppose that there exists at least one variable $\mathrm{x}$ such that $\operatorname{right}(\mathrm{x})=r \neq \operatorname{right}(\overline{\mathrm{x}})$. Let

$$
\ell=\min \{\operatorname{left}(\mathrm{x}): \mathrm{x} \in \mathrm{X}, \operatorname{right}(\mathrm{x})=r \neq \operatorname{right}(\overline{\mathrm{x}})\} .
$$

Since we are assuming that Cases 10 10 not apply, note that $\ell$ is well defined and $\ell<r$. Moreover, by applying repeatedly Proposition 9.2 of the auxiliary step to push forward periods, we may assume that all elastic boundary equations $(i, \mathrm{x}, j, \overline{\mathrm{x}})$ with $\operatorname{right}(\mathrm{x})=\operatorname{right}(\overline{\mathrm{x}})=r$ are such that $\ell<i$ (hence also $\ell<j$, by duality).

Choose $\mathrm{x}_{0} \in \mathrm{X}$ such that left $\left(\mathrm{x}_{0}\right)=\ell$ and $\operatorname{right}\left(\mathrm{x}_{0}\right)=r \neq \operatorname{right}\left(\overline{\mathrm{x}}_{0}\right)$. Let $\ell^{*}$ be any element of $I$ such that $\left(\ell, \mathrm{x}_{0}, \ell^{*}, \overline{\mathrm{x}}_{0}\right) \in \mathcal{B}$ and let $r^{*}=\operatorname{right}\left(\bar{x}_{0}\right)$. The critical boundary is the element $c=\max \left\{c^{\prime}, \ell\right\}$ of $I$, where

$$
c^{\prime}=\max \{\operatorname{right}(\mathrm{x}): \mathrm{x} \in \mathrm{X}, \operatorname{left}(\mathrm{x})<\ell\} .
$$

Since $\operatorname{right}\left(\mathrm{x}_{0}\right)=\max I \neq \operatorname{right}\left(\overline{\mathrm{x}}_{0}\right)$, we have $\operatorname{right}\left(\mathrm{x}_{0}\right)>\operatorname{right}\left(\overline{\mathrm{x}}_{0}\right)$. By $(\mathrm{S} .2)$, it follows that $\ell=\operatorname{left}\left(\mathrm{x}_{0}\right)>\ell^{*}$. Hence $r^{*}=\operatorname{right}\left(\bar{x}_{0}\right)$ belongs to the set of 
which $c^{\prime}$ is defined to be the maximum, so that $c^{\prime}$ and $c$ are both well defined and $r^{*} \leq c^{\prime} \leq c$. We also have $\ell^{*}<\ell \leq c<r$.

A representation is given in Figure [6 $(a)$. The box $(i, \mathrm{y})$ of the second row is such that right(y) is maximum among all right values of boxes starting before $\ell$, hence $c=\operatorname{right}(\mathrm{y})$.

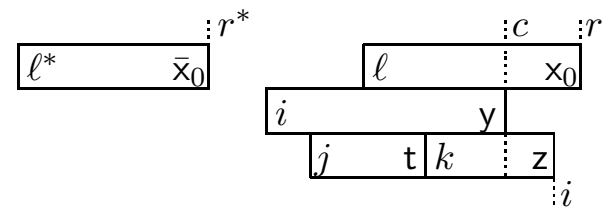

(a)

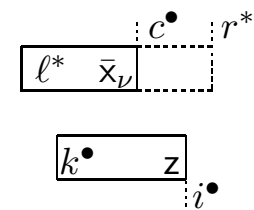

(b)

Figure 6: Case 5] (a) before and (b) after transporting the box $\left(\ell, \mathrm{x}_{0}\right)$.

The set of transport positions is the subset $T$ of $I$ defined by

$$
T=\{i \in I: c \leq i\} \cup\{i \in I: \exists \operatorname{a} \text { box }(i, \mathrm{x}) \text { such that } \operatorname{right}(\mathrm{x})>c\} .
$$

In the example of Figure 6 (a), the set $T$ is $\{c, i, r\} \cup\{k, \ell\}$, since the boxes "crossing" the critical boundary $c$ are $(k, \mathrm{z})$ and $\left(\ell, \mathrm{x}_{0}\right)$. We note that:

(i) $\max T=r$, since $r=\max I$ and $r \in T$;

(ii) for every box $\left(i, \mathrm{x}_{0}\right), i \in T$;

(iii) by definition of $c, \operatorname{right}(\mathrm{x})>c \operatorname{implies} \operatorname{left}(\mathrm{x}) \geq \ell$, and so $\ell=\min T$.

For $i \in T$, let $i^{\circ}=\iota\left(\ell^{*}\right)+(\iota(i)-\iota(\ell))$. Note that $\ell^{\circ}=\iota\left(\ell^{*}\right)$. Likewise, since $\mathcal{M}$ is a model of $\mathcal{S}, \mathrm{R}$ satisfies the pseudoidentity $w\left(\ell^{*}, r^{*}\right)=w(\ell, r)$ by (M.4) so $r^{\circ}=\iota\left(r^{*}\right)<\iota(r)$.

Lemma 9.3 The function $i \mapsto i^{\circ}$ enjoys the following properties.

(1) The function is order-preserving and injective.

(2) If $\ell \leq i \leq r$ then $\mathrm{R}$ satisfies the pseudoidentity $w\left[\ell^{\circ}, i^{\circ}[=w(\ell, i)\right.$.

(3) For every $i \in T$, we have $i^{\circ}<\iota(i)$.

Proof. Taking into account that $\iota$ is order-preserving and injective, property (1) is an exercise in ordinal arithmetic. Note that the hypothesis that $\mathrm{R}$ satisfies the pseudoidentity $w\left(\ell^{*}, r^{*}\right)=w(\ell, r)$ implies

$$
r^{\circ}-\ell^{\circ}=\iota(r)-\iota(\ell)
$$

and also $w\left[\ell^{\circ}, i^{\circ}[=w(\ell, i)\right.$ using Corollary 4.13 and the definition of the function $i \mapsto i^{\circ}$, which establishes (2). Finally, consider the following ordinal inequalities and equalities: $\ell^{\circ} \leq i^{\circ} \leq r^{\circ}, \iota(\ell) \leq \iota(i) \leq \iota(r), r^{\circ}<\iota(r)$, and

$$
i^{\circ}-\ell^{\circ}=\iota(i)-\iota(\ell) \text {. }
$$


Since equal summands can be canceled on the left in ordinal sums, it follows from (9.2) and (9.3) that $r^{\circ}-i^{\circ}=\iota(r)-\iota(i)$. As $r^{\circ}<\iota(r)$, Lemma 4.11 yields that $i^{\circ}<\iota(i)$, which proves $(3)$

Consider two factorization schemes for $w$,

$$
\left(I_{(1)}, \iota_{(1)}, \Phi_{(1)}\right) \text { and }\left(I_{(2)}, \iota_{(2)}, \Phi_{(2)}\right),
$$

where $\left(I_{(1)}, \iota_{(1)}, \Phi_{(1)}\right)$ is obtained from $(I, \iota, \Phi)$ by restriction (cf. Proposition 8.2) and

- $I_{(1)}=\{i \in I: i \leq c\}$ and $I_{(2)}=\left\{i^{\circ}: i \in T\right\}$;

- $\iota_{(1)}=\iota_{I_{(1)}}$ and $\iota_{(2)}: I_{(2)} \hookrightarrow \alpha_{w}+1$ is the inclusion mapping;

- let $\left(T,\left.\iota\right|_{T}, \Phi_{2}\right)$ be obtained from the factorization scheme $(I, \iota, \Phi)$ by restriction; for $i, j \in T$ such that $i \prec j$, we let $\Phi_{(2)}\left(i^{\circ}, j^{\circ}, s\right)=\Phi_{2}(i, j, s)$ whenever the latter is defined.

By Lemma 9.3] $(2)\left(I_{(2)}, \iota_{(2)}, \Phi_{(2)}\right)$ is indeed a factorization scheme. Informally, $I_{(2)}$ represents transport positions shifted from $[\ell, r]$ to $\left[\ell^{*}, r^{*}\right]$. By Proposition 8.1 there exists a common refinement $\left(I_{1}, \iota_{1}, \Phi_{1}\right)$ of the two factorization schemes (9.4).

We proceed to define the new system of boundary equations

$$
\mathcal{S}_{1}=\left(\mathrm{X}_{1}, I_{1}, \zeta_{1}, \chi_{1}, \text { right }_{1}, \mathcal{B}_{1}\right) .
$$

To avoid ambiguity in the notation $w(i, j)$, we may assume, without loss of generality, that $I$ is disjoint from $I_{1}$. Denote the composite $\iota_{1}^{-1} \circ \iota$ by $\xi$. To simplify the notation, we also let $i^{\bullet}=\iota_{1}^{-1}\left(i^{\circ}\right)$ whenever $i \in T$.

- The set $\mathrm{X}_{1}$ is obtained by adding a new variable $\mathrm{x}_{\nu}$ along with its dual $\overline{\mathrm{x}}_{\nu}$.

- For $i, j \in I_{1}$, we let

$$
\zeta_{1}(i, j)=\left\{s \in S: \Phi_{1}(i, j, s) \text { is defined }\right\} .
$$

- For $i, j \in I_{1}$ with $i \prec j$, we let $\chi_{1}(i, j)=\vec{c}(w(i, j))$.

- The function right $1: \mathrm{X}_{1} \rightarrow I_{1}$ is defined by

$$
\operatorname{right}_{1}(\mathrm{x})= \begin{cases}\xi(\operatorname{right}(\mathrm{x})) & \text { if } \mathrm{x} \in \mathrm{X} \text { and } \operatorname{right}(\mathrm{x}) \leq c \\ (\operatorname{right}(\mathrm{x}))^{\bullet} & \text { if } \mathrm{x} \in \mathrm{X} \text { and } \operatorname{right}(\mathrm{x})>c \\ \xi(c) & \text { if } \mathrm{x}=\mathrm{x}_{\nu} \\ c^{\bullet} & \text { if } \mathrm{x}=\overline{\mathrm{x}}_{\nu} .\end{cases}
$$

The description of the set $\mathcal{B}_{1}$ of boundary equations is somewhat more complicated and proceeds in several stages, starting with $\mathcal{B}_{1}=\emptyset$. 
(a) let $\mathcal{B}^{\prime}=\mathcal{B} \backslash\left\{\left(\ell, \mathrm{x}_{0}, \ell^{*}, \overline{\mathrm{x}}_{0}\right),\left(\ell^{*}, \overline{\mathrm{x}}_{0}, \ell, \mathrm{x}_{0}\right)\right\}$;

(b) put a new boundary equation $\left(\xi(\ell), \mathrm{x}_{\nu}, \ell^{\bullet}, \overline{\mathrm{x}}_{\nu}\right)$ and its dual $\left(\ell^{\bullet}, \overline{\mathrm{x}}_{\nu}, \xi(\ell), \mathrm{x}_{\nu}\right)$ into $\mathcal{B}_{1}$;

(c) for each variable $\mathrm{x} \in \mathrm{X}$ such that $\operatorname{right}(\mathrm{x})>c$ and each boundary equation in $\mathcal{B}^{\prime}$ of the form $(i, \mathrm{x}, j, \overline{\mathrm{x}})$, put new boundary equations into $\mathcal{B}_{1}$ as follows:

(i) if $\operatorname{right}(\overline{\mathrm{x}}) \leq c$, then add to $\mathcal{B}_{1}$ the 4-tuple $\left(i^{\bullet}, \mathrm{x}, \xi(j), \overline{\mathrm{x}}\right)$ and its dual $\left(\xi(j), \bar{x}, i^{\bullet}, \mathrm{x}\right)$;

(ii) if $\operatorname{right}(\overline{\mathrm{x}})>c$, then add to $\mathcal{B}_{1}$ the 4 -tuple $\left(i^{\bullet}, \mathrm{x}, j^{\bullet}, \overline{\mathrm{x}}\right)$ and its dual $\left(j \bullet, \bar{x}, i^{\bullet}, \mathrm{x}\right)$;

(d) for each variable $\mathrm{x} \in \mathrm{X}$ such that both $\operatorname{right}(\mathrm{x}) \leq c$ and $\operatorname{right}(\overline{\mathrm{x}}) \leq c$, and each boundary equation in $\mathcal{B}^{\prime}$ of the form $(i, x, j, \bar{x})$, put a new boundary equation $(\xi(i), \mathrm{x}, \xi(j), \overline{\mathrm{x}})$ into $\mathcal{B}_{1}$.

Figure [6 (b) shows the system of boundary equations obtained from the one of Figure 6 6 (a). (To simplify notation, we wrote $j, \ell, c, i$, and $r$ for $\xi(j)$, $\xi(\ell), \xi(c), \xi(i)$, and $\xi(r)$, respectively.)

Finally, we let $\mathcal{M}_{1}=\left(w_{1}, \iota_{1}, \Phi_{1}\right)$, where $w_{1}=w[0, \iota(c)[$. Notice that $\max I_{1}=\xi(c)$ and that $\iota_{1}(\xi(c))=\iota(c)=\alpha_{w_{1}}$.

Proposition 9.4 The tuple $\mathcal{S}_{1}$ defined by (9.5) is a system of boundary equations and $\mathcal{M}_{1}$ is a model of $\mathcal{S}_{1}$.

Proof. To check that $\mathcal{M}_{1}$ is a model of $\mathcal{S}_{1}$, we need to verify conditions (M.1) (M.4) The first three are immediate from the definitions of $\zeta_{1}, \Phi_{1}$, and $\chi_{1}$. For condition (M.4), we consider the various types of boundary equations as given in steps $(b),(c)$, and $(d)$.

In case $(b)$, consider the boundary equation $\left(\xi(\ell), \mathrm{x}_{\nu}, \ell^{\bullet}, \overline{\mathrm{x}}_{\nu}\right)$. By definition of $\mathcal{M}_{1}$, we have $w_{1}\left(\xi(\ell)\right.$, $\left.\operatorname{right}_{1}\left(\mathrm{x}_{\nu}\right)\right)=w_{1}(\ell, c)$ and the pseudoidentity which we must prove to be valid in $\mathrm{R}$ becomes $w_{1}(\ell, c)=w_{1}\left[\ell^{\circ}, c^{\circ}\right.$, which follows from Lemma 9.3)

In case $(c)(i)$ we consider $(i, \mathrm{x}, j, \overline{\mathrm{x}}) \in \mathcal{B}^{\prime} \operatorname{such}$ that $\operatorname{right}(\overline{\mathrm{x}}) \leq c<\operatorname{right}(\mathrm{x})$. We need to prove that $\mathrm{R} \models w_{1}\left(i, \operatorname{right}_{1}(\mathbf{x})\right)=w_{1}\left(\xi(j), \operatorname{right}_{1}(\overline{\mathrm{x}})\right)$, which amounts to prove that $\mathrm{R} \models w\left(i, \operatorname{right}_{1}(\mathrm{x})\right)=w\left(\xi(j), \operatorname{right}_{1}(\overline{\mathrm{x}})\right)$. Now, $\operatorname{right}_{1}(\mathrm{x})=(\operatorname{right}(\mathrm{x}))^{\bullet}$ and $\operatorname{right}_{1}(\overline{\mathrm{x}})=\xi(\operatorname{right}(\overline{\mathrm{x}}))$ so that, by Lemma 9.3](2) and the definition of the model $\mathcal{M}_{1}$, the pseudoidentity whose validity in $\mathrm{R}$ we need to establish becomes $w(i, \operatorname{right}(\mathbf{x}))=w(j, \operatorname{right}(\overline{\mathrm{x}}))$, which is valid since $\mathcal{M}$ is a model of $\mathcal{S}$. Cases $(c)((i i)$ and $(d)$ are handled similarly.

Proposition 9.5 The properties (P.1) and (P.2) are verified by the step $(\mathcal{S}, \mathcal{M}) \mapsto\left(\mathcal{S}_{1}, \mathcal{M}_{1}\right)$. 
Proof. For property (P.1) it suffices to notice that $\alpha_{w_{1}}=\iota(c)<\iota(r)=\alpha_{w}$. Suppose that $\mathcal{M}_{1}^{\prime}=\left(w_{1}^{\prime}, \iota_{1}^{\prime}, \Phi_{1}^{\prime}\right)$ is a model of $\mathcal{S}_{1}$ in $\kappa$-terms. We construct a model $\mathcal{M}^{\prime}=\left(w^{\prime}, \iota^{\prime}, \Phi^{\prime}\right)$ of $\mathcal{S}$ in $\kappa$-terms as follows.

- Let $w^{\prime}=w_{1}^{\prime} \cdot w_{1}^{\prime}\left(c^{\bullet}, r^{\bullet}\right)$. By Lemma 7.1 $w^{\prime}$ is a $\kappa$-term.

- Let $\iota^{\prime}: I \rightarrow \alpha_{w^{\prime}}+1$ be defined by

$$
\iota^{\prime}(i)= \begin{cases}\iota_{1}^{\prime}(\xi(i)) & \text { if } i<c \\ \alpha_{w_{1}^{\prime}}+\left(\iota_{1}^{\prime}\left(i^{\bullet}\right)-\iota_{1}^{\prime}\left(c^{\bullet}\right)\right) & \text { if } i \geq c .\end{cases}
$$

Note that $\iota^{\prime}(c)=\alpha_{w_{1}^{\prime}}=\iota_{1}^{\prime}(\xi(c))$ since $\mathcal{M}_{1}^{\prime}$ is a model of $\mathcal{S}_{1}$ and max $I_{1}=$ $\xi(c)$. Moreover $\iota^{\prime}(r)=\alpha_{w^{\prime}}$.

- We consider the following two restrictions of the factorization scheme $\left(I_{1}, \iota_{1}^{\prime}, \Phi_{1}^{\prime}\right)$ for $w^{\prime}:\left(I_{(1)},\left.\iota^{\prime}\right|_{I_{(1)}}, \Phi_{(1)}^{\prime}\right)$ and $\left(I_{(2)},\left.\iota^{\prime}\right|_{I_{(2)}}, \Phi_{(2)}^{\prime}\right)$. We transfer $\left(I_{(2)},\left.\iota^{\prime}\right|_{I_{(2)}}, \Phi_{(2)}^{\prime}\right)$ to a new factorization scheme $\left(T,\left.\iota^{\prime}\right|_{T}, \Phi_{2}^{\prime}\right)$ for $w^{\prime}$ by letting $\Phi_{2}^{\prime}(i, j, s)=\Phi_{(2)}^{\prime}\left(i^{\circ}, j^{\circ}, s\right)$ whenever $i, j \in T$ and $s \in S$ are such that $i \prec j$ in $T$ and $\Phi_{(2)}^{\prime}\left(i^{\circ}, j^{\circ}, s\right)$ is defined. By Proposition 8.1 there is a common refinement $(\bar{I}, \bar{\iota}, \bar{\Phi})$ of the factorization schemes for $w^{\prime}$ given by $\left(I_{1}, \iota_{1}^{\prime}, \Phi_{1}^{\prime}\right)$ and $\left(T,\left.\iota^{\prime}\right|_{T}, \Phi_{2}^{\prime}\right)$. Finally, we set

$$
\Phi^{\prime}(i, j, s)=\bar{\Phi}\left(\bar{\iota}^{-1}\left(\iota^{\prime}(i)\right), \bar{\iota}^{-1}\left(\iota^{\prime}(j)\right), s\right),
$$

whenever $i, j \in I$ and $s \in S$ are such that $i \prec j$ and the right side of (9.6) is defined.

The following pseudoidentities are valid in R:

$$
\begin{aligned}
w^{\prime}(\ell, r) & =w^{\prime}(\ell, c) w^{\prime}(c, r) \\
& =w^{\prime}\left[\iota^{\prime}(\ell), \iota^{\prime}(c)\left[\cdot w ^ { \prime } \left[\iota^{\prime}(c), \iota^{\prime}(r)[\right.\right.\right. \\
& =w^{\prime}\left[\iota_{1}^{\prime}(\xi(\ell)), \iota_{1}^{\prime}(\xi(c))\left[\cdot w ^ { \prime } \left[\alpha_{w_{1}^{\prime}}, \alpha_{w^{\prime}}[\right.\right.\right. \\
& =w_{1}^{\prime}(\xi(\ell), \xi(c)) w_{1}^{\prime}\left(c^{\bullet}, r^{\bullet}\right) \\
& =w_{1}^{\prime}\left(\ell^{\bullet}, c^{\bullet}\right) w_{1}^{\prime}\left(c^{\bullet}, r^{\bullet}\right) \text { in view of (M.4)] for }\left(\xi(\ell), \mathrm{x}_{\nu}, \ell^{\bullet}, \overline{\mathrm{x}}_{\nu}\right) \in \mathcal{B}_{1} \\
& =w_{1}^{\prime}\left(\ell^{\bullet}, r^{\bullet}\right) \\
& =w_{1}^{\prime}\left[\iota_{1}^{\prime}\left(\ell^{\bullet}\right), \iota_{1}^{\prime}\left(r^{\bullet}\right)[\right. \\
& =w^{\prime}\left[\iota^{\prime}\left(\ell^{*}\right), \iota^{\prime}\left(r^{*}\right)[\right. \\
& =w^{\prime}\left(\ell^{*}, r^{*}\right) .
\end{aligned}
$$

This shows that

$$
\mathrm{R} \models w^{\prime}(\ell, r)=w^{\prime}\left(\ell^{*}, r^{*}\right) .
$$

We proceed to verify that the properties (M.1) (M.4) hold for $\mathcal{M}^{\prime}$. Properties (M.1) and (M.3) follow from the fact that $\left(I, \iota^{\prime}, \Phi^{\prime}\right)$ is a factorization scheme for $w^{\prime}$, which is immediate to check taking into account (9.7). 
Let $i, j \in I$ with $i \prec j$. To verify (M.2) suppose first that $j \leq c$ and let $k \in I_{1}$ be such that $\xi(i) \leq k \prec \xi(j)$. Then we have

$$
\begin{aligned}
\vec{c}\left(w^{\prime}(i, j)\right) & =\vec{c}\left(w_{1}^{\prime}(k, \xi(j))\right)=\chi_{1}(k, \xi(j))=\vec{c}(w(k, \xi(j))) \\
& =\vec{c}(w(\xi(i), \xi(j)))=\vec{c}(w(i, j)) \\
& =\chi(i, j) .
\end{aligned}
$$

On the other hand, if $i \geq c$, then we let $k \in I_{1}$ be such that $i^{\bullet} \leq k \prec j^{\bullet}$. Now, we obtain the following equalities:

$$
\begin{aligned}
\vec{c}\left(w^{\prime}(i, j)\right) & =\vec{c}\left(w_{1}^{\prime}\left(i^{\bullet}, j^{\bullet}\right)\right) \\
& =\vec{c}\left(w_{1}^{\prime}\left(k, j^{\bullet}\right)\right)=\chi_{1}\left(k, j^{\bullet}\right)=\vec{c}\left(w\left(k, j^{\bullet}\right)\right) \\
& =\vec{c}\left(w\left(i^{\bullet}, j^{\bullet}\right)\right)=\vec{c}(w(i, j)) \quad \text { since R } \models w\left(\ell^{*}, r^{*}\right)=w(\ell, r) \\
& =\chi(i, j) .
\end{aligned}
$$

This establishes (M.2)

It remains to verify property (M.4) For the boundary equation $\left(\ell, \mathrm{x}_{0}, \ell^{*}, \overline{\mathrm{x}}_{0}\right)$, this is given by (9.7). Consider next a boundary equation $(i, \mathrm{x}, j, \overline{\mathrm{x}}) \in \mathcal{B}$ with $\ell \leq i$. In view of (M.4) for $\left(\xi(\ell), \mathrm{x}_{\nu}, \ell^{\bullet}, \overline{\mathrm{x}}_{\nu}\right) \in \mathcal{B}_{1}$, we know that $\mathrm{R}$ satisfies $w_{1}^{\prime}(\xi(\ell), \xi(c))=w_{1}^{\prime}\left(\ell^{\bullet}, c^{\bullet}\right)$. Using (9.3), we also have $\alpha_{w_{1}^{\prime}(\xi(\ell), \xi(i))}=$ $\alpha_{w_{1}^{\prime}(\ell, i \bullet)}$. Corollary 4.13 therefore implies that

$$
\mathrm{R} \models w_{1}^{\prime}(\xi(i), \xi(c))=w_{1}^{\prime}\left(i^{\bullet}, c^{\bullet}\right) .
$$

If $i \leq c<\operatorname{right}(\mathrm{x})$, then $\mathrm{R}$ satisfies the following pseudoidentities:

$$
\begin{aligned}
w^{\prime}(i, \operatorname{right}(\mathbf{x})) & =w^{\prime}(i, c) w^{\prime}(c, \operatorname{right}(\mathbf{x})) \\
& =w_{1}^{\prime}(\xi(i), \xi(c)) w_{1}^{\prime}\left(c^{\bullet},(\operatorname{right}(\mathbf{x}))^{\bullet}\right) \\
& =w_{1}^{\prime}\left(i^{\bullet}, c^{\bullet}\right) w_{1}^{\prime}\left(c^{\bullet},(\operatorname{right}(\mathbf{x}))^{\bullet}\right) \text { by (9.8) } \\
& =w_{1}^{\prime}\left(i^{\bullet},(\operatorname{right}(\mathbf{x}))^{\bullet}\right) .
\end{aligned}
$$

That $\mathrm{R}$ also satisfies $w^{\prime}(i, \operatorname{right}(\mathrm{x}))=w_{1}^{\prime}\left(i \bullet,(\operatorname{right}(\mathrm{x}))^{\bullet}\right)$ when $c<i$, is an immediate consequence of the definition of $\iota^{\prime}$. Hence

$$
\mathrm{R} \models w^{\prime}(i, \operatorname{right}(\mathbf{x}))=w_{1}^{\prime}\left(i^{\bullet},(\operatorname{right}(\mathbf{x}))^{\bullet}\right)
$$

Assume first that $\operatorname{right}(\overline{\mathrm{x}}) \leq c$. Then we have $(i \bullet, \mathrm{x}, \xi(j), \overline{\mathrm{x}}) \in \mathcal{B}_{1}$ and so $\mathrm{R}$ satisfies

$$
\begin{aligned}
w_{1}^{\prime}\left(i^{\bullet},(\operatorname{right}(\mathrm{x}))^{\bullet}\right) & =w_{1}^{\prime}\left(\xi(j), \operatorname{right}_{1}(\overline{\mathrm{x}})\right)=w_{1}^{\prime}(\xi(j), \xi(\operatorname{right}(\overline{\mathrm{x}}))) \\
& =w^{\prime}(j, \operatorname{right}(\overline{\mathrm{x}}))
\end{aligned}
$$

On the other hand, if $\operatorname{right}(\overline{\mathrm{x}})>c$ then $\left(i^{\bullet}, x, j^{\bullet}, \overline{\mathrm{x}}\right) \in \mathcal{B}_{1}$ and so $\mathrm{R}$ satisfies

$$
\begin{aligned}
w_{1}^{\prime}\left(i^{\bullet},(\operatorname{right}(\mathrm{x}))^{\bullet}\right) & =w_{1}^{\prime}\left(j^{\bullet}, \operatorname{right}_{1}(\overline{\mathrm{x}})\right)=w_{1}^{\prime}\left(j^{\bullet},(\operatorname{right}(\overline{\mathrm{x}}))^{\bullet}\right) \\
& =w^{\prime}(j, \operatorname{right}(\overline{\mathrm{x}})) .
\end{aligned}
$$


It remains to consider the case in which both $\operatorname{right}(\mathrm{x})$ and $\operatorname{right}(\overline{\mathrm{x}})$ are at most $c$. Then $(\xi(i), x, \xi(j), \bar{x}) \in \mathcal{B}_{1}$, $\operatorname{right}_{1}(\mathrm{x})=\xi(\operatorname{right}(\mathrm{x}))$, and $\operatorname{right}_{1}(\overline{\mathrm{x}})=$ $\xi(\operatorname{right}(\bar{x}))$. Hence R satisfies the following pseudoidentities:

$$
\begin{aligned}
w^{\prime}(i, \operatorname{right}(\mathbf{x})) & =w_{1}^{\prime}(\xi(i), \xi(\operatorname{right}(\mathbf{x})))=w_{1}^{\prime}(\xi(j), \xi(\operatorname{right}(\overline{\mathbf{x}}))) \\
& =w^{\prime}(j, \operatorname{right}(\overline{\mathbf{x}}))
\end{aligned}
$$

This completes the proof of the proposition.

Proposition 9.5 shows that the induction step is therefore also achieved in Case 5

Case 6. It remains to consider the case where all boundary equations of the form $(i, \mathrm{x}, j, \overline{\mathrm{x}})$ with $\operatorname{right}(\mathrm{x})=\max I$ are elastic (since Case 5 does not apply), and Case 4 does not apply. We set $r=\max I$ and we let $\mathcal{E}$ be the set of all elastic equations $(i, \mathrm{x}, j, \overline{\mathrm{x}}) \in \mathcal{B}$ such that $\operatorname{right}(\mathrm{x})=\operatorname{right}(\overline{\mathrm{x}})=r$.

We let $r^{-}$be the maximum among the elements $i \in I \backslash\{r\}$ which satisfy at least one of the following conditions:

- $i=\min I$

- there is no box of the form $(i, \mathrm{x})$;

- there is a boundary equation of the form $(i, \mathrm{x}, j, \overline{\mathrm{x}}) \in \mathcal{B} \backslash \mathcal{E}$;

- $i \in \operatorname{right}(\mathrm{X})$.

By applying repeatedly Proposition 9.2 of the auxiliary step, we may push forward the periods of the boundary equations in $\mathcal{E}$ until $r^{-}$is left behind so that we may assume that the following condition holds:

$$
\forall i \in I\left(r^{-} \leq i<r \Longleftrightarrow \exists \text { a box }(i, \mathrm{x}) \operatorname{such} \text { that } \operatorname{right}(\mathrm{x})=r\right) .
$$

We now set

$$
\ell=\max \{i \in I: \exists(i, \mathrm{x}, j, \overline{\mathrm{x}}) \in \mathcal{E}: i<j\} .
$$

In order to align with $\ell$ the left boundary $i$ of all equations $(i, \mathrm{x}, j, \overline{\mathrm{x}}), i<j$, of $\mathcal{E}$, we construct an intermediate pair $\left(\mathcal{S}_{0}, \mathcal{M}_{0}\right)$ as follows (see Figure 7 ). Let

$$
\begin{aligned}
\mathcal{S}_{0} & =\left(\mathrm{X}_{0}, I_{0}, \zeta_{0}, \chi_{0}, \text { right }_{0}, \mathcal{B}_{0}\right) \\
\mathcal{M}_{0} & =\left(w, \iota_{0}, \Phi_{0}\right) .
\end{aligned}
$$

Let $\mathcal{E}^{\prime}$ be the set consisting of all boundary equations $\left(i_{e}, \mathrm{x}, j_{e}, \overline{\mathrm{x}}\right) \in \mathcal{E}$ such that $i_{e}<\min \left\{j_{e}, \ell\right\}$.

- For each $e \in \mathcal{E}^{\prime}$, we introduce four new variables $\mathrm{y}_{e}, \overline{\mathrm{y}}_{e}, \mathrm{z}_{e}, \overline{\mathrm{z}}_{e}$ and we let

$$
\mathrm{X}_{0}=\mathrm{X} \uplus\left\{\mathrm{y}_{e}, \overline{\mathrm{y}}_{e}, \mathrm{z}_{e}, \overline{\mathrm{z}}_{e}: e \in \mathcal{E}^{\prime}\right\} .
$$




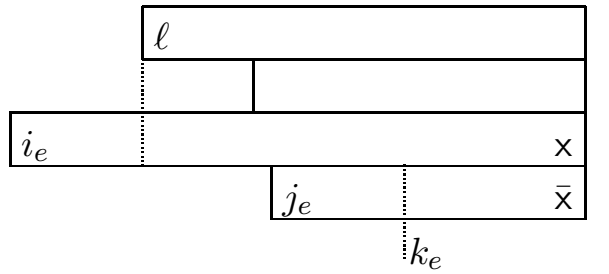

(a)

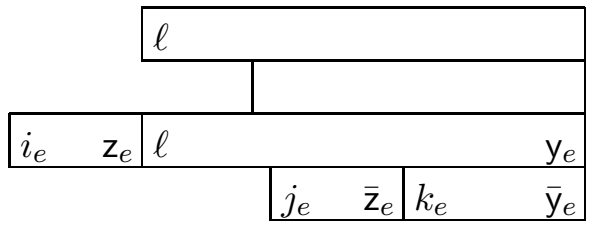

(b)

Figure 7: Aligning boxes to $\ell$ : before $(a)$ and after $(b)$.

- For each $e \in \mathcal{E}^{\prime}$, we let $\beta_{e} \in \alpha_{w}$ be such that $\mathrm{R}$ satisfies the pseudoidentity $w\left(i_{e}, \ell\right)=w\left[\iota\left(j_{e}\right), \beta_{e}[\right.$.

- By Proposition 8.1, there is a common refinement $\left(I_{0}, \iota_{0}, \Phi_{0}\right)$ of the following factorization schemes for $w:(I, \iota, \Phi)$ and $\left(\left\{\beta_{e}\right\},\left\{\beta_{e}\right\} \hookrightarrow \alpha_{w}+1, \emptyset\right)$, with $e \in \mathcal{E}^{\prime}$. Without loss of generality, we may assume that $I \subseteq I_{0}$ and $\iota=\left.\iota_{0}\right|_{I}$. We let $k_{e}=\iota_{0}^{-1}\left(\beta_{e}\right)$.

- For each $e \in \mathcal{E}^{\prime}$, we set $\operatorname{right}_{0}\left(\mathrm{y}_{e}\right)=\operatorname{right}_{0}\left(\overline{\mathrm{y}}_{e}\right)=r$, $\operatorname{right}_{0}\left(\mathrm{z}_{e}\right)=\ell$ and $\operatorname{right}_{0}\left(\bar{z}_{e}\right)=k_{e}$. We extend right ${ }_{0}$ to all of $\mathrm{X}_{0}$ so that it coincides with right on $\mathrm{X}$.

- The set $\mathcal{B}_{0}$ is obtained from $\mathcal{B}$ by replacing each $e \in \mathcal{E}^{\prime}$ and its dual by two new boundary equations, namely $\left(\ell, \mathrm{y}_{e}, k_{e}, \overline{\mathrm{y}}_{e}\right)$ and $\left(i_{e}, \mathrm{z}_{e}, j_{e}, \overline{\mathbf{z}}_{e}\right)$, together with their duals.

It is routine to establish the following result.

Proposition 9.6 The triple $\mathcal{M}_{0}$ is a model of the system of boundary equations $\mathcal{S}_{0}$ such that:

(1) if $\mathcal{S}_{0}$ admits a model in $\kappa$-terms, then so does $\mathcal{S}$;

(2) the set $\mathcal{E}_{0}^{\prime}$ corresponding to the system $\mathcal{S}_{0}$ is empty;

(3) $\left[\mathfrak{S}_{0}, \mathcal{M}_{0}\right]=[\mathcal{S}, \mathcal{M}]$.

Hence, we may assume that $\mathcal{E}^{\prime}=\emptyset$, which we do from hereon. This means that, for all boundary equations $(i, \mathrm{x}, j, \overline{\mathrm{x}}) \in \mathcal{B}$ such that $i<j$ and $\operatorname{right}(\mathrm{x})=$ $r$, the index $i$ is the same, namely what was above denoted by $\ell$. Let those elastic boundary equations be $\left(\ell, \mathrm{x}_{1}, j_{1}, \overline{\mathrm{x}}_{1}\right), \ldots,\left(\ell, \mathrm{x}_{n}, j_{n}, \overline{\mathrm{x}}_{n}\right)$, with $j_{1} \leq \cdots \leq$ $j_{n}$. Since $\mathcal{M}$ is a model of $\mathcal{S}$, it follows that

$$
\mathrm{R} \models w\left(\ell, j_{m}\right) w(\ell, r)=w\left(\ell, j_{m}\right) w\left(j_{m}, r\right)=w(\ell, r) \quad(m=1, \ldots, n) .
$$

Taking into account that Case 4 does not apply, so that we are assuming that $c\left(w\left(\ell, j_{m}\right)\right)=c(w(\ell, r))$ for $m=1, \ldots, n$, we obtain, using Lemma 4.3. the following equivalent condition:

$$
\mathrm{R} \models\left(w\left(\ell, j_{1}\right)\right)^{\omega}=\cdots=\left(w\left(\ell, j_{n}\right)\right)^{\omega}=w(\ell, r) .
$$


We will apply Proposition 5.5 to reduce the satisfaction of the elastic boundary equations in $\mathcal{E}$ expressed by condition (9.9) into another set of boundary equations which will guarantee (9.10). In the process, we will introduce perhaps very many boundary equations but we will reduce the induction parameter $[\mathcal{S}, \mathcal{M}]$, which is what we need to achieve the induction step. A little extra work will be required to handle the constraints, which will be done using the method that was introduced for the proof of Proposition 6.4. Informally, this is achieved by repeating, modulo R, the "longest" basis of the $\omega$-powers (as in (9.10) ), namely $w\left(\ell, j_{n}\right)$, sufficiently many times to guarantee that we enter into a cycle in terms of the constraints.

We start by introducing the reduced factorizations, modulo $\mathrm{R}$, given by Proposition [5.5] there are pseudowords $u, v_{1}, \ldots, v_{n}$ and positive exponents $e_{1}, \ldots, e_{n}$ such that for $m=1, \ldots, n$,

$$
\begin{aligned}
& \mathrm{R} \models w\left(\ell, j_{m}\right)=u^{e_{m}} v_{m}, \\
& \mathrm{R} \models v_{m} u=u
\end{aligned}
$$

where all the products, including $u u$, are reduced. Let $e=\max \left\{e_{m}: m=\right.$ $1, \ldots, n\}$. By Fact 5.3 , there are induced factorizations of the $w\left(\ell, j_{m}\right)$ which are described by appropriate choices of ordinals $\beta_{q}$ with $\iota(\ell)=\beta_{0}<\cdots<\beta_{e}$ such that

$$
\begin{aligned}
& w\left[\beta_{q-1}, \beta_{q}\left[= { } _ { \mathrm { R } } u \text { and } w \left[\beta_{e_{m}}, \iota\left(j_{m}\right)\left[={ }_{\mathrm{R}} v_{m}\right.\right.\right.\right. \\
& \quad(q=1, \ldots, e ; m=1, \ldots, n) .
\end{aligned}
$$

Observe that $\beta_{q}=\beta_{0}+\left(\beta_{1}-\beta_{0}\right) q$.

To handle the constraints, recall that a standard combinatorial argument shows that there exist integers $h_{S}$ and $n_{S}$ such that $1<h_{S}<n_{S}$ and, for all $s_{1}, \ldots, s_{n_{S}} \in S$,

$$
s_{1} \cdots s_{n_{S}}=s_{1} \cdots s_{h_{S}}\left(s_{1+h_{S}} \cdots s_{n_{S}}\right)^{\omega-1} .
$$

We now construct the new pair $\left(\mathcal{S}_{1}, \mathcal{M}_{1}\right)$, where

$$
\begin{aligned}
\mathcal{S}_{1} & =\left(\mathrm{X}_{1}, I_{1}, \zeta_{1}, \chi_{1}, \text { right }_{1}, \mathcal{B}_{1}\right) \\
\mathcal{M}_{1} & =\left(w, \iota_{1}, \Phi_{1}\right) .
\end{aligned}
$$

The various components are described as follows.

- The set $\mathrm{X}_{1}$ is obtained from $\mathrm{X}$ by adding new variables $\mathrm{y}_{q}, \overline{\mathrm{y}}_{q}, \mathrm{z}_{m}, \overline{\mathrm{z}}_{m}, \mathrm{t}_{i}, \overline{\mathrm{t}}_{i}$ with $q=1, \ldots, e, m=1, \ldots, n$ and $i=1, \ldots, n_{S}-1$.

- Let $J$ be the set consisting of the following ordinals:

$-\beta_{q}=\beta_{0}+\left(\beta_{1}-\beta_{0}\right) q, \quad(q=0, \ldots, e+1) ;$

$-\gamma_{m}=\beta_{0}+\iota\left(j_{m}\right)-\beta_{e_{m}}, \quad(m=1, \ldots, n) ;$

$-\delta_{p}=\beta_{0}+\left(\beta_{e}-\beta_{0}\right) p, \quad\left(p=0, \ldots, n_{S}\right)$. 
Note that in the first line, we define a new ordinal $\beta_{e+1}$. Since every finite power of $u$ is a prefix of $w(\ell, r)$, we have $w\left[\beta_{e}, \beta_{e+1}\left[=_{\mathrm{R}} u={ }_{\mathrm{R}} w\left[\beta_{q-1}, \beta_{q}[\right.\right.\right.$ for $q=1, \ldots, e$.

Also, by (9.12), R satisfies $u=v_{m} u$, and since the product $v_{m} u$ is reduced, we have $\alpha_{u}>\alpha_{v_{m}}$. Using (9.11), we obtain $\beta_{e} \leq \iota\left(j_{n}\right)<\beta_{e+1}$.

Notice finally that, by Proposition 5.5, the product $u u$ is reduced. Hence $\mathrm{R} \not \forall u^{2}=u$, and by (9.11), we obtain $\gamma_{m}<\beta_{1}$. Let $\left(I_{1}, \iota_{1}, \Phi_{1}\right)$ be a common refinement for the following two factorization schemes for $w$ : $(I, \iota, \Phi)$ and $\left(J, J \hookrightarrow \alpha_{w}+1, \emptyset\right)$. We let

(1) $b_{q}=\iota_{1}^{-1}\left(\beta_{q}\right) \quad$ (so that $b_{0}=\ell$ );

(2) $c_{m}=\iota_{1}^{-1}\left(\gamma_{m}\right)$;

(3) $d_{p}=\iota_{1}^{-1}\left(\delta_{p}\right) \quad$ (so that $d_{0}=\ell$ and $\left.d_{1}=b_{e}\right)$.

- For $i, j \in I_{1}$ with $i \prec j$, we set

$$
\begin{aligned}
\zeta_{1}(i, j) & =\left\{\varphi\left(\Phi_{1}(i, j, s)\right): \Phi_{1}(i, j, s) \text { is defined }\right\} \\
\chi_{1}(i, j) & =\vec{c}\left(w \left[\iota_{1}(i), \iota_{1}(j)[) .\right.\right.
\end{aligned}
$$

- Denote the composite $\iota_{1}^{-1} \circ \iota$ by $\xi$. We define

$$
\operatorname{right}_{1}(\mathrm{x})= \begin{cases}\xi(\operatorname{right}(\mathrm{x})) & \text { if } \mathrm{x} \in \mathrm{X} \\ b_{q} & \text { if } \mathrm{x}=\mathrm{y}_{q}, q \in\{1, \ldots, e\} \\ b_{q+1} & \text { if } \mathrm{x}=\overline{\mathrm{y}}_{q}, q \in\{1, \ldots, e\} \\ b_{1} & \text { if } \mathrm{x} \in\left\{\mathrm{z}_{m}, \overline{\mathrm{z}}_{m}\right\}, m \in\{1, \ldots, n\} \\ d_{p} & \text { if } \mathrm{x}=\mathrm{t}_{p}, p \in\left\{1, \ldots, n_{S}-1\right\} \\ d_{p+1} & \text { if } \mathrm{x}=\overline{\mathrm{t}}_{p}, p \in\left\{1, \ldots, n_{S}-1\right\} .\end{cases}
$$

- Finally, the set $\mathcal{B}_{1}$ of boundary equations consists of all the following 4-tuples:

$-(\xi(i), \mathrm{x}, \xi(j), \overline{\mathrm{x}})$ if $(i, \mathrm{x}, j, \overline{\mathrm{x}}) \in \mathcal{B} \backslash \mathcal{E}$;

- $\left(b_{q-1}, \mathrm{y}_{q}, b_{q}, \overline{\mathrm{y}}_{q}\right)$, together with its dual $(q=1, \ldots, e)$;

- $\left(\ell, \mathbf{z}_{m}, c_{m}, \overline{\mathbf{z}}_{m}\right)$, together with its dual $(m=1, \ldots, n)$;

- $\left(d_{p-1}, \mathrm{t}_{p}, d_{p}, \overline{\mathrm{t}}_{p}\right)$, together with its dual $\left(p=1, \ldots, n_{S}-1\right)$.

Let us illustrate the new boundary equations with an example (see Figure 8 , where the dashes emphasize that, over R, every finite power of $u$ is a prefix of $w(\ell, r))$. Assume that $n=2$, that is, we start with two elastic boundary equations $\left(\ell, \mathrm{x}_{1}, j_{1}, \overline{\mathrm{x}}_{1}\right)$, and $\left(\ell, \mathrm{x}_{2}, j_{2}, \overline{\mathrm{x}}_{2}\right)$. Boundary equations involving $\mathrm{y}_{i}$, $\overline{\mathrm{y}}_{i}, \mathrm{z}_{i}$, and $\overline{\mathrm{z}}_{i}$ are meant to handle the periodicity over $\mathrm{R}$ of $w(\ell, r)$. The first group of boundary equations, involving variables $\mathrm{y}_{i}, \overline{\mathrm{y}}_{i}$, takes care of the fact that, by (9.13), we have $w\left[\beta_{q-1}, \beta_{q}\left[={ }_{\mathrm{R}} w\left[\beta_{q}, \beta_{q+1}\left[=_{\mathrm{R}} u\right.\right.\right.\right.$. The purpose of the second group is to encode that $w\left[\beta_{e_{m}}, \iota\left(j_{m}\right)\left[={ }_{\mathrm{R}} v_{m}\right.\right.$. Finally, assume that $n_{S}=4$. The last boundary equations are added to take care of the constraints on the finite semigroup $S$. 

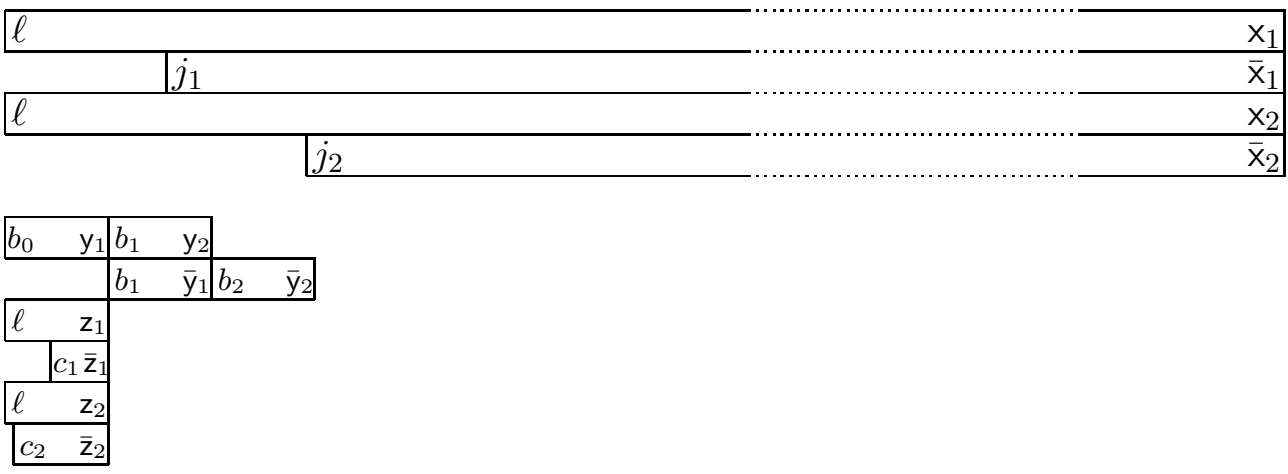

\begin{tabular}{|ll|ll|ll|ll|}
\hline$d_{0}$ & $\mathrm{t}_{1}$ & $d_{1}$ & $\mathrm{t}_{2}$ & $d_{2}$ & $\mathrm{t}_{3}$ & \\
\hline & & $d_{1}$ & $\overline{\mathrm{t}}_{1}$ & $d_{2}$ & $\overline{\mathrm{t}}_{2}$ & $d_{3}$ & $\overline{\mathrm{t}}_{3}$ \\
\cline { 2 - 6 }
\end{tabular}

Figure 8: Handling constraints in $S$ with new boundary equations

From the construction, it is immediate that $\mathcal{M}_{1}$ is a model of the system $\mathcal{S}_{1}$ of boundary equations and that the inequality $\left[\mathcal{S}_{1}, \mathcal{M}_{1}\right]<[\mathcal{S}, \mathcal{M}]$ holds since we have kept the ordinal which determines the first component of the induction parameter while reducing the second component to zero. To complete the proof of the induction step also in the present case, it remains to show that Property (P.2) holds.

Proposition 9.7 Suppose that $\mathcal{M}_{1}^{\prime}=\left(w_{1}^{\prime}, \iota_{1}^{\prime}, \Phi_{1}^{\prime}\right)$ is a model of $\mathcal{S}_{1}$ in $\kappa$ terms. Then $\mathcal{S}$ also admits a model in $\kappa$-terms.

Proof. Set $\tau=\iota_{1}^{\prime} \circ \iota_{1}^{-1}$. We let $\mathcal{M}^{\prime}=\left(w^{\prime}, \iota^{\prime}, \Phi^{\prime}\right)$ be defined as follows:

- the pseudoword $w^{\prime}$ is given by the $\kappa$-term

$$
w^{\prime}=w_{1}^{\prime}\left[0, \tau\left(\delta_{h_{S}}\right)\left[\cdot \left(w _ { 1 } ^ { \prime } \left[\tau\left(\delta_{h_{S}}\right), \tau\left(\delta_{n_{S}}\right)[)^{\omega-1} \cdot w_{1}^{\prime}\left[\tau\left(\delta_{n_{S}}\right), \tau(\iota(r))[;\right.\right.\right.\right.\right.
$$

- let $K=I \uplus\left\{r_{h_{S}}, r_{n_{S}}\right\}$ be ordered so that the function $\iota_{(0)}^{\prime}: K \rightarrow \alpha_{w_{1}^{\prime}}+1$ is order preserving, where $\iota_{(0)}^{\prime}$ is defined to be the extension of $\iota_{1}^{\prime} \circ \xi$ that is determined by $\iota_{(0)}^{\prime}\left(r_{h_{S}}\right)=\tau\left(\delta_{h_{S}}\right)$ and $\iota_{(0)}^{\prime}\left(r_{n_{S}}\right)=\tau\left(\delta_{n_{S}}\right)$;

- let $\left(K, \iota_{(0)}^{\prime}, \Phi_{(0)}^{\prime}\right)$ be the factorization scheme of $w^{\prime}$ which is obtained from $\left(I_{1}, \iota_{1}^{\prime}, \Phi_{1}^{\prime}\right)$ by restriction;

- for $i \in I$, set

$$
\iota^{\prime}(i)= \begin{cases}\left(\iota_{1}^{\prime} \circ \xi\right)(i) & \text { if } i<r \\ \alpha_{w^{\prime}} & \text { if } i=r\end{cases}
$$

- for $i, j \in I$ with $i \prec j$ and $s \in \zeta(i, j)$, we let $\Phi^{\prime}(i, j, s)=\Phi_{(0)}^{\prime}(i, j, s)$ if $j<r$; in the case where $j=r$, so that $i=j_{n}$ (recall that $j_{n}=\max \left\{j_{m}\right.$ : 
$m=1, \ldots, n\})$, we set

$$
\Phi^{\prime}\left(j_{n}, r, s\right)=\Phi_{(0)}^{\prime}\left(j_{n}, r_{h_{S}}, s_{1}\right) \cdot\left(\Phi_{(0)}^{\prime}\left(r_{h_{S}}, r_{n_{S}}, s_{2}\right)\right)^{\omega-1} \cdot \Phi_{(0)}^{\prime}\left(r_{n_{S}}, r, s_{3}\right),
$$

where $s=s_{1} s_{2} s_{3}$ is chosen to be a factorization such that $\Phi_{(0)}^{\prime}\left(j_{n}, r_{h_{S}}, s_{1}\right)$, $\Phi_{(0)}^{\prime}\left(r_{h_{S}}, r_{n_{S}}, s_{2}\right)$, and $\Phi_{(0)}^{\prime}\left(r_{n_{S}}, r, s_{3}\right)$ are defined. Note that there exists at least one such factorization: since $\left(I_{1}, \iota_{1}, \Phi_{1}\right)$ is a refinement of the factorization scheme $(I, \iota, \Phi)$ for $w$, and

$$
\xi\left(j_{n}\right) \prec d_{2} \prec \cdots \prec d_{n_{S}} \prec \xi(r)
$$

in $I_{1}$, there exists a factorization $s=s_{1}^{\prime} s_{2}^{\prime} \cdots s_{n_{S}}^{\prime}$ such that we have $s_{1}^{\prime} \in$ $\zeta_{1}\left(\xi\left(j_{n}\right), d_{2}\right), s_{i}^{\prime} \in \zeta_{1}\left(d_{i}, d_{i+1}\right)\left(i=2, \ldots, n_{S}-1\right)$, and $s_{n_{S}}^{\prime} \in \zeta_{1}\left(d_{n_{S}}, \xi(r)\right)$, so that we may take $s_{1}=s_{1}^{\prime} \cdots s_{h_{S}-1}^{\prime}, s_{2}=s_{h_{S}}^{\prime} \cdots s_{n_{S}-1}^{\prime}$, and $s_{3}=s_{n_{S}}^{\prime}$.

The mappings are shown on Figure 9 Note that not all triangles in this diagram commute, and that $\tau$ is a partial function.

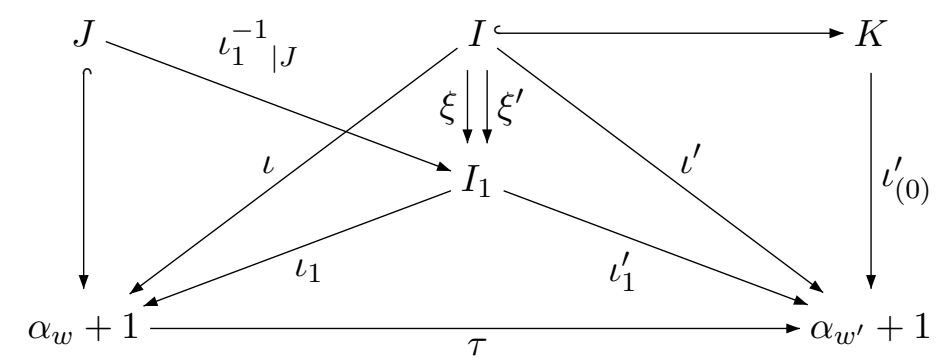

Figure 9: Involved mappings

We claim that $\mathcal{M}^{\prime}$ is a model of $\mathcal{S}$ in $\kappa$-terms. Let $i, j \in I$ be such that $i \prec j$. We first consider the case where $j<r$. Then we have the following equalities, where, if appropriate, we assume that $s \in \zeta(i, j)$ :

$$
\begin{aligned}
\varphi\left(\Phi^{\prime}(i, j, s)\right) & =\varphi\left(\Phi_{(0)}^{\prime}(i, j, s)\right)=s \\
\vec{c}\left(w^{\prime}(i, j)\right) & =\vec{c}\left(w_{1}^{\prime}\left(\iota_{1}^{\prime-1}\left(\iota^{\prime}(i)\right), \iota_{1}^{\prime-1}\left(\iota^{\prime}(j)\right)\right)\right) \\
& =\chi_{1}\left(t, \iota_{1}^{\prime-1}\left(\iota^{\prime}(j)\right)\right) \quad \text { if } t \prec \iota_{1}^{\prime-1}\left(\iota^{\prime}(j)\right) \\
& =\vec{c}\left(w\left(\iota^{-1}\left(\iota_{1}(t)\right), j\right)\right) \\
& =\vec{c}(w(i, j))=\chi(i, j) \\
\mathrm{R} \models \Phi^{\prime}(i, j, s) & =\Phi_{(0)}^{\prime}(i, j, s)=w^{\prime}(i, j)
\end{aligned}
$$

where the validity of the last pseudoidentity over $\mathrm{R}$ follows from the definition of factorization scheme. Assume next that $j=r$, so that $i=j_{n}$. For 
$s \in \zeta(i, j)$, from the definition of $\Phi^{\prime}\left(j_{n}, r, s\right)$ in 9.15) and the hypothesis that (9.14) holds for all elements of $S$, we deduce that

$$
\begin{aligned}
\varphi\left(\Phi^{\prime}\left(j_{n}, r, s\right)\right) & =\varphi\left(\Phi_{(0)}^{\prime}\left(j_{n}, r_{h_{S}}, s_{1}\right)\right) \cdot \varphi\left(\Phi_{(0)}^{\prime}\left(r_{h_{S}}, r_{n_{S}}, s_{2}\right)\right) \cdot \varphi\left(\Phi_{(0)}^{\prime}\left(r_{n_{S}}, r, s_{3}\right)\right) \\
& =s_{1} s_{2} s_{3}=s
\end{aligned}
$$

for an appropriate choice of factorization $s=s_{1} s_{2} s_{3}$. This completes the proof of (M.1) For (M.2) we perform the following calculations:

$$
\begin{aligned}
\vec{c}\left(w^{\prime}\left(j_{n}, r\right)\right) & =\vec{c}\left(w_{1}^{\prime}\left(\iota_{1}^{\prime-1}\left(\iota^{\prime}\left(j_{n}\right)\right), \iota_{1}^{\prime-1}\left(\iota^{\prime}(r)\right)\right)\right) \\
& =\chi_{1}\left(\iota_{1}^{-1}\left(\iota^{\prime}\left(j_{n}\right)\right), \iota_{1}^{\prime-1}\left(\iota^{\prime}(r)\right)\right) \\
& =\vec{c}\left(w \left[\delta_{n_{S}}, \iota(r)[)=\vec{c}\left(w \left[\iota\left(j_{n}\right), \iota(r)[)\right.\right.\right.\right. \\
& =\vec{c}\left(w\left(j_{n}, r\right)\right)=\chi\left(j_{n}, r\right) .
\end{aligned}
$$

In turn, the following calculations yield (M.3) for $s \in \zeta\left(j_{n}, r\right)$ and a suitable choice of factorization $s=s_{1} s_{2} s_{3}$ :

$$
\begin{aligned}
\mathrm{R} \models \Phi^{\prime}\left(j_{n}, r, s\right) & =\Phi_{(0)}^{\prime}\left(j_{n}, r_{h_{S}}, s_{1}\right) \cdot\left(\Phi_{(0)}^{\prime}\left(r_{h_{S}}, r_{n_{S}}, s_{2}\right)\right)^{\omega-1} \cdot \Phi_{(0)}^{\prime}\left(r_{n_{S}}, r, s_{3}\right) \\
& =w^{\prime}\left(j_{n}, r_{h_{S}}\right) \cdot\left(w^{\prime}\left(r_{h_{S}}, r_{n_{S}}\right)\right)^{\omega-1} \cdot w^{\prime}\left(r_{n_{S}}, r\right) \\
& =w^{\prime}\left(j_{n}, r\right) .
\end{aligned}
$$

Finally, for condition (M.4) let $(i, x, j, \bar{x})$ be a boundary equation in $\mathcal{B}$. If it does not belong to $\mathcal{E}$ then, by the choice of $\mathcal{B}_{1},(\xi(i), \times, \xi(j), \bar{x})$ belongs to $\mathcal{B}_{1}$ and so, since $\mathcal{M}_{1}^{\prime}$ is a model of $\mathcal{S}_{1}$, R satisfies the following pseudoidentities:

$$
\begin{aligned}
w^{\prime}(i, \operatorname{right}(\mathbf{x})) & \left.=w_{1}^{\prime}(\xi(i), \xi(\operatorname{right}(\mathbf{x})))=w_{1}^{\prime}\left(\xi(i), \operatorname{right}_{1}(\mathbf{x})\right)\right) \\
& \left.=w_{1}^{\prime}\left(\xi(j), \operatorname{right}_{1}(\overline{\mathbf{x}})\right)\right)=w_{1}^{\prime}(\xi(j), \xi(\operatorname{right}(\overline{\mathrm{x}}))) \\
& =w^{\prime}(j, \operatorname{right}(\overline{\mathbf{x}})) .
\end{aligned}
$$

For the remaining boundary equations, it suffices to check that $\mathrm{R}$ satisfies each of the pseudoidentities $w^{\prime}(\ell, r)=w^{\prime}\left(j_{m}, r\right)(m=1, \ldots, n)$. Now, since $\mathcal{M}_{1}^{\prime}$ is a model of $\mathcal{S}_{1}$, R satisfies

$$
\begin{aligned}
w^{\prime}\left[\beta_{q-1}, \beta_{q}[\right. & =w_{1}^{\prime}\left[\beta_{q-1}, \beta_{q}\left[=w_{1}^{\prime}\left(\iota_{1}^{\prime-1}\left(\beta_{q-1}\right), \operatorname{right}_{1}\left(\mathrm{y}_{q}\right)\right)\right.\right. \\
& =w_{1}^{\prime}\left(\iota_{1}^{\prime}-1\left(\beta_{q}\right), \operatorname{right}_{1}\left(\overline{\mathrm{y}}_{q}\right)\right)=w_{1}^{\prime}\left[\beta_{q}, \beta_{q+1}[\right. \\
& =w^{\prime}\left[\beta_{q}, \beta_{q+1}[\quad(q=1, \ldots, e)\right. \\
& =w^{\prime}\left[\beta_{0}, \beta_{1}[\right.
\end{aligned}
$$

and, similarly, R satisfies

$$
\begin{aligned}
w^{\prime}\left[\beta_{e_{m}}, \iota\left(j_{m}\right)\left[\cdot w ^ { \prime } \left[\beta_{0}, \beta_{1}[\right.\right.\right. & =w^{\prime}\left[\beta_{0}, \beta_{1}[\quad(m=1, \ldots, n), \text { and }\right. \\
w^{\prime}\left[\delta_{i-1}, \delta_{i}[\right. & =w^{\prime}\left[\delta_{i}, \delta_{i+1}\left[\quad\left(i=1, \ldots, n_{S}-1\right)\right.\right. \\
& =\left(w ^ { \prime } \left[\beta_{0}, \beta_{1}[)^{e} .\right.\right.
\end{aligned}
$$


Hence R also satisfies

$$
\begin{aligned}
w^{\prime}(\ell, r)= & w^{\prime}\left(\ell, j_{m}\right) \cdot w^{\prime}\left(j_{m}, r\right) \\
= & \left(w ^ { \prime } \left[\beta_{0}, \beta_{1}[)^{e_{m}} w^{\prime}\left[\beta_{e_{m}}, \iota\left(j_{m}\right)[\right.\right.\right. \\
& \cdot\left(w ^ { \prime } \left[\beta_{0}, \beta_{1}[)^{e-e_{m}+\left(h_{S}-2\right) e} \cdot\left(w ^ { \prime } \left[\beta_{0}, \beta_{1}[)^{\omega-1} \cdot w^{\prime}\left[\delta_{n_{S}}, \iota(r)[\right.\right.\right.\right.\right. \\
= & \left(w ^ { \prime } \left[\beta_{0}, \beta_{1}[)^{\left(h_{S}-1\right) e} \cdot\left(w ^ { \prime } \left[\beta_{0}, \beta_{1}[)^{\omega-1} \cdot w^{\prime}\left[\delta_{n_{S}}, \iota(r)[\right.\right.\right.\right.\right. \\
= & w^{\prime}\left(j_{m}, r\right) .
\end{aligned}
$$

This concludes the proof of the proposition.

We have thus concluded all the cases of the induction step and we have therefore proved the following main result.

Theorem 9.8 The pseudovariety $\mathrm{R}$ is completely $\kappa$-reducible.

\section{Acknowledgments}

The authors benefited from the presentations of Makanin's algorithm in 35. Chapter 12] and in Ana Moura's unpublished Master's dissertation [39.

The work of the authors was supported, in part, by the PESSOA bilateral project Egide/Grices 11113YM Automata, profinite semigroups and symbolic dynamics.

The work of the first author was supported, in part, by Fundação para a Ciência e a Tecnologia (FCT) through the Centro de Matemática da Universidade do Porto. It was done in part while the author was visiting the LIAFA at the University Denis Diderot (Paris 7) and CNRS, whose hospitality is gratefully acknowledged, benefiting from a sabbatical fellowship from FCT.

The work of the second author was also supported, in part, by FCT through the Centro de Matemática da Universidade do Minho. It was done in part while the author visited the LIAFA at the University Denis Diderot (Paris 7) and CNRS, whose hospitality is gratefully acknowledged.

\section{References}

[1] D. Albert, R. Baldinger, and J. Rhodes, The identity problem for finite semigroups (the undecidability of), J. Symbolic Logic 57 (1992), 179192.

[2] J. Almeida, Finite Semigroups and Universal Algebra, World Scientific, Singapore, 1995, English translation.

[3] _ Hyperdecidable pseudovarieties and the calculation of semidirect products, 9 (1999), 241-261. 
[4] _ On a problem of Brzozowski and Fich, Semigroups and Applications (Singapore) (J. M. Howie and N. Ruskuc, eds.), World Scientific, 1999, pp. 1-17.

[5] _ Dynamics of implicit operations and tameness of pseudovarieties of groups, Trans. Amer. Math. Soc. 354 (2002), 387-411.

[6] _ Finite semigroups: an introduction to a unified theory of pseudovarieties, Semigroups, Algorithms, Automata and Languages (Singapore) (G. M. S. Gomes, J.-É. Pin, and P. V. Silva, eds.), World Scientific, 2002, pp. 3-64.

[7] _ Profinite structures and dynamics, CIM Bulletin 14 (2003), $8-18$.

[8] _ Profinite semigroups and applications, Structural Theory of Automata, Semigroups, and Universal Algebra (New York) (Valery B. Kudryavtsev and Ivo G. Rosenberg, eds.), NATO Science Series II: Mathematics, Physics and Chemistry, vol. 207, Springer, 2005, Proceedings of the NATO Advanced Study Institute on Structural Theory of Automata, Semigroups and Universal Algebra, Montréal, Québec, Canada, 7-18 July 2003.

[9] J. Almeida and A. Azevedo, The join of the pseudovarieties of $\mathcal{R}$-trivial and L-trivial monoids, J. Pure Appl. Algebra 60 (1989), 129-137.

[10] J. Almeida, J. C. Costa, and M. Zeitoun, Complete reducibility of pseudovarieties, To appear in the Proceedings of the Conference on Semigroups and Languages in honour of D. McAlister, Lisbon, July, 2005.

[11] J. Almeida, J. C. Costa, and M. Zeitoun, Tameness of pseudovariety joins involving R, Monatsh. Math. 146 (2005), no. 2, 89-111.

[12] J. Almeida and M. Delgado, Sur certains systèmes d'équations avec contraintes dans un groupe libre, Portugal. Math. 56 (1999), 409-417.

[13] _ Sur certains systèmes d'équations avec contraintes dans un groupe libre-addenda, Portugal. Math. 58 (2001), 379-387.

[14] _ Tameness of the pseudovariety of Abelian groups, 15 (2005), $327-338$.

[15] J. Almeida and B. Steinberg, On the decidability of iterated semidirect products and applications to complexity, 80 (2000), 50-74.

[16] _ Syntactic and global semigroup theory, a synthesis approach, Algorithmic Problems in Groups and Semigroups (J. C. Birget, S. W. Margolis, J. Meakin, and M. V. Sapir, eds.), Birkhäuser, 2000, pp. 1-23. 
[17] J. Almeida and P. G. Trotter, Hyperdecidability of pseudovarieties of orthogroups, Glasgow Math. J. 43 (2001), 67-83.

[18] J. Almeida and P. Weil, Relatively free profinite monoids: an introduction and examples, Semigroups, Formal Languages and Groups (Dordrecht) (J. B. Fountain, ed.), vol. 466, Kluwer Academic Publ., 1995, pp. $73-117$.

[19] _ Free profinite $\mathcal{R}$-trivial monoids, 7 (1997), 625-671.

[20] _ Profinite categories and semidirect products, J. Pure Appl. Algebra 123 (1998), 1-50.

[21] J. Almeida and M. Zeitoun, An automata-theoretic approach to the word problem for $\omega$-terms over $R$, Theoret. Comput. Sci. 370 (2007), no. 1-3, 131-169.

[22] C. J. Ash, Inevitable graphs: a proof of the type II conjecture and some related decision procedures, 1 (1991), 127-146.

[23] K. Auinger and B. Steinberg, On the extension problem for partial permutations, Proc. Amer. Math. Soc. 131 (2003), 2693-2703.

[24] _ The geometry of profinite graphs with applications to free groups and finite monoids, Trans. Amer. Math. Soc. 356 (2004), 805851.

[25] G. Baumslag, Residual nilpotence and relations in free groups, J. Algebra 2 (1965), 271-282.

[26] J.-C. Birget, Iteration of expansions - unambiguous semigroups, J. Pure Appl. Algebra 34 (1984), 1-55.

[27] S. Burris and H. P. Sankappanavar, A Course in Universal Algebra, Grad. Texts in Math., no. 78, Springer, Berlin, 1981.

[28] J. C. Costa and M. L. Teixeira, Tameness of the pseudovariety LSl, 14 (2004), 627-654.

[29] Th. Coulbois and A. Khélif, Equations in free groups are not finitely approximable, Proc. Amer. Math. Soc. 127 (1999), 963-965.

[30] S. Eilenberg, Automata, Languages and Machines, vol. A, Academic Press, New York, 1974.

[31] _ Automata, Languages and Machines, vol. B, Academic Press, New York, 1976.

[32] K. Henckell, S. Margolis, J.-É. Pin, and J. Rhodes, Ash's type II theorem, profinite topology and Malcev products. Part I, 1 (1991), 411-436. 
[33] B. Herwig and D. Lascar, Extending partial automorphisms and the profinite topology on free groups, Trans. Amer. Math. Soc. 352 (2000), 1985-2021.

[34] R. P. Hunter, Certain finitely generated compact zero-dimensional semigroups, J. Austral. Math. Soc., Ser. A 44 (1988), 265-270.

[35] M. Lothaire, Algebraic Combinatorics on Words, Cambridge University Press, Cambridge, UK, 2002.

[36] G. S. Makanin, The problem of solvability of equations in a free semigroup, Mat. Sb. (N.S.) 103 (2) (1977), 147-236, In Russian. English translation in: Math. USSR-Sb. 32 (1977) 128-198.

[37] _ Equations in a free semigroup, Amer. Math. Soc. Transl. (II Ser.) 117 (1981), 1-6.

[38] S. Margolis, M. Sapir, and P. Weil, Closed subgroups in pro- $V$ topologies and the extension problem for inverse automata, 11 (2001), 405-445.

[39] Ana I. P. Moura, Equations in the free monoid and in the free group, Master's thesis, Univ. Porto, December 2003.

[40] D. Perrin, Finite automata, Handbook of Theoretical Computer Science (Jan van Leeuwen, ed.), vol. B: Formal Models and Semantics, Elsevier, 1990, pp. 1-57.

[41] J.-É. Pin, Syntactic semigroups, Handbook of Formal Languages (G. Rozenberg and A. Salomaa, eds.), vol. 1, Springer, 1997, pp. 679746 .

[42] J.-É. Pin and P. Weil, Profinite semigroups, Mal'cev products and identities, J. Algebra 182 (1996), 604-626.

[43] Jean-Éric Pin, Varieties of formal languages, Plenum, 1986.

[44] W. Plandowski, Satisfiability of word equations is in NEXPTIME, Proceedings of the Symposium on the Theory of Computing (STOC'99), ACM, 1999, pp. 721-725.

[45] Satisfiability of word equations with constants is in PSPACE, Journal of the ACM 51 (2004), no. 3, 483-496.

[46] J. Reiterman, The Birkhoff theorem for finite algebras, Algebra Universalis 14 (1982), no. 1, 1-10.

[47] J. Rhodes, Undecidability, automata and pseudovarieties of finite semigroups, 9 (1999), 455-473. 
[48] J. Rhodes and B. Steinberg, Pointlike sets, hyperdecidability and the identity problem for finite semigroups, 9 (1999), 475-481.

[49] —, The q-Theory of Finite Semigroups, 2001-2004, Book under preparation. Preliminary versions available through http://mathstat.math.carleton.ca/ bsteinbg/qtheor.html.

[50] L. Ribes and P. A. Zalesskiı̌, The pro-p topology of a free group and algorithmic problems in semigroups, 4 (1994), 359-374.

[51] B. Steinberg, Inevitable graphs and profinite topologies: some solutions to algorithmic problems in monoid and automata theory, stemming from group theory, 11 (2001), 25-71.

[52] W. Thomas, Languages, automata, and logic, Handbook of Formal Languages. Beyond Words (G. Rozenberg and A. Salomaa, eds.), vol. 3, Springer, Berlin, 1997, pp. 389-455.

[53] P. Weil, Profinite methods in semigroup theory, 12 (2002), 137-178. 